Supporting Information for

\title{
Investigating the Photochemistry of C7 and C8 Functionalized N(5)-Ethyl- Flavinium Cation: A Computational Study
}

\author{
Brian D. Etz, Julie M. DuClos, Shubham Vyas* \\ Department of Chemistry, Colorado School of Mines, Golden CO 80401, United States \\ *Corresponding author: phone +1-303-273-3632, email svyas@ mines.edu
}

Table S1. Comparison of calculated Frank-Condon UV-Vis signatures of $\mathrm{Et}_{-} \mathrm{Fl}^{+}$using $\mathrm{M} 062 \mathrm{X} / 6-31+\mathrm{G}(\mathrm{d}, \mathrm{p})$ and $\mathrm{B} 3 \mathrm{LYP} / 6-31+\mathrm{G}(\mathrm{d}, \mathrm{p})$ level of theories with previously reported theoretical $^{*}$ and experimental* absorption wavelengths. Wavelengths are presented in $\mathrm{nm}$ and inside the brackets are the corresponding oscillator strengths.

\begin{tabular}{|c|c|c|c|c|}
\hline $\begin{array}{c}\text { FC } \\
\text { States }\end{array}$ & M062X/6-31+g(d,p) & B3LYP/6-31+g(d,p) & B3LYP/6-31G(d)* & Expt.* \\
\hline$S_{1}$ & $474[0.2495]$ & $563[0.1375]$ & $563[0.1363]$ & 557 \\
\hline$S_{2}$ & $359[0.2500]$ & $447[0.0192]$ & $447[0.0192]$ & N/A \\
\hline$S_{3}$ & $335[0.0023]$ & $422[0.2443]$ & $422[0.2433]$ & 414 \\
\hline
\end{tabular}

*Sichula, V.; Kucheryavy, P.; Khatmullin, R.; Hu, Y.; Mirzakulova, E.; Vyas, S.; Manzer, S. F.; Hadad, C. M.; Glusac, K. D. Electronic Properties of N(5)-Ethyl Flavinium Ion. J. Phys. Chem. A 2010, 114 (46), 12138-12147. 
Table S2. Dihedral angle for all the Et-Fl ${ }^{+}$derivatives optimized at the ground state at the B3LYP/6-31+G(d,p) level of theory using implicit solvation of acetonitrile. Dihedral angle (in degree) corresponds to C4 carbonyl oxygen to N5 position (O4-C4-C4 $\alpha$-N5). See Scheme 1 in main text for nomenclature.

\begin{tabular}{llll}
\hline Compound & $\begin{array}{l}\text { Dihedral } \\
\text { Angle }\end{array}$ & Compound & $\begin{array}{l}\text { Dihedral } \\
\text { Angle }\end{array}$ \\
\hline $\mathrm{Et}^{-} \mathrm{Fl}^{+}$ & -10 & $\mathrm{Et}-\mathrm{Fl}^{+}$ & -10 \\
$1 \mathrm{AB}$ & -13 & $6 \mathrm{AB}$ & -6 \\
$1 \mathrm{~A}$ & -13 & $6 \mathrm{~A}$ & -8 \\
$1 \mathrm{~B}$ & -12 & $6 \mathrm{~B}$ & -8 \\
$2 \mathrm{AB}$ & -13 & $7 \mathrm{AB}$ & -8 \\
2A & -13 & $7 \mathrm{~A}$ & -9 \\
$2 \mathrm{~B}$ & -12 & $7 \mathrm{~B}$ & -9 \\
$3 \mathrm{AB}$ & -13 & $8 \mathrm{AB}$ & -10 \\
$3 \mathrm{~A}$ & -13 & $8 \mathrm{~A}$ & -10 \\
$3 \mathrm{~B}$ & -12 & $8 \mathrm{~B}$ & -10 \\
$4 \mathrm{AB}$ & -11 & $9 \mathrm{AB}$ & -11 \\
$4 \mathrm{~A}$ & -11 & $9 \mathrm{~A}$ & -11 \\
$4 \mathrm{~B}$ & -11 & $9 \mathrm{~B}$ & -11 \\
$5 \mathrm{AB}$ & -12 & & \\
$5 \mathrm{~A}$ & -11 & & \\
$5 \mathrm{~B}$ & -11 & & \\
\hline
\end{tabular}



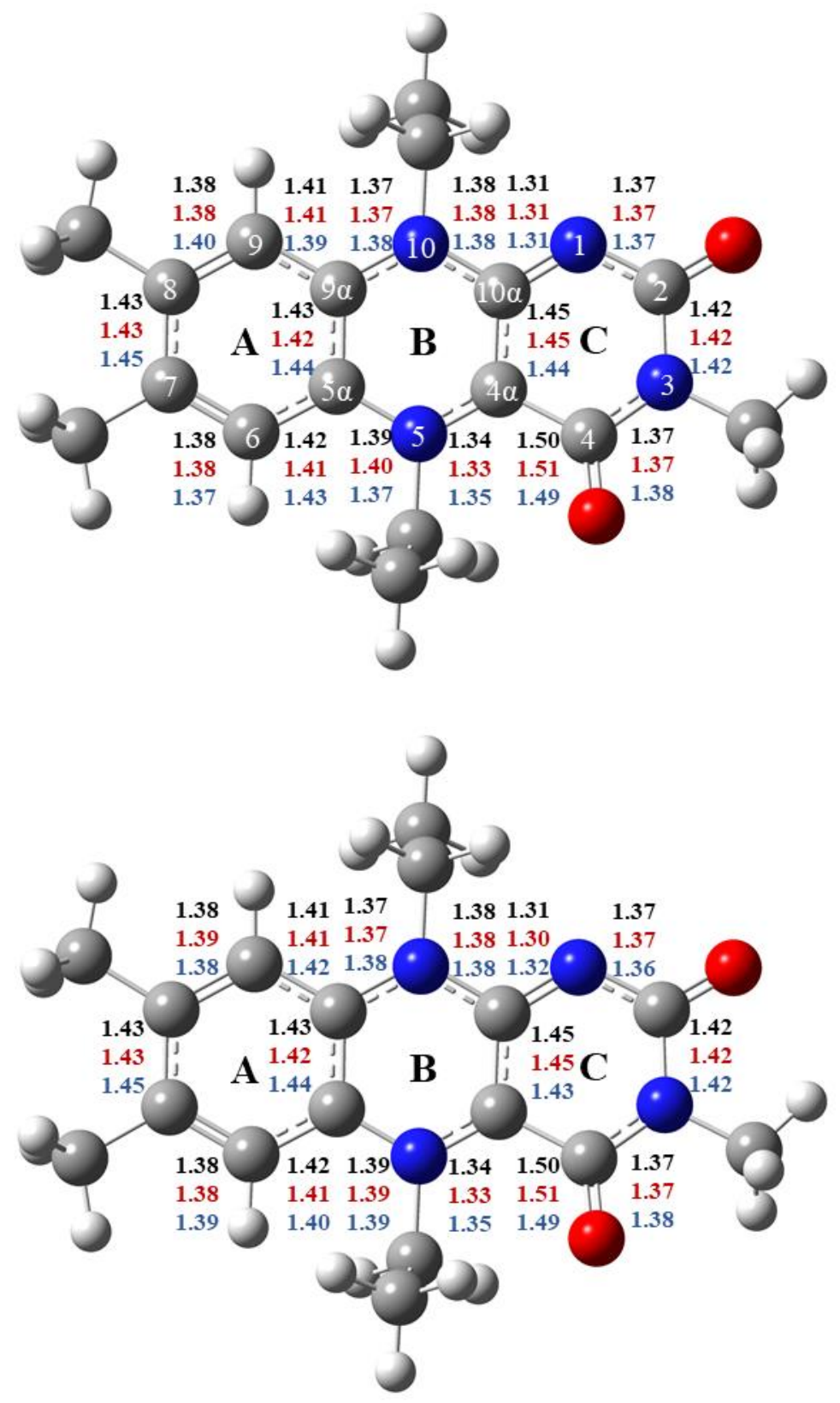

Figure S1. Bond lengths (in $\AA$ ) for $\mathrm{Et}^{-\mathrm{Fl}^{+}}$(black), 1A (top, red), 1B (bottom, red), 6A (top, blue), and 6B (bottom, blue) as calculated at B3LYP/6-31+G(d,p) level of theory using implicit solvation of acetonitrile. 
Table S3. The dominant character for $S_{1}$ and $S_{2}$ excited states for all derivatives as obtained by the calculations at TD-B3LYP/6-31+G(d,p) level of theory with implicit solvation of acetonitrile.

\begin{tabular}{|c|c|c|c|c|}
\hline Compound & Orbital Transition & $\lambda(\mathbf{n m})$ & $f$ & Excited State \\
\hline \multirow[t]{2}{*}{$\mathbf{E t}-\mathbf{F l}{ }^{+}$} & HOMO to LUMO & 563 & 0.1375 & 1 \\
\hline & HOMO-2 to LUMO & 447 & 0.0192 & 2 \\
\hline \multirow{2}{*}{$\begin{array}{c}1 \mathrm{AB} \\
{\left[\mathrm{CF}_{3}\right]}\end{array}$} & HOMO to LUMO & 528 & 0.1441 & 1 \\
\hline & HOMO-1 to LUMO & 473 & 0.0010 & 2 \\
\hline \multirow{2}{*}{$\begin{array}{c}1 \mathrm{~A} \\
{\left[\mathrm{CF}_{3}\right]}\end{array}$} & HOMO to LUMO & 569 & 0.1430 & 1 \\
\hline & HOMO-1 to LUMO & 463 & 0.0022 & 2 \\
\hline \multirow{2}{*}{$\begin{array}{c}1 \mathrm{~B} \\
{\left[\mathrm{CF}_{3}\right]}\end{array}$} & HOMO to LUMO & 525 & 0.1353 & 1 \\
\hline & HOMO-2 to LUMO & 457 & 0.0054 & 2 \\
\hline \multirow{2}{*}{$\begin{array}{c}2 \mathrm{AB} \\
{\left[\mathrm{NO}_{2}\right]}\end{array}$} & HOMO to LUMO & 521 & 0.1662 & 1 \\
\hline & HOMO-1 to LUMO & 482 & 0.0006 & 2 \\
\hline \multirow{2}{*}{$\begin{array}{c}2 \mathrm{~A} \\
{\left[\mathrm{NO}_{2}\right]}\end{array}$} & HOMO to LUMO & 579 & 0.1373 & 1 \\
\hline & HOMO-1 to LUMO & 473 & 0.0031 & 2 \\
\hline \multirow{2}{*}{$\begin{array}{c}2 \mathrm{~B} \\
{\left[\mathrm{NO}_{2}\right]}\end{array}$} & HOMO to LUMO & 531 & 0.1491 & 1 \\
\hline & HOMO-2 to LUMO & 465 & 0.0037 & 2 \\
\hline \multirow{2}{*}{$\begin{array}{c}3 \mathrm{AB} \\
{[\mathrm{CHO}]}\end{array}$} & HOMO to LUMO & 567 & 0.1328 & 1 \\
\hline & HOMO-1 to LUMO & 510 & 0.0001 & 2 \\
\hline \multirow{2}{*}{$\begin{array}{c}\mathbf{3 A} \\
{[\mathrm{CHO}]}\end{array}$} & HOMO to LUMO & 589 & 0.1320 & 1 \\
\hline & HOMO-1 to LUMO & 480 & 0.0000 & 2 \\
\hline \multirow{2}{*}{$\begin{array}{c}\text { 3B } \\
{[\mathrm{CHO}]}\end{array}$} & HOMO to LUMO & 540 & 0.1429 & 1 \\
\hline & HOMO-1 to LUMO & 470 & 0.0005 & 2 \\
\hline \multirow{2}{*}{$\begin{array}{c}4 \mathrm{AB} \\
{[\mathrm{F}]}\end{array}$} & HOMO to LUMO & 541 & 0.1544 & 1 \\
\hline & HOMO-1 to LUMO & 461 & 0.0017 & 2 \\
\hline \multirow{2}{*}{$\begin{array}{l}4 \mathrm{~A} \\
{[\mathrm{~F}]}\end{array}$} & HOMO to LUMO & 544 & 0.1526 & 1 \\
\hline & HOMO-2 to LUMO & 451 & 0.0048 & 2 \\
\hline \multirow{2}{*}{$\begin{array}{l}\text { 4B } \\
{[F]}\end{array}$} & HOMO to LUMO & 557 & 0.1486 & 1 \\
\hline & HOMO-2 to LUMO & 455 & 0.0042 & 2 \\
\hline
\end{tabular}




\begin{tabular}{|c|c|c|c|c|}
\hline \multirow{2}{*}{$\begin{array}{l}5 \mathrm{AB} \\
{[\mathrm{Cl}]}\end{array}$} & HOMO to LUMO & 564 & 0.1646 & 1 \\
\hline & HOMO-2 to LUMO & 464 & 0.0058 & 2 \\
\hline \multirow{2}{*}{$\begin{array}{c}5 \mathrm{~A} \\
{[\mathrm{Cl}]}\end{array}$} & HOMO to LUMO & 556 & 0.1495 & 1 \\
\hline & HOMO-2 to LUMO & 456 & 0.0146 & 2 \\
\hline \multirow{2}{*}{$\begin{array}{c}5 B \\
{[\mathrm{Cl}]}\end{array}$} & HOMO to LUMO & 569 & 0.1572 & 1 \\
\hline & HOMO-2 to LUMO & 455 & 0.0057 & 2 \\
\hline \multirow{2}{*}{$\begin{array}{c}\mathbf{6 A B} \\
{\left[\mathrm{NH}_{2}\right]}\end{array}$} & HOMO to LUMO & 596 & 0.2466 & 1 \\
\hline & HOMO-1 to LUMO & 468 & 0.3357 & 2 \\
\hline \multirow{2}{*}{$\begin{array}{c}\mathbf{6 A} \\
{\left[\mathbf{N H}_{2}\right]}\end{array}$} & HOMO-1 to LUMO & 516 & 0.0233 & 1 \\
\hline & HOMO to LUMO & 495 & 0.5337 & 2 \\
\hline \multirow{2}{*}{$\begin{array}{c}\mathbf{6 B} \\
{\left[\mathrm{NH}_{2}\right]}\end{array}$} & HOMO to LUMO & 719 & 0.1759 & 1 \\
\hline & HOMO-1 to LUMO & 446 & 0.0079 & 2 \\
\hline \multirow{2}{*}{$\begin{array}{c}7 \mathrm{AB} \\
{\left[\mathrm{OCH}_{3}\right]}\end{array}$} & HOMO to LUMO & 573 & 0.2254 & 1 \\
\hline & HOMO-1 to LUMO & 460 & 0.3188 & 2 \\
\hline \multirow{2}{*}{$\begin{array}{c}7 \mathrm{~A} \\
{\left[\mathrm{OCH}_{3}\right]}\end{array}$} & HOMO to LUMO & 530 & 0.1859 & 1 \\
\hline & HOMO-1 to LUMO & 451 & 0.2812 & 2 \\
\hline \multirow{2}{*}{$\begin{array}{c}7 \mathrm{~B} \\
{\left[\mathrm{OCH}_{3}\right]}\end{array}$} & HOMO to LUMO & 612 & 0.1802 & 1 \\
\hline & HOMO-1 to LUMO & 447 & 0.0064 & 2 \\
\hline \multirow{2}{*}{$\begin{array}{c}\mathbf{8 A B} \\
{\left[\mathrm{NHCOCH}_{3}\right]}\end{array}$} & HOMO to LUMO & 633 & 0.1930 & 1 \\
\hline & HOMO-1 to LUMO & 470 & 0.1696 & 2 \\
\hline \multirow{2}{*}{$\begin{array}{c}\mathbf{8 A} \\
{\left[\mathrm{NHCPCH}_{3}\right]}\end{array}$} & HOMO to LUMO & 562 & 0.1270 & 1 \\
\hline & HOMO-1 to LUMO & 478 & 0.3296 & 2 \\
\hline \multirow{2}{*}{$\begin{array}{c}\mathbf{8 B} \\
{\left[\mathrm{NHCOCH}_{3}\right]}\end{array}$} & HOMO to LUMO & 629 & 0.1780 & 1 \\
\hline & HOMO-3 to LUMO & 449 & 0.0082 & 2 \\
\hline \multirow{2}{*}{$\begin{array}{c}\text { 9AB } \\
{\left[\mathrm{CH}_{2} \mathrm{CH}_{3}\right]}\end{array}$} & HOMO to LUMO & 568 & 0.1378 & 1 \\
\hline & HOMO-1 to LUMO & 448 & 0.0300 & 2 \\
\hline \multirow{2}{*}{$\begin{array}{c}9 \mathrm{~A} \\
{\left[\mathrm{CH}_{2} \mathrm{CH}_{3}\right]}\end{array}$} & HOMO to LUMO & 562 & 0.1442 & 1 \\
\hline & HOMO-2 to LUMO & 445 & 0.0245 & 2 \\
\hline 9B & HOMO to LUMO & 566 & 0.1387 & 1 \\
\hline
\end{tabular}




\begin{tabular}{lllll}
{$\left[\mathbf{C H}_{\mathbf{2}} \mathbf{C H}_{3}\right]$} & HOMO-2 to LUMO & 447 & 0.0211 & 2 \\
\hline
\end{tabular}


Table S4. Frontier orbitals involved and percent contribution of the orbitals for the $S_{0} \rightarrow S_{1}$ state transition at the optimized geometry of $S_{1}$ state, and at the optimized geometry of the $S_{0}$ state. Vertical excitation calculation was performed at TD-B3LYP/6-31+G(d,p) level of theory with the implicit solvation of acetonitrile.

\begin{tabular}{|l|l|l|l|l|}
\hline Compound & $\begin{array}{l}\mathrm{S}_{0} \rightarrow \mathrm{S}_{1} \text { Transition at the } \\
\text { geometry } \\
\text { Orbitals }\end{array}$ & $\begin{array}{l}\mathrm{S}_{1} \text { optimized } \\
\text { \% Contribution }\end{array}$ & $\begin{array}{l}\mathrm{S}_{0} \rightarrow \mathrm{S}_{1} \text { Transition at the } \\
\text { geometry } \\
\text { Orbitals }\end{array}$ \\
\hline $\mathrm{Et}-\mathrm{Fl}^{+}$ & HOMO to LUMO & $100 \%$ & $\begin{array}{l}\text { HOMO to LUMO } \\
\text { HOMO-1 to LUMO }\end{array}$ & $\begin{array}{l}\text { \% Contribution } \\
5 \%\end{array}$ \\
\hline $6 \mathrm{~A}$ & HOMO to LUMO & $18 \%$ & HOMO to LUMO & $5 \%$ \\
& HOMO-1 to LUMO & $82 \%$ & HOMO-1 to LUMO & $95 \%$ \\
\hline $6 \mathrm{~B}$ & HOMO to LUMO & $98 \%$ & HOMO to LUMO & $98 \%$ \\
\hline $6 \mathrm{AB}$ & HOMO to LUMO & $96 \%$ & HOMO to LUMO & $84 \%$ \\
& HOMO-1 to LUMO & $4 \%$ & HOMO-1 to LUMO & $16 \%$ \\
\hline
\end{tabular}




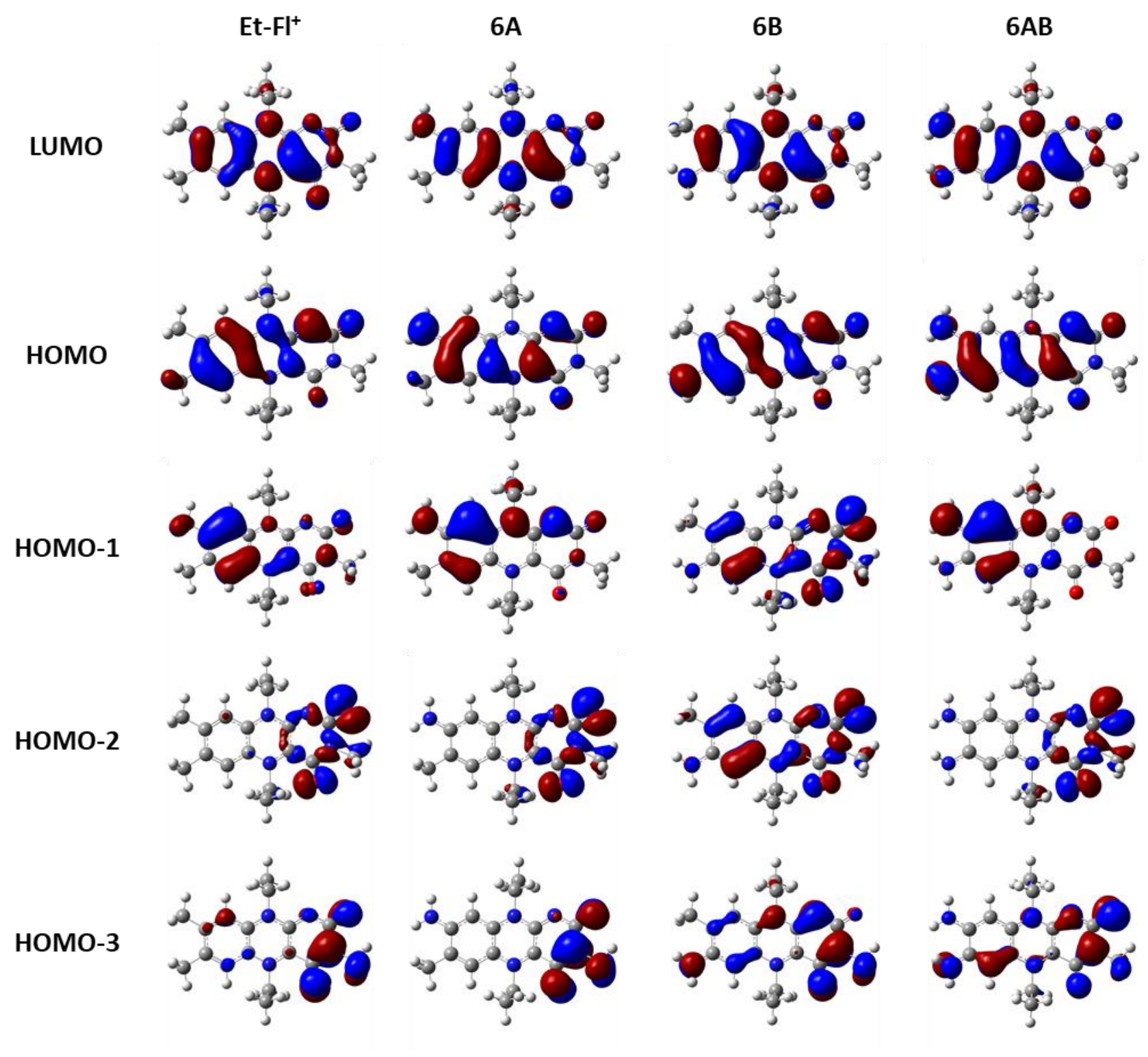

Figure S2. Frontier molecular orbitals for the parent $\mathrm{Et}_{-} \mathrm{Fl}^{+}, 6 \mathrm{~A}, 6 \mathrm{~B}$ and $6 \mathrm{AB}$ as calculated using B3LYP/6-31+G(d,p) level of theory with the implicit solvation of acetonitrile. All contours were prepared with an isoval of 0.003 a.u. 


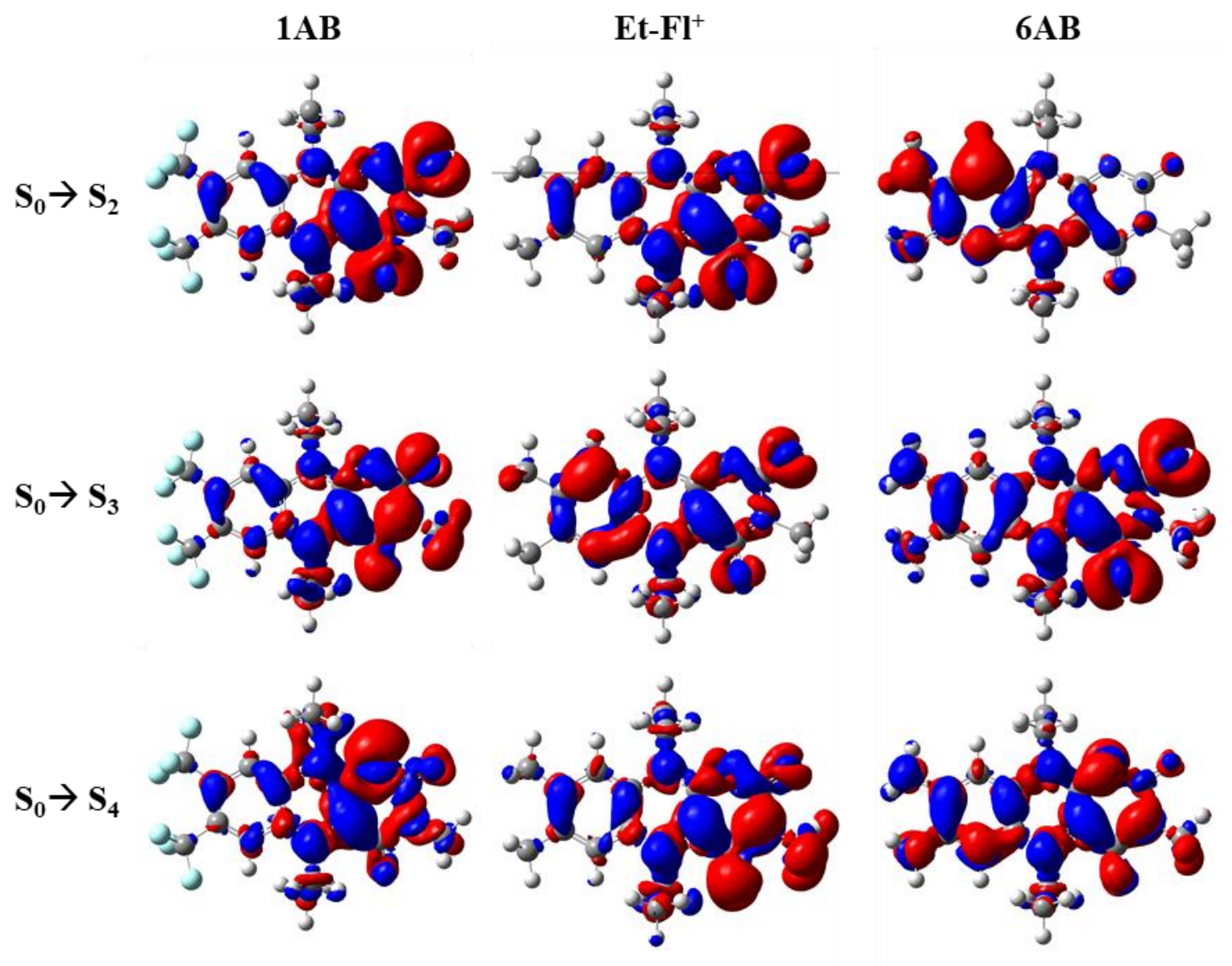

Figure S3. Difference density plots for the $S_{0} \rightarrow S_{2}, S_{0} \rightarrow S_{3}$, and $S_{0} \rightarrow S_{4}$ excited state transitions of $\mathrm{Et}_{-} \mathrm{Fl}^{+}$, and $1 \mathrm{AB}$ and $6 \mathrm{AB}$. The blue areas indicate electron accumulation and the red areas indicate election depletion upon excitation to the $S_{n}$ excited state. All contours prepared with an isoval of \pm 0.001 a.u. 

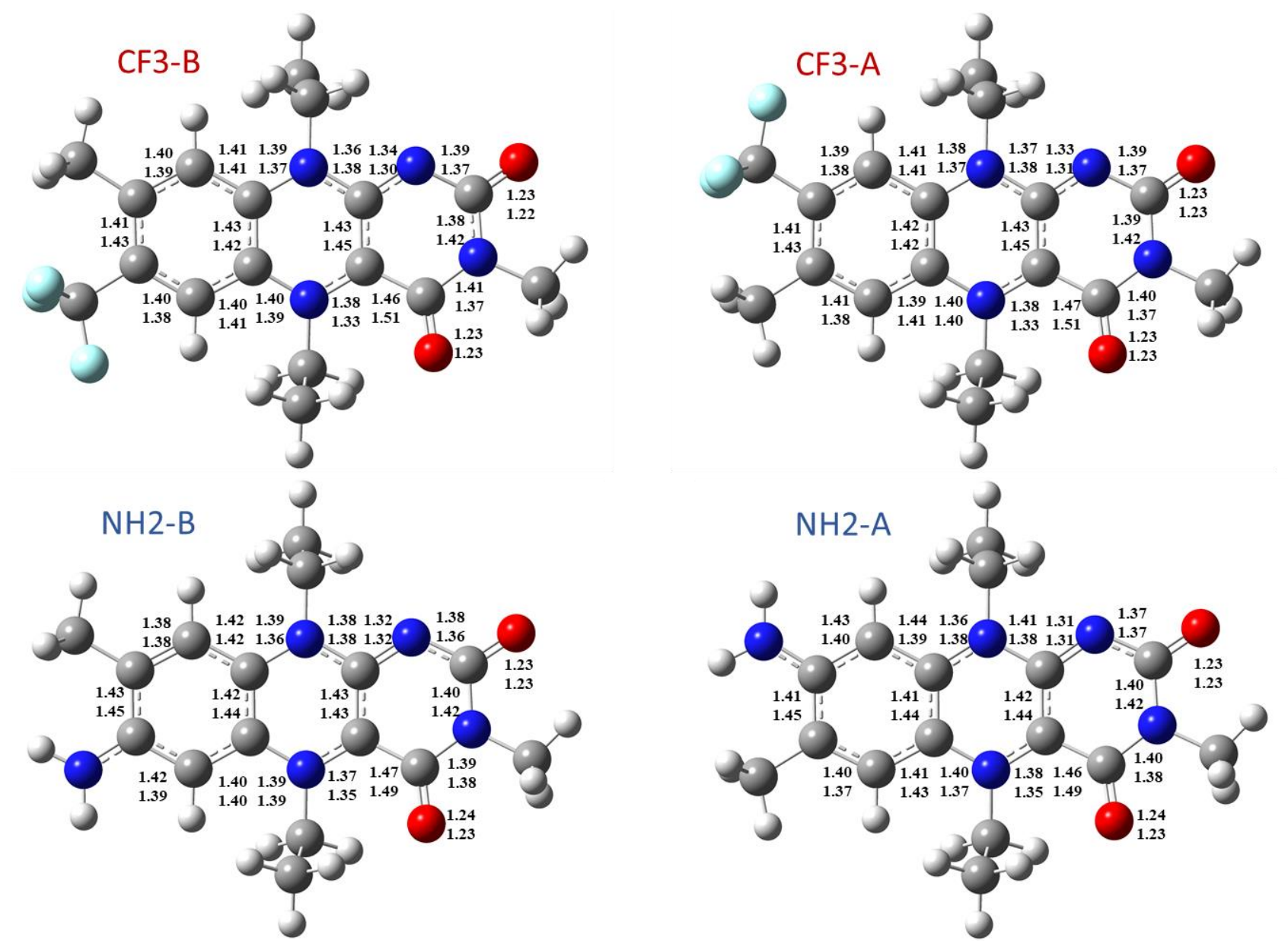

Figure S4. Optimized geometry at the B3LYP/6-31+G(d,p) level of theory for the $\mathrm{S}_{1}$ excited state (top value) and the $S_{0}$ ground state (bottom value) for 1A (top right), 1B (top left), 6A (bottom right), and 6B (bottom left). Bond lengths are presented in Angstrom. 


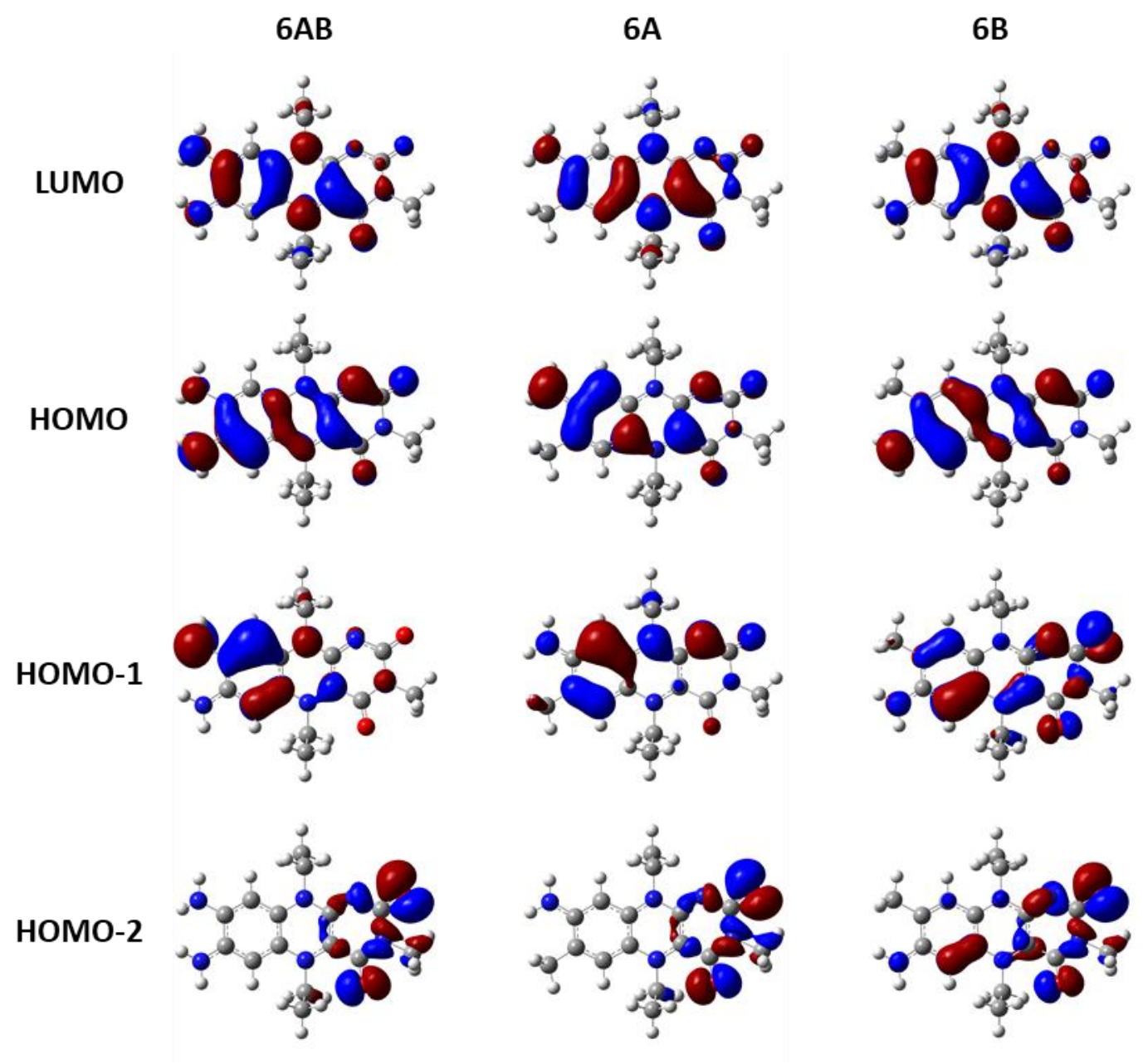

Figure S5. Frontier orbitals at the optimized $S_{1}$ state geometries for $6 A B, 6 A$ and $6 B$ at TDB3LYP/6-31+G(d,p) level of theory with implicit solvation of acetonitrile. All contours were prepared with an isoval of 0.003 . 
Table S5. Cartesian coordinates for all ground state $\mathrm{Et}^{-\mathrm{Fl}^{+}}$derivatives optimized at B3LYP/6$31+\mathrm{G}(\mathrm{d}, \mathrm{p})$ with implicit solvation of acetonitrile.

Table S5a. Cartesian coordinates for Et-Fl .

\begin{tabular}{|c|c|c|c|}
\hline \multirow[t]{2}{*}{ Atom } & \multicolumn{3}{|c|}{ Coordinates (Angstroms) } \\
\hline & $X$ & $\mathrm{Y}$ & $\mathrm{Z}$ \\
\hline $\mathrm{C}$ & -3.65960900 & -0.79628100 & -0.13065500 \\
\hline $\mathrm{C}$ & -2.42119700 & -1.39334100 & -0.24124000 \\
\hline $\mathrm{C}$ & -1.21994600 & -0.65543200 & -0.11204400 \\
\hline $\mathrm{C}$ & -1.29535000 & 0.74841500 & 0.14777900 \\
\hline $\mathrm{C}$ & -2.56622000 & 1.34817900 & 0.24567700 \\
\hline $\mathrm{C}$ & -3.73290900 & 0.61496400 & 0.11165800 \\
\hline $\mathrm{C}$ & 1.09722600 & 0.88954700 & 0.11773800 \\
\hline $\mathrm{C}$ & 1.14606500 & -0.54547300 & -0.05557300 \\
\hline $\mathrm{C}$ & 2.51204700 & -1.16519800 & -0.01942200 \\
\hline $\mathrm{C}$ & 3.41359400 & 1.13795500 & 0.00912300 \\
\hline $\mathrm{H}$ & -2.39046200 & -2.45798400 & -0.41432400 \\
\hline $\mathrm{H}$ & -2.64756800 & 2.41191600 & 0.41495100 \\
\hline $\mathrm{N}$ & 3.55816600 & -0.27515200 & -0.04996500 \\
\hline $\mathrm{N}$ & 2.15610000 & 1.65974300 & 0.12766400 \\
\hline $\mathrm{N}$ & -0.13767700 & 1.47830300 & 0.27287900 \\
\hline O & 2.70764600 & -2.37596300 & 0.05116500 \\
\hline 0 & 4.41246900 & 1.85066000 & -0.03274600 \\
\hline $\mathrm{C}$ & -4.91290200 & -1.61840900 & -0.26410100 \\
\hline $\mathrm{H}$ & -5.52477700 & -1.55347900 & 0.64244200 \\
\hline $\mathrm{H}$ & -5.53229700 & -1.25917200 & -1.09354400 \\
\hline $\mathrm{H}$ & -4.67810900 & -2.66915500 & -0.44391800 \\
\hline $\mathrm{C}$ & -5.06532400 & 1.29984800 & 0.21626700 \\
\hline $\mathrm{H}$ & -5.64528000 & 1.16510700 & -0.70377600 \\
\hline $\mathrm{H}$ & -5.66007900 & 0.87068800 & 1.03052700 \\
\hline $\mathrm{H}$ & -4.95064900 & 2.36944400 & 0.39892200 \\
\hline $\mathrm{C}$ & -0.17292800 & 2.94268400 & 0.54466400 \\
\hline $\mathrm{H}$ & -1.00131900 & 3.12561200 & 1.22679500 \\
\hline $\mathrm{H}$ & 0.75312900 & 3.16921100 & 1.06849700 \\
\hline $\mathrm{C}$ & -0.28008500 & 3.76892500 & -0.73760500 \\
\hline $\mathrm{H}$ & 0.58239200 & 3.59160700 & -1.38417200 \\
\hline $\mathrm{H}$ & -1.19283500 & 3.54287600 & -1.29544600 \\
\hline $\mathrm{H}$ & -0.29701500 & 4.82856900 & -0.46741400 \\
\hline $\mathrm{C}$ & 0.06415000 & -2.73220800 & -0.55166000 \\
\hline $\mathrm{H}$ & -0.73027800 & -2.88441900 & -1.27773000 \\
\hline $\mathrm{H}$ & 1.01103800 & -2.91584700 & -1.04338500 \\
\hline $\mathrm{C}$ & -0.08652000 & -3.61718400 & 0.68322600 \\
\hline $\mathrm{H}$ & 0.74830300 & -3.46816900 & 1.36979600 \\
\hline $\mathrm{H}$ & -1.02477000 & -3.43899400 & 1.21348100 \\
\hline $\mathrm{H}$ & -0.07450900 & -4.65823000 & 0.34790000 \\
\hline
\end{tabular}




$\begin{array}{lllr}\mathrm{N} & 0.02640300 & -1.25819100 & -0.21260100 \\ \mathrm{C} & 4.90875800 & -0.85988200 & -0.05359900 \\ \mathrm{H} & 4.99248600 & -1.57006100 & -0.87750500 \\ \mathrm{H} & 5.61949100 & -0.04810600 & -0.17439100 \\ \mathrm{H} & 5.08814500 & -1.38455500 & 0.88772100\end{array}$

Table S5b. Cartesian coordinates for 1AB.

\begin{tabular}{|c|c|c|c|}
\hline \multirow[t]{2}{*}{ Atom } & \multicolumn{3}{|c|}{ Coordinates (Angstroms) } \\
\hline & $X$ & $\mathrm{Y}$ & $\mathrm{Z}$ \\
\hline C & -2.46101200 & -0.71253800 & 0.12966200 \\
\hline C & -1.23026600 & -1.32943700 & 0.22995800 \\
\hline C & -0.02810200 & -0.60886300 & 0.09305900 \\
\hline C & -0.07987200 & 0.78856000 & -0.14588100 \\
\hline C & -1.34515200 & 1.40573800 & -0.25108300 \\
\hline C & -2.51502300 & 0.68599400 & -0.12374700 \\
\hline $\mathrm{C}$ & 2.30329300 & 0.90700400 & -0.07672100 \\
\hline $\mathrm{C}$ & 2.33114900 & -0.54643800 & 0.01840000 \\
\hline $\mathrm{C}$ & 3.69734400 & -1.18165800 & -0.08175600 \\
\hline $\mathrm{C}$ & 4.62191700 & 1.09707300 & 0.12060900 \\
\hline $\mathrm{H}$ & -1.21401800 & -2.39136200 & 0.40958400 \\
\hline $\mathrm{H}$ & -1.41005200 & 2.46809500 & -0.42348400 \\
\hline $\mathrm{N}$ & 4.75131300 & -0.31598000 & 0.05295100 \\
\hline $\mathrm{N}$ & 3.36820900 & 1.64905500 & -0.00485200 \\
\hline $\mathrm{N}$ & 1.21803600 & -1.24905500 & 0.15953900 \\
\hline $\mathrm{N}$ & 1.07990200 & 1.51579300 & -0.25059500 \\
\hline 0 & 3.85880900 & -2.37850700 & -0.28521000 \\
\hline 0 & 5.61616600 & 1.79338200 & 0.26544900 \\
\hline $\mathrm{C}$ & -3.81556500 & 1.47682300 & -0.21985500 \\
\hline $\mathrm{C}$ & -3.68822700 & -1.60106200 & 0.26294500 \\
\hline $\mathrm{F}$ & -3.61708100 & 2.68674800 & -0.78704800 \\
\hline $\mathrm{F}$ & -4.34610600 & 1.69984700 & 1.00472900 \\
\hline $\mathrm{F}$ & -4.64120800 & -1.04673600 & 1.04254900 \\
\hline $\mathrm{F}$ & -3.37362500 & -2.79475800 & 0.81534100 \\
\hline $\mathrm{F}$ & -4.24912400 & -1.86573700 & -0.94106100 \\
\hline $\mathrm{F}$ & -4.74591400 & 0.84236200 & -0.96251700 \\
\hline $\mathrm{C}$ & 1.06939300 & 2.98710200 & -0.49524600 \\
\hline $\mathrm{H}$ & 2.01100400 & 3.20696400 & -0.99461700 \\
\hline $\mathrm{H}$ & 0.26268400 & 3.19303400 & -1.19703400 \\
\hline $\mathrm{C}$ & 0.94448300 & 3.79470300 & 0.79706400 \\
\hline $\mathrm{H}$ & 0.01283300 & 3.58149700 & 1.32813500 \\
\hline $\mathrm{H}$ & 1.78568100 & 3.59151200 & 1.46330300 \\
\hline $\mathrm{H}$ & 0.95589600 & 4.85748500 & 0.54001500 \\
\hline $\mathrm{C}$ & 6.09715500 & -0.91479100 & 0.02154400 \\
\hline $\mathrm{H}$ & 6.30442900 & -1.30608000 & -0.97709400 \\
\hline $\mathrm{H}$ & 6.81084700 & -0.13792900 & 0.27815200 \\
\hline
\end{tabular}




$\begin{array}{llrr}\mathrm{H} & 6.14074400 & -1.73147700 & 0.74289800 \\ \mathrm{C} & 1.23204800 & -2.74162100 & 0.42387100 \\ \mathrm{H} & 2.19392400 & -2.97020400 & 0.86482200 \\ \mathrm{H} & 0.46803200 & -2.90813100 & 1.17966400 \\ \mathrm{C} & 1.00626700 & -3.55954400 & -0.84587500 \\ \mathrm{H} & 1.02130400 & -4.61497000 & -0.56024600 \\ \mathrm{H} & 0.04530100 & -3.34789200 & -1.31975300 \\ \mathrm{H} & 1.80791100 & -3.38604200 & -1.56518900\end{array}$

Table S5c. Cartesian coordinates for $2 \mathrm{AB}$.

\begin{tabular}{|c|c|c|c|}
\hline \multirow[t]{2}{*}{ Atom } & \multicolumn{3}{|c|}{ Coordinates (Angstroms) } \\
\hline & $X$ & $\mathrm{Y}$ & $\mathrm{Z}$ \\
\hline $\mathrm{C}$ & -1.64546900 & 1.36655900 & 0.23351100 \\
\hline $\mathrm{C}$ & -0.45780600 & 0.62581900 & 0.10530100 \\
\hline $\mathrm{C}$ & -0.52344900 & -0.77470100 & -0.14562500 \\
\hline $\mathrm{C}$ & -1.79339500 & -1.39091500 & -0.23955300 \\
\hline $\mathrm{C}$ & 1.85825800 & -0.91000600 & -0.09034600 \\
\hline $\mathrm{C}$ & 1.90050300 & 0.54327600 & 0.02780700 \\
\hline $\mathrm{C}$ & 3.27497900 & 1.16703400 & -0.05734700 \\
\hline $\mathrm{C}$ & 4.17709600 & -1.12305000 & 0.09388500 \\
\hline $\mathrm{H}$ & -1.64785500 & 2.43379900 & 0.39015200 \\
\hline $\mathrm{H}$ & -1.88674100 & -2.45630900 & -0.38780800 \\
\hline $\mathrm{N}$ & 4.32036300 & 0.28780200 & 0.05473200 \\
\hline $\mathrm{N}$ & 2.91386100 & -1.66099300 & -0.03653200 \\
\hline $\mathrm{N}$ & 0.79633200 & 1.25415900 & 0.17753100 \\
\hline $\mathrm{N}$ & 0.62659600 & -1.50750200 & -0.26677300 \\
\hline O & 3.44641900 & 2.36621400 & -0.23082200 \\
\hline O & 5.16074400 & -1.83532800 & 0.21964100 \\
\hline C & -2.86935200 & 0.74576400 & 0.12315400 \\
\hline C & -2.93490000 & -0.64220600 & -0.10041900 \\
\hline $\mathrm{N}$ & -4.07169700 & 1.58675100 & 0.16440600 \\
\hline O & -3.98229100 & 2.68264800 & 0.71219200 \\
\hline O & -5.08295300 & 1.14354800 & -0.37807500 \\
\hline $\mathrm{C}$ & 0.60325300 & -2.97558500 & -0.53711700 \\
\hline $\mathrm{H}$ & -0.21501800 & -3.16246700 & -1.23105500 \\
\hline $\mathrm{H}$ & 1.53536400 & -3.19110100 & -1.05606700 \\
\hline C & 0.49440600 & -3.80483000 & 0.74259700 \\
\hline $\mathrm{H}$ & 1.34968400 & -3.62169000 & 1.39638000 \\
\hline $\mathrm{H}$ & -0.42486600 & -3.59316200 & 1.29529400 \\
\hline $\mathrm{H}$ & 0.49078500 & -4.86253300 & 0.46546000 \\
\hline C & 0.82082700 & 2.74466100 & 0.46146000 \\
\hline $\mathrm{H}$ & 0.04819900 & 2.90755600 & 1.20919800 \\
\hline $\mathrm{H}$ & 1.77871900 & 2.95645100 & 0.91953200 \\
\hline C & 0.62170900 & 3.58197900 & -0.79986000 \\
\hline $\mathrm{H}$ & 1.43046100 & 3.40959400 & -1.51122700 \\
\hline
\end{tabular}




$\begin{array}{rrrr}\mathrm{H} & -0.33522000 & 3.39015200 & -1.29021400 \\ \mathrm{H} & 0.64567000 & 4.63259900 & -0.49766300 \\ \mathrm{C} & 5.67267800 & 0.87388200 & 0.03463000 \\ \mathrm{H} & 6.37913600 & 0.08392300 & 0.27025600 \\ \mathrm{H} & 5.88059700 & 1.28698400 & -0.95492400 \\ \mathrm{H} & 5.72574300 & 1.67193800 & 0.77581000 \\ \mathrm{~N} & -4.21604700 & -1.38513000 & -0.10113200 \\ \mathrm{O} & -4.85960300 & -1.36480700 & 0.94101300 \\ \mathrm{O} & -4.49938500 & -2.00195600 & -1.12034900\end{array}$

Table S5d. Cartesian coordinates for 3AB.

\begin{tabular}{|c|c|c|c|}
\hline \multirow[t]{2}{*}{ Atom } & \multicolumn{3}{|c|}{ Coordinates (Angstroms) } \\
\hline & $X$ & $\mathrm{Y}$ & $\mathrm{Z}$ \\
\hline $\mathrm{C}$ & 3.32635600 & -0.77614200 & 0.14217000 \\
\hline C & 2.08155100 & -1.37866200 & 0.24612100 \\
\hline $\mathrm{C}$ & 0.89587000 & -0.64036900 & 0.10949400 \\
\hline $\mathrm{C}$ & 0.96858900 & 0.76351800 & -0.13677200 \\
\hline C & 2.23740000 & 1.36461200 & -0.23336200 \\
\hline $\mathrm{C}$ & 3.40051100 & 0.62450900 & -0.09941700 \\
\hline $\mathrm{C}$ & -1.41680000 & 0.90928200 & -0.08123400 \\
\hline C & -1.46527100 & -0.54303100 & 0.02804900 \\
\hline C & -2.83948900 & -1.16094700 & -0.07004300 \\
\hline $\mathrm{C}$ & -3.73471700 & 1.13195300 & 0.09905800 \\
\hline $\mathrm{H}$ & 2.07480600 & -2.44405000 & 0.42156200 \\
\hline $\mathrm{H}$ & 2.34057800 & 2.42735300 & -0.39892400 \\
\hline $\mathrm{N}$ & -3.88296800 & -0.27981000 & 0.04476600 \\
\hline $\mathrm{N}$ & -2.47360700 & 1.66585900 & -0.02295000 \\
\hline $\mathrm{N}$ & -0.36241400 & -1.25946700 & 0.17741600 \\
\hline $\mathrm{N}$ & -0.18622500 & 1.50053400 & -0.25164300 \\
\hline O & -3.01683200 & -2.35850300 & -0.25658200 \\
\hline O & -4.72111800 & 1.84275100 & 0.23013500 \\
\hline C & 4.51965300 & -1.66457500 & 0.28342200 \\
\hline $\mathrm{H}$ & 5.51301200 & -1.20778500 & 0.16777100 \\
\hline C & 4.69645400 & 1.37604200 & -0.20341100 \\
\hline $\mathrm{H}$ & 5.62686400 & 0.81331400 & -0.04351300 \\
\hline O & 4.42024800 & -2.85878700 & 0.51330400 \\
\hline 0 & 4.73556200 & 2.56839200 & -0.44917600 \\
\hline C & -0.15458200 & 2.96895300 & -0.50841400 \\
\hline $\mathrm{H}$ & 0.66376200 & 3.15716800 & -1.20152300 \\
\hline $\mathrm{H}$ & -1.08766600 & 3.19794300 & -1.01965300 \\
\hline C & -0.03158100 & 3.78560200 & 0.77824500 \\
\hline $\mathrm{H}$ & -0.88286400 & 3.60081200 & 1.43711900 \\
\hline $\mathrm{H}$ & 0.89109000 & 3.56154200 & 1.32034300 \\
\hline $\mathrm{H}$ & -0.02360100 & 4.84647300 & 0.51305500 \\
\hline C & -5.23620500 & -0.86133600 & 0.01127200 \\
\hline
\end{tabular}




$\begin{array}{lrrr}\mathrm{H} & -5.29878300 & -1.66270300 & 0.74834300 \\ \mathrm{H} & -5.94196200 & -0.07000400 & 0.24448100 \\ \mathrm{H} & -5.43821300 & -1.27014300 & -0.98138900 \\ \mathrm{C} & -0.39672400 & -2.74885200 & 0.45500700 \\ \mathrm{H} & -1.35944600 & -2.96016700 & 0.90286000 \\ \mathrm{H} & 0.37051700 & -2.91923100 & 1.20626700 \\ \mathrm{C} & -0.18808400 & -3.58155800 & -0.80789200 \\ \mathrm{H} & -0.21817400 & -4.63413900 & -0.51284800 \\ \mathrm{H} & -0.98883600 & -3.40265500 & -1.52688400 \\ \mathrm{H} & 0.77496600 & -3.38838100 & -1.28541700\end{array}$

Table S5e. Cartesian coordinates for $4 \mathrm{AB}$.

\begin{tabular}{|c|c|c|c|}
\hline \multirow[t]{2}{*}{ Atom } & \multicolumn{3}{|c|}{ Coordinates (Angstroms) } \\
\hline & $X$ & $\mathrm{Y}$ & $\mathrm{Z}$ \\
\hline $\mathrm{C}$ & 2.43581400 & -1.41219100 & 0.26787100 \\
\hline $\mathrm{C}$ & 1.24792400 & -0.65803600 & 0.12043400 \\
\hline $\mathrm{C}$ & 1.32751700 & 0.74332600 & -0.15497900 \\
\hline $\mathrm{C}$ & 2.59539000 & 1.35248900 & -0.26625300 \\
\hline C & -1.06183100 & 0.89452200 & -0.11882400 \\
\hline $\mathrm{C}$ & -1.11448900 & -0.54482100 & 0.05286900 \\
\hline C & -2.48624000 & -1.16199100 & 0.00376400 \\
\hline $\mathrm{C}$ & -3.37822200 & 1.14244300 & 0.00845400 \\
\hline $\mathrm{H}$ & 2.43634000 & -2.47380900 & 0.45980100 \\
\hline $\mathrm{H}$ & 2.71436200 & 2.40963000 & -0.45081200 \\
\hline $\mathrm{N}$ & -3.52806500 & -0.27019500 & 0.05058000 \\
\hline $\mathrm{N}$ & -2.11628800 & 1.66289600 & -0.11616600 \\
\hline $\mathrm{N}$ & -0.00463000 & -1.26009000 & 0.21871600 \\
\hline $\mathrm{N}$ & 0.17463100 & 1.47817300 & -0.28483200 \\
\hline O & -2.67677200 & -2.37003300 & -0.09075200 \\
\hline O & -4.36958000 & 1.85985200 & 0.06709100 \\
\hline $\mathrm{C}$ & 3.64921500 & -0.79113600 & 0.14603700 \\
\hline C & 3.72670200 & 0.59020200 & -0.11857200 \\
\hline $\mathrm{F}$ & 4.78702200 & -1.48482500 & 0.27449800 \\
\hline $\mathrm{F}$ & 4.93299800 & 1.15186300 & -0.21636000 \\
\hline C & 0.21415100 & 2.94280300 & -0.56066400 \\
\hline $\mathrm{H}$ & 1.03801300 & 3.12077800 & -1.24989500 \\
\hline $\mathrm{H}$ & -0.71371400 & 3.16937200 & -1.08155600 \\
\hline C & 0.32907200 & 3.77178600 & 0.71912000 \\
\hline $\mathrm{H}$ & -0.53213800 & 3.60014800 & 1.36864800 \\
\hline $\mathrm{H}$ & 1.24243400 & 3.54518100 & 1.27578900 \\
\hline $\mathrm{H}$ & 0.34932500 & 4.83000300 & 0.44423000 \\
\hline C & -4.88183000 & -0.84968100 & 0.04726900 \\
\hline $\mathrm{H}$ & -4.95745700 & -1.58937400 & 0.84521300 \\
\hline $\mathrm{H}$ & -5.58797300 & -0.04053000 & 0.20631900 \\
\hline $\mathrm{H}$ & -5.07321100 & -1.33735100 & -0.91141400 \\
\hline
\end{tabular}




$\begin{array}{lrrr}\mathrm{C} & -0.04545000 & -2.73572900 & 0.56058500 \\ \mathrm{H} & 0.73691900 & -2.88286000 & 1.30115700 \\ \mathrm{H} & -0.99888100 & -2.91766100 & 1.04015400 \\ \mathrm{C} & 0.12311400 & -3.62394200 & -0.66956700 \\ \mathrm{H} & 0.10925900 & -4.66276500 & -0.32785900 \\ \mathrm{H} & -0.70398200 & -3.48007900 & -1.36620200 \\ \mathrm{H} & 1.06679100 & -3.44832900 & -1.19089900\end{array}$

Table S5f. Cartesian coordinates for $5 \mathrm{AB}$.

\begin{tabular}{|c|c|c|c|}
\hline \multirow[t]{2}{*}{ Atom } & \multicolumn{3}{|c|}{ Coordinates (Angstroms) } \\
\hline & $X$ & $\mathrm{Y}$ & $\mathrm{Z}$ \\
\hline $\mathrm{C}$ & -1.96520900 & -1.36318800 & -0.24265000 \\
\hline C & -0.76726500 & -0.63001800 & -0.10958500 \\
\hline $\mathrm{C}$ & -0.82948900 & 0.77246900 & 0.14578100 \\
\hline $\mathrm{C}$ & -2.09118700 & 1.38891900 & 0.24771600 \\
\hline $\mathrm{C}$ & 1.55980800 & 0.89682000 & 0.10215300 \\
\hline $\mathrm{C}$ & 1.59549200 & -0.54724600 & -0.04382700 \\
\hline $\mathrm{C}$ & 2.96030000 & -1.17934800 & 0.01928900 \\
\hline $\mathrm{C}$ & 3.87916400 & 1.11310600 & -0.03871000 \\
\hline $\mathrm{H}$ & -1.95688200 & -2.42665200 & -0.41978000 \\
\hline $\mathrm{H}$ & -2.17649000 & 2.45116900 & 0.41738600 \\
\hline $\mathrm{N}$ & 4.01267900 & -0.30160500 & -0.04789600 \\
\hline $\mathrm{N}$ & 2.62373500 & 1.65123600 & 0.08084100 \\
\hline $\mathrm{N}$ & 0.47816100 & -1.25202000 & -0.19832600 \\
\hline $\mathrm{N}$ & 0.33176700 & 1.49752900 & 0.26539300 \\
\hline 0 & 3.13542700 & -2.38712600 & 0.14150400 \\
\hline 0 & 4.87797700 & 1.81767100 & -0.11908300 \\
\hline $\mathrm{Cl}$ & -4.63319400 & -1.69235000 & -0.29185900 \\
\hline $\mathrm{C}$ & -3.19107100 & -0.74354100 & -0.13034200 \\
\hline $\mathrm{Cl}$ & -4.77310000 & 1.46634000 & 0.24529400 \\
\hline $\mathrm{C}$ & -3.25270300 & 0.65292800 & 0.11520400 \\
\hline C & 0.31032200 & 2.96435900 & 0.52948600 \\
\hline $\mathrm{H}$ & -0.50806300 & 3.15697700 & 1.22146500 \\
\hline $\mathrm{H}$ & 1.24331700 & 3.18435100 & 1.04417000 \\
\hline $\mathrm{C}$ & 0.19838300 & 3.78645000 & -0.75495200 \\
\hline $\mathrm{H}$ & 1.05279300 & 3.59889800 & -1.40905300 \\
\hline $\mathrm{H}$ & -0.72169700 & 3.56891400 & -1.30410200 \\
\hline $\mathrm{H}$ & 0.19439800 & 4.84663200 & -0.48691900 \\
\hline $\mathrm{C}$ & 5.35955900 & -0.89687100 & -0.03356900 \\
\hline $\mathrm{H}$ & 5.42463200 & -1.65573700 & -0.81425100 \\
\hline $\mathrm{H}$ & 6.07487200 & -0.10007400 & -0.21280200 \\
\hline $\mathrm{H}$ & 5.54717100 & -1.36432300 & 0.93585600 \\
\hline $\mathrm{C}$ & 0.50105000 & -2.73327800 & -0.51538200 \\
\hline $\mathrm{H}$ & -0.27983600 & -2.88275700 & -1.25710200 \\
\hline $\mathrm{H}$ & 1.45420200 & -2.93572900 & -0.98707600 \\
\hline
\end{tabular}




$\begin{array}{lrrr}\mathrm{C} & 0.31549600 & -3.59944100 & 0.72812700 \\ \mathrm{H} & 0.32787400 & -4.64399600 & 0.40436400 \\ \mathrm{H} & 1.13589900 & -3.44775800 & 1.43107600 \\ \mathrm{H} & -0.63271700 & -3.41079800 & 1.23646600\end{array}$

Table S5g. Cartesian coordinates for 6AB.

\begin{tabular}{|c|c|c|c|}
\hline \multirow[t]{2}{*}{ Atom } & \multicolumn{3}{|c|}{ Coordinates (Angstroms) } \\
\hline & $X$ & $\mathrm{Y}$ & $\mathrm{Z}$ \\
\hline $\mathrm{C}$ & -2.43973000 & -1.41228700 & -0.17650300 \\
\hline C & -1.23943300 & -0.66877800 & -0.10493500 \\
\hline C & -1.31599600 & 0.75640700 & 0.13391300 \\
\hline $\mathrm{C}$ & -2.56935600 & 1.36645400 & 0.20354800 \\
\hline C & 1.08584300 & 0.87384300 & 0.11814200 \\
\hline $\mathrm{C}$ & 1.13611400 & -0.54097000 & -0.07856600 \\
\hline $\mathrm{C}$ & 2.48452100 & -1.15841600 & -0.07739900 \\
\hline C & 3.39902800 & 1.13930000 & 0.08265400 \\
\hline $\mathrm{H}$ & -2.41542400 & -2.48658000 & -0.27280700 \\
\hline $\mathrm{H}$ & -2.65406600 & 2.43821800 & 0.30239100 \\
\hline $\mathrm{N}$ & 3.53910500 & -0.26776000 & -0.03117700 \\
\hline $\mathrm{N}$ & 2.14358700 & 1.65661200 & 0.17739100 \\
\hline $\mathrm{N}$ & -0.01021000 & -1.25891100 & -0.22698500 \\
\hline $\mathrm{N}$ & -0.15488100 & 1.47405700 & 0.25136600 \\
\hline 0 & 2.69938100 & -2.37334300 & -0.09808500 \\
\hline O & 4.40656100 & 1.85127400 & 0.10350000 \\
\hline C & -3.67850200 & -0.81592200 & -0.05214900 \\
\hline C & -3.75036600 & 0.63230400 & 0.07760500 \\
\hline $\mathrm{N}$ & -4.84462300 & -1.53273200 & -0.11039600 \\
\hline $\mathrm{H}$ & -4.76130600 & -2.54100600 & -0.13549400 \\
\hline $\mathrm{H}$ & -5.62872500 & -1.22350300 & 0.45000000 \\
\hline $\mathrm{N}$ & -4.95504300 & 1.23501900 & 0.09739000 \\
\hline $\mathrm{H}$ & -5.78415300 & 0.76248100 & -0.23330500 \\
\hline $\mathrm{H}$ & -5.01635300 & 2.24278600 & 0.14895300 \\
\hline C & 4.88559800 & -0.85704600 & -0.04462500 \\
\hline $\mathrm{H}$ & 5.60195800 & -0.04105400 & -0.04181500 \\
\hline $\mathrm{H}$ & 5.02408500 & -1.48744300 & 0.83673200 \\
\hline $\mathrm{H}$ & 5.00882100 & -1.47089100 & -0.93882500 \\
\hline $\mathrm{C}$ & -0.19036800 & 2.93722300 & 0.51990000 \\
\hline $\mathrm{H}$ & -1.02807800 & 3.12327500 & 1.19078900 \\
\hline $\mathrm{H}$ & 0.72812600 & 3.16487700 & 1.05549100 \\
\hline $\mathrm{C}$ & -0.27814400 & 3.76404000 & -0.76373800 \\
\hline $\mathrm{H}$ & 0.59744500 & 3.58896600 & -1.39330800 \\
\hline $\mathrm{H}$ & -1.17820200 & 3.53500500 & -1.34087500 \\
\hline $\mathrm{H}$ & -0.30410700 & 4.82434600 & -0.49612300 \\
\hline $\mathrm{C}$ & 0.02711300 & -2.72225600 & -0.58760500 \\
\hline $\mathrm{H}$ & -0.79642900 & -2.87848400 & -1.28093000 \\
\hline
\end{tabular}




$\begin{array}{lrrr}\text { H } & 0.95473900 & -2.88968400 & -1.12095800 \\ \mathrm{C} & -0.05742500 & -3.62963100 & 0.63810400 \\ \mathrm{H} & 0.81266000 & -3.48749100 & 1.28103600 \\ \mathrm{H} & -0.96534500 & -3.45989700 & 1.22189900 \\ \mathrm{H} & -0.06210800 & -4.66695900 & 0.29064100\end{array}$

Table S5h. Cartesian coordinates for 7AB.

\begin{tabular}{|c|c|c|c|}
\hline \multirow[t]{2}{*}{ Atom } & \multicolumn{3}{|c|}{ Coordinates (Angstroms) } \\
\hline & $X$ & $\mathrm{Y}$ & $\mathrm{Z}$ \\
\hline $\mathrm{C}$ & -3.22423700 & -0.53063500 & -0.05069400 \\
\hline C & -2.02407400 & -1.20081500 & -0.16452500 \\
\hline C & -0.78251800 & -0.52718300 & -0.07138200 \\
\hline C & -0.77777800 & 0.89103600 & 0.16092500 \\
\hline C & -2.00093100 & 1.56490000 & 0.24898700 \\
\hline $\mathrm{C}$ & -3.21850000 & 0.90387300 & 0.14192400 \\
\hline C & 1.62102100 & 0.88839000 & 0.11311300 \\
\hline C & 1.58962900 & -0.53969700 & -0.05204300 \\
\hline $\mathrm{C}$ & 2.91077900 & -1.23177900 & -0.03842100 \\
\hline C & 3.94505800 & 1.01720400 & 0.00909600 \\
\hline $\mathrm{H}$ & -2.05195800 & -2.26860100 & -0.29528300 \\
\hline $\mathrm{H}$ & -2.04738900 & 2.63506400 & 0.38169700 \\
\hline $\mathrm{N}$ & 4.00830200 & -0.39966900 & -0.05960200 \\
\hline $\mathrm{N}$ & 2.71992200 & 1.60612600 & 0.12515100 \\
\hline $\mathrm{N}$ & 0.41676200 & -1.19322600 & -0.18287400 \\
\hline $\mathrm{N}$ & 0.41944400 & 1.55199600 & 0.26334300 \\
\hline O & 3.04976700 & -2.45421200 & 0.00580700 \\
\hline O & 4.98520500 & 1.67377300 & -0.02475500 \\
\hline 0 & -4.29016300 & 1.68517100 & 0.25320300 \\
\hline 0 & -4.42643600 & -1.12182300 & -0.09177300 \\
\hline C & -4.51562300 & -2.54542700 & -0.28509000 \\
\hline $\mathrm{H}$ & -4.03283600 & -3.07423000 & 0.54176100 \\
\hline $\mathrm{H}$ & -5.58147400 & -2.76510000 & -0.29468000 \\
\hline $\mathrm{H}$ & -4.06620600 & -2.83088000 & -1.24072900 \\
\hline C & -5.66339400 & 1.29416600 & -0.00832200 \\
\hline $\mathrm{H}$ & -6.01360500 & 0.59696000 & 0.75152400 \\
\hline $\mathrm{H}$ & -6.21487900 & 2.23117500 & 0.04813600 \\
\hline $\mathrm{H}$ & -5.75418100 & 0.85750000 & -1.00249300 \\
\hline $\mathrm{C}$ & 0.46460800 & 3.01812100 & 0.51888400 \\
\hline $\mathrm{H}$ & -0.35042800 & 3.25186800 & 1.20211500 \\
\hline $\mathrm{H}$ & 1.40333100 & 3.20096900 & 1.03674400 \\
\hline C & 0.39565000 & 3.83711200 & -0.77083000 \\
\hline $\mathrm{H}$ & 1.24854300 & 3.61298500 & -1.41564800 \\
\hline $\mathrm{H}$ & -0.52672100 & 3.64991400 & -1.32720400 \\
\hline $\mathrm{H}$ & 0.42901600 & 4.89898500 & -0.51092000 \\
\hline $\mathrm{C}$ & 5.32244100 & -1.06018100 & -0.08011000 \\
\hline
\end{tabular}




$\begin{array}{lrrr}\mathrm{H} & 5.36545500 & -1.75648100 & -0.91905500 \\ \mathrm{H} & 6.07830500 & -0.28782600 & -0.18516300 \\ \mathrm{H} & 5.47422900 & -1.61528100 & 0.84861900 \\ \mathrm{C} & 0.37140000 & -2.66730200 & -0.51093200 \\ \mathrm{H} & 1.29504300 & -2.90125600 & -1.02516500 \\ \mathrm{H} & -0.44745700 & -2.78795600 & -1.21641200 \\ \mathrm{C} & 0.21260000 & -3.53722300 & 0.73378000 \\ \mathrm{H} & -0.69904900 & -3.31033000 & 1.29155000 \\ \mathrm{H} & 0.16386100 & -4.58027400 & 0.40804600 \\ \mathrm{H} & 1.07362200 & -3.42443500 & 1.39444100\end{array}$

Table S5i. Cartesian coordinates for 8AB.

\begin{tabular}{|c|c|c|c|}
\hline \multirow[t]{2}{*}{ Atom } & \multicolumn{3}{|c|}{ Coordinates (Angstroms) } \\
\hline & $X$ & $\mathrm{Y}$ & $\mathrm{Z}$ \\
\hline $\mathrm{C}$ & 1.25889300 & -1.10535300 & 0.21794600 \\
\hline $\mathrm{C}$ & 0.01892700 & -0.45169600 & 0.09160900 \\
\hline $\mathrm{C}$ & -0.00745200 & 0.94767600 & -0.19555700 \\
\hline $\mathrm{C}$ & 1.21446200 & 1.62549600 & -0.31293500 \\
\hline C & -2.40027700 & 0.92859000 & -0.13171700 \\
\hline $\mathrm{C}$ & -2.34941700 & -0.50200700 & 0.07462900 \\
\hline C & -3.67114800 & -1.21159200 & 0.07568200 \\
\hline $\mathrm{C}$ & -4.72628400 & 1.02400000 & 0.00707800 \\
\hline $\mathrm{H}$ & 1.31418600 & -2.16663900 & 0.38267600 \\
\hline $\mathrm{H}$ & 1.22530700 & 2.69037500 & -0.49169100 \\
\hline $\mathrm{N}$ & -4.77393700 & -0.39233900 & 0.11167100 \\
\hline $\mathrm{N}$ & -3.50815100 & 1.62553700 & -0.14421300 \\
\hline $\mathrm{N}$ & -1.18208800 & -1.13656900 & 0.22566800 \\
\hline $\mathrm{N}$ & -1.21057400 & 1.59733700 & -0.31868600 \\
\hline O & -3.78577400 & -2.43380800 & 0.03055000 \\
\hline O & -5.77019200 & 1.66938700 & 0.04102000 \\
\hline $\mathrm{N}$ & 3.57512900 & 1.76416100 & -0.44702900 \\
\hline $\mathrm{H}$ & 3.39053300 & 2.56377000 & -1.04207800 \\
\hline $\mathrm{N}$ & 3.68177600 & -1.11347500 & 0.12697000 \\
\hline $\mathrm{H}$ & 4.46406600 & -0.54817600 & 0.46525000 \\
\hline C & 2.43676600 & 0.97695800 & -0.19381000 \\
\hline C & 2.46414600 & -0.43699500 & 0.06801200 \\
\hline C & 4.80044000 & 1.80529600 & 0.19230900 \\
\hline C & 3.90974700 & -2.45150400 & -0.12330400 \\
\hline C & 5.34340100 & -2.89557100 & 0.06228900 \\
\hline $\mathrm{H}$ & 5.37477800 & -3.64774900 & 0.85630700 \\
\hline $\mathrm{H}$ & 5.68276200 & -3.37373200 & -0.86056800 \\
\hline $\mathrm{H}$ & 6.02261500 & -2.07998100 & 0.31690000 \\
\hline $\mathrm{C}$ & 5.68928800 & 2.95249700 & -0.21898500 \\
\hline $\mathrm{H}$ & 6.72980100 & 2.65752200 & -0.08054400 \\
\hline $\mathrm{H}$ & 5.52692400 & 3.26310400 & -1.25345700 \\
\hline
\end{tabular}




$\begin{array}{lrrr}\mathrm{H} & 5.48407300 & 3.80799700 & 0.43447500 \\ \mathrm{O} & 5.13216100 & 0.98467300 & 1.05035200 \\ \mathrm{O} & 3.02551100 & -3.23171800 & -0.47843900 \\ \mathrm{C} & -6.08232200 & -1.06453000 & 0.16222000 \\ \mathrm{H} & -6.83896200 & -0.30384000 & 0.32874200 \\ \mathrm{H} & -6.27064700 & -1.58476500 & -0.78010500 \\ \mathrm{H} & -6.08073100 & -1.79138800 & 0.97528400 \\ \mathrm{C} & -1.27584200 & 3.05408000 & -0.62176700 \\ \mathrm{H} & -0.46905700 & 3.27616000 & -1.31859900 \\ \mathrm{H} & -2.22003200 & 3.20705500 & -1.13995300 \\ \mathrm{C} & -1.21021500 & 3.91375800 & 0.64125300 \\ \mathrm{H} & -2.05505900 & 3.69741000 & 1.29903900 \\ \mathrm{H} & -0.28135700 & 3.75611100 & 1.19601900 \\ \mathrm{H} & -1.26060300 & 4.96620500 & 0.34828500 \\ \mathrm{C} & -1.11263800 & -2.60117000 & 0.59748800 \\ \mathrm{H} & -2.03870900 & -2.83842300 & 1.10561900 \\ \mathrm{H} & -0.30031500 & -2.68172600 & 1.31566600 \\ \mathrm{C} & -0.91362900 & -3.50108800 & -0.61969000 \\ \mathrm{H} & -0.86172700 & -4.53326600 & -0.26144900 \\ \mathrm{H} & -1.75907100 & -3.41822900 & -1.30449000 \\ \mathrm{H} & 0.01141500 & -3.27969500 & -1.15636600 \\ ---------1500\end{array}$

Table S5j. Cartesian coordinates for 9AB.

\begin{tabular}{|c|c|c|c|}
\hline \multirow[t]{2}{*}{ Atom } & \multicolumn{3}{|c|}{ Coordinates (Angstroms) } \\
\hline & $X$ & $\mathrm{Y}$ & $\mathrm{Z}$ \\
\hline C & -3.18833800 & -0.75318500 & -0.08556000 \\
\hline $\mathrm{C}$ & -1.95227100 & -1.36710100 & -0.15176900 \\
\hline $\mathrm{C}$ & -0.74354000 & -0.63798800 & -0.06988500 \\
\hline $\mathrm{C}$ & -0.80382800 & 0.78213800 & 0.08416200 \\
\hline C & -2.06789300 & 1.39693400 & 0.14497900 \\
\hline C & -3.24660400 & 0.67007100 & 0.07009900 \\
\hline $\mathrm{C}$ & 1.58955800 & 0.89599200 & 0.03560000 \\
\hline C & 1.62312600 & -0.54950100 & -0.01058000 \\
\hline $\mathrm{C}$ & 2.98206800 & -1.17728800 & 0.09696300 \\
\hline C & 3.90824600 & 1.10716600 & -0.10088500 \\
\hline $\mathrm{H}$ & -1.93326900 & -2.44177800 & -0.25350700 \\
\hline $\mathrm{H}$ & -2.13844000 & 2.47099600 & 0.24056800 \\
\hline $\mathrm{N}$ & 4.03818000 & -0.30605700 & -0.01409500 \\
\hline $\mathrm{N}$ & 2.65673400 & 1.65192100 & -0.02910200 \\
\hline $\mathrm{N}$ & 0.49736600 & -1.26112400 & -0.11246700 \\
\hline $\mathrm{N}$ & 0.36227000 & 1.50822700 & 0.14823500 \\
\hline 0 & 3.16220200 & -2.37702900 & 0.28974900 \\
\hline 0 & 4.91401700 & 1.80110200 & -0.21953400 \\
\hline C & -4.43422900 & -1.60140800 & -0.22564700 \\
\hline $\mathrm{H}$ & -4.22114900 & -2.60399900 & 0.15860800 \\
\hline
\end{tabular}




$\begin{array}{rrrr}\mathrm{H} & -5.23964700 & -1.19738000 & 0.39344300 \\ \mathrm{C} & -4.55877300 & 1.40766400 & 0.20156200 \\ \mathrm{H} & -5.32711800 & 0.93043100 & -0.41206900 \\ \mathrm{H} & -4.43269300 & 2.42409000 & -0.18411100 \\ \mathrm{C} & -5.04328200 & 1.48357700 & 1.66607100 \\ \mathrm{H} & -5.19192000 & 0.48744100 & 2.09402200 \\ \mathrm{H} & -4.31783800 & 2.01533700 & 2.28922600 \\ \mathrm{H} & -5.99547400 & 2.02022200 & 1.71372900 \\ \mathrm{C} & -4.91337400 & -1.71591300 & -1.68762300 \\ \mathrm{H} & -5.81731100 & -2.33057800 & -1.73812300 \\ \mathrm{H} & -5.14530800 & -0.73535400 & -2.11507600 \\ \mathrm{H} & -4.14620600 & -2.18320700 & -2.31302300 \\ \mathrm{C} & 0.34253600 & 2.98910800 & 0.30462400 \\ \mathrm{H} & -0.47616800 & 3.23259900 & 0.97942800 \\ \mathrm{H} & 1.27646100 & 3.24804700 & 0.79848200 \\ \mathrm{C} & 0.22610500 & 3.71419700 & -1.03657500 \\ \mathrm{H} & 1.08006500 & 3.48198000 & -1.67714000 \\ \mathrm{H} & -0.69411200 & 3.45173700 & -1.56545300 \\ \mathrm{H} & 0.21822900 & 4.79165500 & -0.84882500 \\ \mathrm{C} & 5.38227000 & -0.90242400 & 0.04758100 \\ \mathrm{H} & 6.10215100 & -0.11469700 & -0.15247700 \\ \mathrm{H} & 5.55292400 & -1.33038000 & 1.03818600 \\ \mathrm{H} & 5.46052500 & -1.69367600 & -0.69941800 \\ \mathrm{C} & 0.52083000 & -2.75895300 & -0.32137500 \\ \mathrm{H} & -0.26477200 & -2.96491100 & -1.04384700 \\ \mathrm{H} & 1.47223800 & -2.99691100 & -0.77972100 \\ \mathrm{C} & 0.34052800 & -3.53016200 & 0.98419700 \\ \mathrm{H} & -0.60339200 & -3.29585400 & 1.48142800 \\ \mathrm{H} & 0.34516200 & -4.59676800 & 0.74220200 \\ \mathrm{H} & 1.16666100 & -3.32993800 & 1.66814300 \\ ----------------------------------------------------- \\ & & \end{array}$

Table S5k. Cartesian coordinates for 1A.

\begin{tabular}{|c|c|c|c|}
\hline \multirow[t]{2}{*}{ Atom } & \multicolumn{3}{|c|}{ Coordinates (Angstroms) } \\
\hline & $X$ & $\mathrm{Y}$ & $\mathrm{Z}$ \\
\hline C & 2.82774100 & 1.35200600 & -0.23217300 \\
\hline C & 1.51492900 & 1.77940800 & -0.32338500 \\
\hline C & 0.42025100 & 0.90251000 & -0.16051800 \\
\hline C & 0.66176100 & -0.47364500 & 0.10429100 \\
\hline $\mathrm{C}$ & 1.99912800 & -0.91457400 & 0.18858800 \\
\hline $\mathrm{C}$ & 3.04612000 & -0.03220400 & 0.02709800 \\
\hline C & -1.68741000 & -0.91487400 & 0.09594700 \\
\hline C & -1.91214500 & 0.51223000 & -0.04942100 \\
\hline C & -3.34744000 & 0.95984100 & 0.04482100 \\
\hline $\mathrm{C}$ & -3.95921400 & -1.43290100 & -0.02692500 \\
\hline $\mathrm{H}$ & 1.34921100 & 2.82864400 & -0.51123700 \\
\hline
\end{tabular}




$\begin{array}{lrrr}\text { H } & 2.21502400 & -1.95603700 & 0.36572600 \\ \mathrm{~N} & -4.27640500 & -0.04642300 & -0.02873200 \\ \mathrm{~N} & -2.64433600 & -1.80248100 & 0.08348800 \\ \mathrm{~N} & -0.90118300 & 1.35650900 & -0.22640100 \\ \mathrm{~N} & -0.39042500 & -1.34593100 & 0.24930500 \\ \mathrm{O} & -3.67430700 & 2.13249800 & 0.19305100 \\ \mathrm{O} & -4.85946500 & -2.26008800 & -0.10429000 \\ \mathrm{C} & 4.45746900 & -0.58071600 & 0.13027600 \\ \mathrm{C} & 3.94856000 & 2.34557300 & -0.40724300 \\ \mathrm{C} & -0.17156400 & -2.79400500 & 0.52701200 \\ \mathrm{H} & 0.66845900 & -2.86842400 & 1.21603500 \\ \mathrm{H} & -1.06488000 & -3.13172500 & 1.04820300 \\ \mathrm{C} & 0.04523900 & -3.60747600 & -0.74942000 \\ \mathrm{H} & -0.82888700 & -3.54567100 & -1.40158600 \\ \mathrm{H} & 0.92540000 & -3.27326300 & -1.30524400 \\ \mathrm{H} & 0.19422500 & -4.65410900 & -0.46961300 \\ \mathrm{C} & -5.68934000 & 0.36746400 & 0.00220800 \\ \mathrm{H} & -5.85053300 & 1.14279300 & -0.74752400 \\ \mathrm{H} & -6.29555700 & -0.50707700 & -0.21349600 \\ \mathrm{H} & -5.93787400 & 0.76644200 & 0.98861100 \\ \mathrm{C} & -1.12458400 & 2.82069600 & -0.54028900 \\ \mathrm{H} & -0.38810700 & 3.06967900 & -1.30037700 \\ \mathrm{H} & -2.10778700 & 2.89949500 & -0.98664900 \\ \mathrm{C} & -1.01856900 & 3.70289600 & 0.70171800 \\ \mathrm{H} & -1.15080300 & 4.74007600 & 0.38099500 \\ \mathrm{H} & -1.80685900 & 3.45954800 & 1.41539100 \\ \mathrm{H} & -0.04797200 & 3.61760900 & 1.19508900 \\ \mathrm{H} & 4.58651600 & 2.38472800 & 0.48023500 \\ \mathrm{H} & 3.54616100 & 3.34424100 & -0.58258800 \\ \mathrm{H} & 4.58381900 & 2.07888100 & -1.25683400 \\ \mathrm{~F} & 5.14697100 & 0.00493800 & 1.14058200 \\ \mathrm{~F} & 4.48011100 & -1.91142600 & 0.36054500 \\ \mathrm{~F} & 5.16197200 & -0.36686800 & -1.00827800 \\ -----1900--1000 \\ \end{array}$

Table S5l. Cartesian coordinates for 1B.

\begin{tabular}{|c|c|c|c|}
\hline \multirow[t]{2}{*}{ Atom } & \multicolumn{3}{|c|}{ Coordinates (Angstroms) } \\
\hline & $X$ & $\mathrm{Y}$ & $\mathrm{Z}$ \\
\hline C & -3.01907500 & -0.08759500 & -0.04147100 \\
\hline $\mathrm{C}$ & -1.89968400 & -0.87771500 & -0.17432900 \\
\hline C & -0.60442500 & -0.32529000 & -0.06564700 \\
\hline C & -0.46996800 & 1.06995500 & 0.18806800 \\
\hline C & -1.63528800 & 1.85330400 & 0.30536600 \\
\hline C & -2.90719600 & 1.31537800 & 0.19874600 \\
\hline C & 1.91305800 & 0.85793200 & 0.11006300 \\
\hline C & 1.74519100 & -0.57760300 & -0.03505400 \\
\hline
\end{tabular}




\begin{tabular}{|c|c|c|c|}
\hline $\mathrm{C}$ & 3.00862600 & -1.39451400 & 0.00968000 \\
\hline $\mathrm{C}$ & 4.23710100 & 0.74651100 & -0.07198700 \\
\hline $\mathrm{H}$ & -2.03624800 & -1.93234100 & -0.34783500 \\
\hline $\mathrm{H}$ & -1.54946900 & 2.91655300 & 0.47216800 \\
\hline $\mathrm{N}$ & 4.17232900 & -0.67260600 & -0.07717000 \\
\hline $\mathrm{N}$ & 3.07115400 & 1.45556000 & 0.06885500 \\
\hline $\mathrm{N}$ & 0.53825400 & -1.11695400 & -0.17243100 \\
\hline $\mathrm{N}$ & 0.78371600 & 1.62434200 & 0.29190400 \\
\hline 0 & 3.01481800 & -2.61464900 & 0.13365300 \\
\hline O & 5.32203300 & 1.30562700 & -0.17374800 \\
\hline C & -4.10354100 & 2.21811300 & 0.33626300 \\
\hline C & -4.37698600 & -0.74606500 & -0.15961400 \\
\hline $\mathrm{F}$ & -5.12283300 & -0.57148700 & 0.96088500 \\
\hline $\mathrm{F}$ & -4.28775200 & -2.07905500 & -0.36526900 \\
\hline $\mathrm{F}$ & -5.09699600 & -0.23815400 & -1.19247800 \\
\hline C & 0.97135600 & 3.07886400 & 0.55635900 \\
\hline $\mathrm{H}$ & 0.20180300 & 3.37921400 & 1.26574500 \\
\hline $\mathrm{H}$ & 1.93706900 & 3.16741400 & 1.04936300 \\
\hline C & 0.94288800 & 3.91289000 & -0.72530100 \\
\hline $\mathrm{H}$ & 1.75295300 & 3.61903200 & -1.39667100 \\
\hline $\mathrm{H}$ & -0.00811100 & 3.81646600 & -1.25579600 \\
\hline $\mathrm{H}$ & 1.08085000 & 4.96407400 & -0.45715700 \\
\hline C & 5.42300400 & -1.45021300 & -0.08231900 \\
\hline $\mathrm{H}$ & 5.37094800 & -2.20822700 & -0.86484800 \\
\hline $\mathrm{H}$ & 6.24028700 & -0.76084700 & -0.27090900 \\
\hline $\mathrm{H}$ & 5.55656200 & -1.94211200 & 0.88389700 \\
\hline C & 0.34814100 & -2.58647700 & -0.48819600 \\
\hline $\mathrm{H}$ & -0.45929500 & -2.62476500 & -1.21541400 \\
\hline $\mathrm{H}$ & 1.25530000 & -2.92085500 & -0.97544000 \\
\hline C & 0.06423500 & -3.41659700 & 0.76150200 \\
\hline $\mathrm{H}$ & -0.09131800 & -4.45067200 & 0.44144800 \\
\hline $\mathrm{H}$ & 0.91598700 & -3.39124000 & 1.44245800 \\
\hline $\mathrm{H}$ & -0.83062400 & -3.08397000 & 1.29188400 \\
\hline $\mathrm{H}$ & -4.72366200 & 1.91708700 & 1.18610400 \\
\hline $\mathrm{H}$ & -3.78845600 & 3.25097300 & 0.48937800 \\
\hline $\mathrm{H}$ & -4.73393200 & 2.17698000 & -0.55673700 \\
\hline
\end{tabular}

Table S5m. Cartesian coordinates for 2A.

\begin{tabular}{lrrr} 
Atom & \multicolumn{3}{c}{ Coordinates (Angstroms) } \\
& X & Y & Z \\
\hline C & -1.84743800 & 1.67206100 & 0.35386500 \\
C & -0.72919100 & 0.83303300 & 0.16993700 \\
C & -0.92378300 & -0.54799900 & -0.11380400 \\
C & -2.24267800 & -1.02897700 & -0.19881800 \\
C & 1.43679000 & -0.91289700 & -0.10768800
\end{tabular}




\begin{tabular}{|c|c|c|c|}
\hline $\mathrm{C}$ & 1.61409900 & 0.52201900 & 0.04900800 \\
\hline C & 3.03488400 & 1.01850200 & -0.05316600 \\
\hline $\mathrm{C}$ & 3.72578900 & -1.35122500 & 0.02004900 \\
\hline $\mathrm{H}$ & -1.71157300 & 2.71841500 & 0.57729500 \\
\hline $\mathrm{H}$ & -2.45299600 & -2.07223700 & -0.37639700 \\
\hline $\mathrm{N}$ & 3.99713400 & 0.04495500 & 0.01922100 \\
\hline $\mathrm{N}$ & 2.42322200 & -1.76531900 & -0.09584000 \\
\hline $\mathrm{N}$ & 0.57950100 & 1.33055000 & 0.23987000 \\
\hline $\mathrm{N}$ & 0.15632300 & -1.38490000 & -0.26928300 \\
\hline O & 3.31737500 & 2.20111100 & -0.20767600 \\
\hline O & 4.65116800 & -2.14823300 & 0.10239600 \\
\hline C & -3.15426700 & 1.21982000 & 0.24299100 \\
\hline $\mathrm{C}$ & -3.30057000 & -0.16028900 & -0.04115400 \\
\hline $\mathrm{C}$ & -0.01735500 & -2.83636200 & -0.55867200 \\
\hline $\mathrm{H}$ & -0.85432600 & -2.93042500 & -1.24941100 \\
\hline $\mathrm{H}$ & 0.88618900 & -3.14192100 & -1.08239400 \\
\hline C & -0.20967400 & -3.66605600 & 0.71126400 \\
\hline $\mathrm{H}$ & 0.66163300 & -3.58198200 & 1.36465400 \\
\hline $\mathrm{H}$ & -1.10029800 & -3.36396600 & 1.26908300 \\
\hline $\mathrm{H}$ & -0.32577300 & -4.71458400 & 0.42326300 \\
\hline C & 0.75761400 & 2.79791900 & 0.57065300 \\
\hline $\mathrm{H}$ & 0.02148000 & 3.01243800 & 1.34163300 \\
\hline $\mathrm{H}$ & 1.74182000 & 2.90218800 & 1.00955800 \\
\hline $\mathrm{C}$ & 0.61066600 & 3.69208300 & -0.65822300 \\
\hline $\mathrm{H}$ & 1.39472900 & 3.47856100 & -1.38589500 \\
\hline $\mathrm{H}$ & -0.36455000 & 3.58895900 & -1.13888300 \\
\hline $\mathrm{H}$ & 0.72085300 & 4.72792200 & -0.32510900 \\
\hline C & 5.39576400 & 0.50547400 & -0.01738400 \\
\hline $\mathrm{H}$ & 6.03113300 & -0.34669400 & 0.20388900 \\
\hline $\mathrm{H}$ & 5.62901000 & 0.90470600 & -1.00738600 \\
\hline $\mathrm{H}$ & 5.53170300 & 1.29133900 & 0.72627400 \\
\hline $\mathrm{N}$ & -4.64302100 & -0.76488000 & -0.18325200 \\
\hline O & -5.52398100 & -0.08010600 & -0.69814100 \\
\hline O & -4.79035700 & -1.92277500 & 0.20573700 \\
\hline C & -4.29554600 & 2.17984500 & 0.45412200 \\
\hline $\mathrm{H}$ & -4.79286700 & 2.40671900 & -0.49259900 \\
\hline $\mathrm{H}$ & -5.05171800 & 1.76592300 & 1.12656100 \\
\hline $\mathrm{H}$ & -3.92083200 & 3.11011400 & 0.88445000 \\
\hline
\end{tabular}

Table S5n. Cartesian coordinates for 2B.

\begin{tabular}{|c|c|c|c|}
\hline \multirow[t]{2}{*}{ Atom } & \multicolumn{3}{|c|}{ Coordinates (Angstroms) } \\
\hline & $X$ & $\mathrm{Y}$ & $\mathrm{Z}$ \\
\hline C & 2.12820400 & -1.01014100 & 0.21535300 \\
\hline $\mathrm{C}$ & 0.86372600 & -0.41055300 & 0.09150200 \\
\hline $\mathrm{C}$ & 0.78954800 & 0.99204500 & -0.15532300 \\
\hline
\end{tabular}




\begin{tabular}{|c|c|c|c|}
\hline C & 1.98538700 & 1.72972000 & -0.24784100 \\
\hline C & -1.59923800 & 0.87354200 & -0.10368600 \\
\hline C & -1.49139400 & -0.57181400 & 0.03041900 \\
\hline C & -2.78873900 & -1.33670300 & -0.03294500 \\
\hline C & -3.92864700 & 0.85033000 & 0.06058600 \\
\hline $\mathrm{H}$ & 2.25014000 & -2.06574500 & 0.39798500 \\
\hline $\mathrm{H}$ & 1.94001000 & 2.79906000 & -0.38845800 \\
\hline N & -3.92219400 & -0.56929800 & 0.05468000 \\
\hline $\mathrm{N}$ & -2.73178900 & 1.51375700 & -0.06646900 \\
\hline $\mathrm{N}$ & -0.31270100 & -1.16094500 & 0.17664100 \\
\hline N & -0.43888800 & 1.59630200 & -0.27193600 \\
\hline 0 & -2.84057000 & -2.55333800 & -0.17100700 \\
\hline 0 & -4.98801800 & 1.45452000 & 0.15936900 \\
\hline C & 3.27158100 & -0.25275200 & 0.07941900 \\
\hline C & 3.24344600 & 1.15123400 & -0.14849400 \\
\hline $\mathrm{N}$ & 4.53859300 & -0.99645200 & 0.18547900 \\
\hline C & -0.56818000 & 3.05776500 & -0.53674700 \\
\hline $\mathrm{H}$ & 0.22626100 & 3.33014600 & -1.22970200 \\
\hline $\mathrm{H}$ & -1.51949200 & 3.18141800 & -1.05024400 \\
\hline C & -0.53689000 & 3.88965700 & 0.74615000 \\
\hline $\mathrm{H}$ & -1.37080700 & 3.62488100 & 1.40000100 \\
\hline $\mathrm{H}$ & 0.39871700 & 3.75959400 & 1.29640200 \\
\hline $\mathrm{H}$ & -0.63171600 & 4.94495100 & 0.47557100 \\
\hline $\mathrm{C}$ & -0.18069700 & -2.64108500 & 0.47337100 \\
\hline $\mathrm{H}$ & 0.61676300 & -2.71940200 & 1.20824800 \\
\hline $\mathrm{H}$ & -1.10478200 & -2.94748800 & 0.94697700 \\
\hline C & 0.08648000 & -3.46245100 & -0.78562300 \\
\hline $\mathrm{H}$ & -0.75707700 & -3.39935500 & -1.47433300 \\
\hline $\mathrm{H}$ & 0.99775100 & -3.15356000 & -1.30230200 \\
\hline $\mathrm{H}$ & 0.20392400 & -4.50518300 & -0.47777300 \\
\hline C & -5.20395700 & -1.29542900 & 0.04555200 \\
\hline $\mathrm{H}$ & -5.99305800 & -0.57664100 & 0.24391800 \\
\hline $\mathrm{H}$ & -5.35503100 & -1.76589600 & -0.92867600 \\
\hline $\mathrm{H}$ & -5.18359100 & -2.06679700 & 0.81624400 \\
\hline 0 & 5.54125800 & -0.50876700 & -0.33526700 \\
\hline 0 & 4.52287800 & -2.07784400 & 0.77784600 \\
\hline C & 4.45607200 & 2.03438500 & -0.24790700 \\
\hline $\mathrm{H}$ & 5.15419800 & 1.85060200 & 0.57262000 \\
\hline $\mathrm{H}$ & 4.99936200 & 1.84062500 & -1.17685300 \\
\hline $\mathrm{H}$ & 4.15268400 & 3.08216000 & -0.22863200 \\
\hline
\end{tabular}

Table S5o. Cartesian coordinates for $3 \mathrm{~A}$.

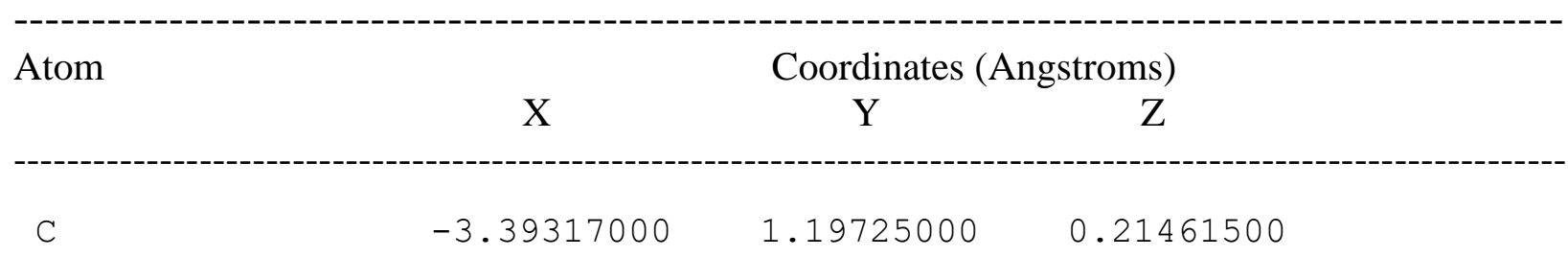




\begin{tabular}{|c|c|c|c|}
\hline C & -2.09704300 & 1.67428700 & 0.31087800 \\
\hline C & -0.97440100 & 0.83413400 & 0.14505500 \\
\hline C & -1.16740700 & -0.55265700 & -0.12574600 \\
\hline $\mathrm{C}$ & -2.48463900 & -1.03460100 & -0.21988100 \\
\hline $\mathrm{C}$ & -3.57019700 & -0.19106300 & -0.05637100 \\
\hline $\mathrm{C}$ & 1.19699600 & -0.91212200 & -0.09189900 \\
\hline C & 1.37142800 & 0.52272200 & 0.04558400 \\
\hline C & 2.79046000 & 1.01906000 & -0.05108500 \\
\hline C & 3.48364500 & -1.34986800 & 0.06152500 \\
\hline $\mathrm{H}$ & -1.96605700 & 2.72711600 & 0.50591500 \\
\hline $\mathrm{H}$ & -2.68808000 & -2.07976800 & -0.40474500 \\
\hline $\mathrm{N}$ & 3.75363800 & 0.04704300 & 0.03770000 \\
\hline $\mathrm{N}$ & 2.18454400 & -1.76627700 & -0.05954100 \\
\hline $\mathrm{N}$ & 0.33082900 & 1.33284800 & 0.21385100 \\
\hline $\mathrm{N}$ & -0.08077900 & -1.38652600 & -0.26173900 \\
\hline 0 & 3.07659700 & 2.20040000 & -0.21531600 \\
\hline O & 4.41121500 & -2.14345600 & 0.16690400 \\
\hline C & -0.24887800 & -2.84012200 & -0.54160900 \\
\hline $\mathrm{H}$ & -1.07896300 & -2.94050500 & -1.23939900 \\
\hline $\mathrm{H}$ & 0.66091600 & -3.14922500 & -1.05216300 \\
\hline C & -0.45547800 & -3.66026800 & 0.73222100 \\
\hline $\mathrm{H}$ & 0.40831200 & -3.57146100 & 1.39501800 \\
\hline $\mathrm{H}$ & -1.35235000 & -3.35256100 & 1.27666400 \\
\hline $\mathrm{H}$ & -0.56897100 & -4.71113500 & 0.45152200 \\
\hline C & 5.15129000 & 0.50939200 & 0.00285700 \\
\hline $\mathrm{H}$ & 5.28915500 & 1.28923300 & 0.75279700 \\
\hline $\mathrm{H}$ & 5.78781200 & -0.34403200 & 0.21581700 \\
\hline $\mathrm{H}$ & 5.38218300 & 0.91769000 & -0.98394000 \\
\hline C & 0.50460900 & 2.80553700 & 0.51811400 \\
\hline $\mathrm{H}$ & 1.48548400 & 2.92094200 & 0.96139100 \\
\hline $\mathrm{H}$ & -0.23820900 & 3.03489200 & 1.27820800 \\
\hline C & 0.36622400 & 3.67538500 & -0.72924300 \\
\hline $\mathrm{H}$ & 0.46856500 & 4.71840000 & -0.41654900 \\
\hline $\mathrm{H}$ & 1.15828800 & 3.45107400 & -1.44507200 \\
\hline $\mathrm{H}$ & -0.60369300 & 3.55762800 & -1.21724700 \\
\hline C & -4.93354200 & -0.79693500 & -0.16606600 \\
\hline $\mathrm{H}$ & -5.78857900 & -0.11825600 & -0.02444200 \\
\hline 0 & -5.11627500 & -1.98041100 & -0.39916500 \\
\hline C & -4.55343900 & 2.14433100 & 0.39717200 \\
\hline $\mathrm{H}$ & -5.19273400 & 2.17320700 & -0.49092000 \\
\hline $\mathrm{H}$ & -5.17904000 & 1.85052900 & 1.24644300 \\
\hline $\mathrm{H}$ & -4.19270500 & 3.15757100 & 0.58269100 \\
\hline
\end{tabular}

Table S5p. Cartesian coordinates for 3B.

\begin{tabular}{|c|c|c|c|}
\hline \multirow[t]{2}{*}{ Atom } & \multicolumn{3}{|c|}{ Coordinates (Angstroms) } \\
\hline & $X$ & $\mathrm{Y}$ & $\mathrm{Z}$ \\
\hline
\end{tabular}




\begin{tabular}{|c|c|c|c|}
\hline C & 3.52968100 & -0.35273600 & 0.08535000 \\
\hline C & 2.35615900 & -1.07500700 & 0.20557100 \\
\hline $\mathrm{C}$ & 1.10024500 & -0.45253300 & 0.08902700 \\
\hline $\mathrm{C}$ & 1.05001000 & 0.95398700 & -0.16181200 \\
\hline C & 2.25801700 & 1.67167900 & -0.26479700 \\
\hline C & 3.49403000 & 1.05583700 & -0.14838000 \\
\hline C & -1.34279300 & 0.87741700 & -0.10863000 \\
\hline $\mathrm{C}$ & -1.26172800 & -0.56607500 & 0.03830100 \\
\hline C & -2.57164400 & -1.30625500 & -0.00821800 \\
\hline C & -3.67202000 & 0.90366300 & 0.04859300 \\
\hline $\mathrm{H}$ & 2.45625900 & -2.13701900 & 0.37656100 \\
\hline $\mathrm{H}$ & 2.23341200 & 2.73898100 & -0.42710300 \\
\hline $\mathrm{N}$ & -3.69125300 & -0.51590000 & 0.06599500 \\
\hline $\mathrm{N}$ & -2.46424800 & 1.54169200 & -0.08201400 \\
\hline $\mathrm{N}$ & -0.09019500 & -1.17518500 & 0.18330000 \\
\hline $\mathrm{N}$ & -0.16915700 & 1.57766600 & -0.27818400 \\
\hline O & -2.65107000 & -2.52475800 & -0.12201500 \\
\hline O & -4.72267900 & 1.52758700 & 0.13273100 \\
\hline C & 4.81247300 & -1.09871000 & 0.20718400 \\
\hline $\mathrm{H}$ & 5.73746700 & -0.50977100 & 0.10734800 \\
\hline O & 4.86867400 & -2.30312400 & 0.40619400 \\
\hline C & -0.27219800 & 3.04002000 & -0.54790600 \\
\hline $\mathrm{H}$ & 0.52559500 & 3.29597200 & -1.24307300 \\
\hline $\mathrm{H}$ & -1.22234300 & 3.18026900 & -1.05900500 \\
\hline C & -0.22186900 & 3.87485700 & 0.73232000 \\
\hline $\mathrm{H}$ & -1.05798300 & 3.62594600 & 1.38971400 \\
\hline $\mathrm{H}$ & 0.71342000 & 3.72947400 & 1.27930000 \\
\hline $\mathrm{H}$ & -0.29927000 & 4.93119100 & 0.46003100 \\
\hline $\mathrm{C}$ & -4.98565800 & -1.21841100 & 0.07161700 \\
\hline $\mathrm{H}$ & -4.98051200 & -1.97305400 & 0.85905100 \\
\hline $\mathrm{H}$ & -5.76156800 & -0.48100100 & 0.25302100 \\
\hline $\mathrm{H}$ & -5.14517000 & -1.70792600 & -0.89185900 \\
\hline $\mathrm{C}$ & 0.01360100 & -2.65433300 & 0.49109700 \\
\hline $\mathrm{H}$ & -0.91152200 & -2.93755800 & 0.97698000 \\
\hline $\mathrm{H}$ & 0.81874700 & -2.74424100 & 1.21575300 \\
\hline $\mathrm{C}$ & 0.24963200 & -3.49182300 & -0.76338700 \\
\hline $\mathrm{H}$ & 0.33819400 & -4.53576900 & -0.44974800 \\
\hline $\mathrm{H}$ & -0.59508700 & -3.40927300 & -1.44884300 \\
\hline $\mathrm{H}$ & 1.16713900 & -3.21267300 & -1.28620000 \\
\hline C & 4.74769500 & 1.88208500 & -0.26406900 \\
\hline $\mathrm{H}$ & 5.34498400 & 1.82538000 & 0.65196700 \\
\hline $\mathrm{H}$ & 5.37795600 & 1.53368600 & -1.08913400 \\
\hline $\mathrm{H}$ & 4.50304800 & 2.92986600 & -0.44486900 \\
\hline
\end{tabular}

Table S5q. Cartesian coordinates for 4A. 


\begin{tabular}{|c|c|c|c|}
\hline \multirow[t]{2}{*}{ Atom } & \multicolumn{3}{|c|}{ Coordinates (Angstroms) } \\
\hline & $\mathrm{X}$ & $\mathrm{Y}$ & $\mathrm{Z}$ \\
\hline C & 2.45405600 & -1.35406600 & 0.24854800 \\
\hline $\mathrm{C}$ & 1.24623500 & -0.62562300 & 0.11307200 \\
\hline $\mathrm{C}$ & 1.29720100 & 0.77924700 & -0.15397800 \\
\hline $\mathrm{C}$ & 2.55488100 & 1.40191500 & -0.26117600 \\
\hline $\mathrm{C}$ & -1.09435600 & 0.88643400 & -0.11991700 \\
\hline C & -1.12080900 & -0.55197100 & 0.05187800 \\
\hline C & -2.47971300 & -1.19230100 & 0.01088000 \\
\hline $\mathrm{C}$ & -3.41438700 & 1.09622200 & 0.00004400 \\
\hline $\mathrm{H}$ & 2.43527600 & -2.41715100 & 0.43103500 \\
\hline $\mathrm{H}$ & 2.66360700 & 2.46140800 & -0.43750700 \\
\hline $\mathrm{N}$ & -3.53809500 & -0.31834100 & 0.05472000 \\
\hline $\mathrm{N}$ & -2.16319400 & 1.63799700 & -0.12352200 \\
\hline $\mathrm{N}$ & 0.00577200 & -1.24699200 & 0.21332900 \\
\hline $\mathrm{N}$ & 0.13117100 & 1.49346800 & -0.27943800 \\
\hline 0 & -2.65389800 & -2.40438900 & -0.07403300 \\
\hline O & -4.42076300 & 1.79533100 & 0.04905400 \\
\hline $\mathrm{C}$ & 3.69087700 & -0.75267500 & 0.13477700 \\
\hline $\mathrm{C}$ & 3.68760200 & 0.63862700 & -0.11778500 \\
\hline $\mathrm{F}$ & 4.87783600 & 1.25561700 & -0.21978200 \\
\hline $\mathrm{C}$ & 0.14773400 & 2.95862100 & -0.55041900 \\
\hline $\mathrm{H}$ & 0.97210500 & 3.15150100 & -1.23505100 \\
\hline $\mathrm{H}$ & -0.78169000 & 3.17343700 & -1.07338400 \\
\hline $\mathrm{C}$ & 0.24707500 & 3.78557700 & 0.73199800 \\
\hline $\mathrm{H}$ & -0.61269800 & 3.59869100 & 1.37935100 \\
\hline $\mathrm{H}$ & 1.16281900 & 3.57057700 & 1.28931100 \\
\hline $\mathrm{H}$ & 0.25186800 & 4.84509800 & 0.46119100 \\
\hline C & -4.88076000 & -0.92219000 & 0.06045800 \\
\hline $\mathrm{H}$ & -4.93931600 & -1.65989400 & 0.86168400 \\
\hline $\mathrm{H}$ & -5.60079400 & -0.12532000 & 0.21945400 \\
\hline $\mathrm{H}$ & -5.06885800 & -1.41770500 & -0.89495000 \\
\hline C & -0.01216700 & -2.72187800 & 0.55714300 \\
\hline $\mathrm{H}$ & 0.77745300 & -2.85848100 & 1.29156100 \\
\hline $\mathrm{H}$ & -0.96011900 & -2.91691800 & 1.04229800 \\
\hline C & 0.16101000 & -3.60970600 & -0.67258900 \\
\hline $\mathrm{H}$ & 0.15887900 & -4.64877000 & -0.33109300 \\
\hline $\mathrm{H}$ & -0.66958600 & -3.47505900 & -1.36704800 \\
\hline $\mathrm{H}$ & 1.10141300 & -3.42367300 & -1.19619300 \\
\hline C & 4.98218300 & -1.50886200 & 0.26516700 \\
\hline $\mathrm{H}$ & 5.57514500 & -1.42458100 & -0.65136600 \\
\hline $\mathrm{H}$ & 5.58852300 & -1.10321000 & 1.08151900 \\
\hline $\mathrm{H}$ & 4.79319500 & -2.56518300 & 0.46283200 \\
\hline
\end{tabular}

Table S5r. Cartesian coordinates for 4B. 


\begin{tabular}{|c|c|c|c|}
\hline \multirow[t]{2}{*}{ Atom } & \multicolumn{3}{|c|}{ Coordinates (Angstroms) } \\
\hline & $\mathrm{X}$ & $\mathrm{Y}$ & $\mathrm{Z}$ \\
\hline $\mathrm{C}$ & 2.40177400 & -1.45076100 & 0.26480500 \\
\hline $\mathrm{C}$ & 1.22008700 & -0.68785800 & 0.12211100 \\
\hline $\mathrm{C}$ & 1.32368700 & 0.71280600 & -0.14690000 \\
\hline $\mathrm{C}$ & 2.60423700 & 1.29981800 & -0.24940400 \\
\hline $\mathrm{C}$ & -1.06637300 & 0.89643000 & -0.11589700 \\
\hline $\mathrm{C}$ & -1.14091600 & -0.53910400 & 0.05819000 \\
\hline $\mathrm{C}$ & -2.51946300 & -1.13582000 & 0.01456200 \\
\hline C & -3.37851400 & 1.18271300 & -0.00109400 \\
\hline $\mathrm{H}$ & 2.39692100 & -2.51359400 & 0.44926900 \\
\hline $\mathrm{H}$ & 2.70000200 & 2.36070600 & -0.42755200 \\
\hline $\mathrm{N}$ & -3.54877900 & -0.22818600 & 0.04807300 \\
\hline $\mathrm{N}$ & -2.11082400 & 1.68303800 & -0.12077300 \\
\hline $\mathrm{N}$ & -0.03798600 & -1.27133700 & 0.21915300 \\
\hline $\mathrm{N}$ & 0.17871200 & 1.46187200 & -0.27805000 \\
\hline O & -2.73158200 & -2.34241000 & -0.06429100 \\
\hline 0 & -4.36248900 & 1.91330500 & 0.04857600 \\
\hline C & 3.61198900 & -0.82409900 & 0.14391400 \\
\hline C & 3.76467000 & 0.55921300 & -0.11011700 \\
\hline C & 0.23684900 & 2.92458200 & -0.55869800 \\
\hline $\mathrm{H}$ & 1.06469400 & 3.09032600 & -1.24588600 \\
\hline $\mathrm{H}$ & -0.68749100 & 3.16154500 & -1.08106800 \\
\hline C & 0.35992800 & 3.75627000 & 0.71854700 \\
\hline $\mathrm{H}$ & -0.50450900 & 3.59739500 & 1.36716900 \\
\hline $\mathrm{H}$ & 1.26939700 & 3.51880300 & 1.27705200 \\
\hline $\mathrm{H}$ & 0.39406500 & 4.81359400 & 0.44125100 \\
\hline $\mathrm{C}$ & -4.90995700 & -0.78878000 & 0.04482600 \\
\hline $\mathrm{H}$ & -5.00785500 & -1.50240800 & 0.86413100 \\
\hline $\mathrm{H}$ & -5.60643800 & 0.03466900 & 0.16927500 \\
\hline $\mathrm{H}$ & -5.09555900 & -1.30390600 & -0.90048200 \\
\hline C & -0.09731500 & -2.74687500 & 0.55095500 \\
\hline $\mathrm{H}$ & 0.69013100 & -2.91226700 & 1.28206900 \\
\hline $\mathrm{H}$ & -1.04923200 & -2.92046900 & 1.03658100 \\
\hline $\mathrm{C}$ & 0.04938000 & -3.62556400 & -0.68896100 \\
\hline $\mathrm{H}$ & 0.02981300 & -4.66807300 & -0.35888900 \\
\hline $\mathrm{H}$ & -0.78349100 & -3.46651000 & -1.37550500 \\
\hline $\mathrm{H}$ & 0.98929800 & -3.45158000 & -1.21770600 \\
\hline C & 5.13015300 & 1.16992600 & -0.21211200 \\
\hline $\mathrm{H}$ & 5.68999800 & 1.01751600 & 0.71679400 \\
\hline $\mathrm{H}$ & 5.70479400 & 0.69397800 & -1.01367200 \\
\hline $\mathrm{H}$ & 5.06490500 & 2.23994700 & -0.41280200 \\
\hline $\mathrm{F}$ & 4.73155400 & -1.56577000 & 0.27427900 \\
\hline
\end{tabular}

Table S5s. Cartesian coordinates for 5A. 


\begin{tabular}{|c|c|c|c|}
\hline \multirow[t]{2}{*}{ Atom } & \multicolumn{3}{|c|}{ Coordinates (Angstroms) } \\
\hline & $\mathrm{X}$ & $\mathrm{Y}$ & $\mathrm{Z}$ \\
\hline $\mathrm{C}$ & -2.08648500 & -1.57924000 & -0.27942300 \\
\hline $\mathrm{C}$ & -0.93415100 & -0.77228600 & -0.13432200 \\
\hline C & -1.08807600 & 0.62239900 & 0.13032400 \\
\hline C & -2.38870600 & 1.15156600 & 0.22507400 \\
\hline C & 1.28916800 & 0.90353800 & 0.10747200 \\
\hline C & 1.42069900 & -0.53055400 & -0.05616400 \\
\hline $\mathrm{C}$ & 2.82229700 & -1.07074000 & 0.00422600 \\
\hline C & 3.58867700 & 1.27947300 & -0.00745100 \\
\hline $\mathrm{H}$ & -1.99225500 & -2.63836000 & -0.46128900 \\
\hline $\mathrm{H}$ & -2.54968500 & 2.20442000 & 0.39807900 \\
\hline $\mathrm{N}$ & 3.81524500 & -0.12345400 & -0.03821600 \\
\hline $\mathrm{N}$ & 2.30118200 & 1.73014800 & 0.10715400 \\
\hline $\mathrm{N}$ & 0.34891700 & -1.30609500 & -0.22057500 \\
\hline $\mathrm{N}$ & 0.02324300 & 1.42006800 & 0.26461600 \\
\hline O & 3.08186300 & -2.26632700 & 0.10319900 \\
\hline O & 4.54146900 & 2.04913600 & -0.06648600 \\
\hline C & -3.36685200 & -1.06916500 & -0.17716000 \\
\hline C & -3.48451600 & 0.32556900 & 0.07593800 \\
\hline C & -0.09830600 & 2.87955000 & 0.53817000 \\
\hline $\mathrm{H}$ & -0.93319700 & 3.01223000 & 1.22462900 \\
\hline $\mathrm{H}$ & 0.81391000 & 3.15917000 & 1.06079100 \\
\hline $\mathrm{C}$ & -0.25651700 & 3.69982900 & -0.74257400 \\
\hline $\mathrm{H}$ & 0.61652100 & 3.57967800 & -1.38806300 \\
\hline $\mathrm{H}$ & -1.15186000 & 3.41788200 & -1.30337400 \\
\hline $\mathrm{H}$ & -0.34153700 & 4.75538800 & -0.46952000 \\
\hline C & 5.19768700 & -0.62953400 & -0.02301400 \\
\hline $\mathrm{H}$ & 5.32486300 & -1.35378800 & -0.82880400 \\
\hline $\mathrm{H}$ & 5.86154000 & 0.21818900 & -0.16228600 \\
\hline $\mathrm{H}$ & 5.40203300 & -1.11910800 & 0.93198100 \\
\hline $\mathrm{C}$ & 0.47365600 & -2.77988900 & -0.54315100 \\
\hline $\mathrm{H}$ & -0.29700500 & -2.98348700 & -1.28219300 \\
\hline $\mathrm{H}$ & 1.43777700 & -2.91606900 & -1.01631800 \\
\hline C & 0.34966300 & -3.65647300 & 0.70077400 \\
\hline $\mathrm{H}$ & 0.41587100 & -4.69938400 & 0.37808700 \\
\hline $\mathrm{H}$ & 1.16743800 & -3.45876900 & 1.39527100 \\
\hline $\mathrm{H}$ & -0.60249500 & -3.51738300 & 1.21768100 \\
\hline C & -4.56944300 & -1.95712200 & -0.32884800 \\
\hline $\mathrm{H}$ & -5.19506500 & -1.92403400 & 0.56890900 \\
\hline $\mathrm{H}$ & -5.19182600 & -1.62972500 & -1.16832500 \\
\hline $\mathrm{H}$ & -4.26687500 & -2.99074900 & -0.50411300 \\
\hline $\mathrm{Cl}$ & -5.06692400 & 1.03971900 & 0.19912100 \\
\hline
\end{tabular}

Table S5t. Cartesian coordinates for 5B. 


\begin{tabular}{|c|c|c|c|}
\hline \multirow[t]{2}{*}{ Atom } & \multicolumn{3}{|c|}{ Coordinates (Angstroms) } \\
\hline & $\mathrm{X}$ & $\mathrm{Y}$ & $\mathrm{Z}$ \\
\hline C & -2.26535800 & -1.16222800 & -0.21722900 \\
\hline $\mathrm{C}$ & -1.02054700 & -0.50590700 & -0.09360600 \\
\hline $\mathrm{C}$ & -0.99924100 & 0.89888300 & 0.16274900 \\
\hline $\mathrm{C}$ & -2.22351900 & 1.58945200 & 0.26688400 \\
\hline $\mathrm{C}$ & 1.39559300 & 0.87570700 & 0.11443300 \\
\hline $\mathrm{C}$ & 1.34456400 & -0.56381500 & -0.04533100 \\
\hline C & 2.66778500 & -1.27604400 & -0.00697300 \\
\hline $\mathrm{C}$ & 3.72272000 & 0.95926500 & -0.02723500 \\
\hline $\mathrm{H}$ & -2.32828800 & -2.22458100 & -0.39050600 \\
\hline $\mathrm{H}$ & -2.22612200 & 2.65615000 & 0.43421700 \\
\hline $\mathrm{N}$ & 3.77054800 & -0.46069900 & -0.06716800 \\
\hline $\mathrm{N}$ & 2.50371900 & 1.56823600 & 0.10234600 \\
\hline $\mathrm{N}$ & 0.18225800 & -1.19886600 & -0.19351300 \\
\hline $\mathrm{N}$ & 0.20600800 & 1.54834900 & 0.28159100 \\
\hline O & 2.77578700 & -2.49492500 & 0.08915700 \\
\hline 0 & 4.76452800 & 1.60272700 & -0.09381700 \\
\hline $\mathrm{Cl}$ & -4.94750400 & -1.31128100 & -0.24288500 \\
\hline $\mathrm{C}$ & -3.43616200 & -0.45054100 & -0.09501700 \\
\hline $\mathrm{C}$ & -3.44773100 & 0.95255500 & 0.14559200 \\
\hline C & 0.27492000 & 3.01144000 & 0.55658300 \\
\hline $\mathrm{H}$ & -0.53181600 & 3.24853800 & 1.24803900 \\
\hline $\mathrm{H}$ & 1.21940900 & 3.17075500 & 1.07247800 \\
\hline $\mathrm{C}$ & 0.21329000 & 3.84783600 & -0.72213300 \\
\hline $\mathrm{H}$ & 1.05719900 & 3.61684900 & -1.37607100 \\
\hline $\mathrm{H}$ & -0.71653100 & 3.68461600 & -1.27347300 \\
\hline $\mathrm{H}$ & 0.26816400 & 4.90496700 & -0.44748800 \\
\hline C & 5.07894500 & -1.13559100 & -0.07933600 \\
\hline $\mathrm{H}$ & 5.08808300 & -1.88590500 & -0.87085900 \\
\hline $\mathrm{H}$ & 5.83880000 & -0.38086200 & -0.25760600 \\
\hline $\mathrm{H}$ & 5.25165400 & -1.62765200 & 0.88074600 \\
\hline C & 0.11350700 & -2.67611400 & -0.51660300 \\
\hline $\mathrm{H}$ & -0.68736000 & -2.77795800 & -1.24479600 \\
\hline $\mathrm{H}$ & 1.04547700 & -2.93289300 & -1.00383200 \\
\hline C & -0.10341900 & -3.53334700 & 0.72794800 \\
\hline $\mathrm{H}$ & -0.16767100 & -4.57564100 & 0.40291800 \\
\hline $\mathrm{H}$ & 0.73948200 & -3.43892900 & 1.41415000 \\
\hline $\mathrm{H}$ & -1.02640700 & -3.28241900 & 1.25562200 \\
\hline C & -4.72879300 & 1.72397200 & 0.26101200 \\
\hline $\mathrm{H}$ & -5.32447100 & 1.62279200 & -0.65229800 \\
\hline $\mathrm{H}$ & -5.33888300 & 1.33691500 & 1.08412300 \\
\hline $\mathrm{H}$ & -4.53271900 & 2.78234600 & 0.43773600 \\
\hline
\end{tabular}


Table S5u. Cartesian coordinates for 6A.

\begin{tabular}{|c|c|c|c|}
\hline \multirow[t]{2}{*}{ Atom } & \multicolumn{3}{|c|}{ Coordinates (Angstroms) } \\
\hline & $X$ & $\mathrm{Y}$ & $\mathrm{Z}$ \\
\hline C & -2.44755500 & -1.37476300 & -0.21483400 \\
\hline $\mathrm{C}$ & -1.22943700 & -0.64349400 & -0.10406200 \\
\hline $\mathrm{C}$ & -1.30300200 & 0.77569400 & 0.14049700 \\
\hline C & -2.54817000 & 1.38659600 & 0.23137300 \\
\hline $\mathrm{C}$ & 1.09957000 & 0.88004700 & 0.11661600 \\
\hline C & 1.13886300 & -0.54705800 & -0.06469800 \\
\hline C & 2.48636300 & -1.17748300 & -0.05116200 \\
\hline C & 3.41810100 & 1.11313700 & 0.04164000 \\
\hline $\mathrm{H}$ & -2.42410600 & -2.44261800 & -0.36788200 \\
\hline $\mathrm{H}$ & -2.62970000 & 2.45302600 & 0.38088000 \\
\hline $\mathrm{N}$ & 3.54757000 & -0.29605100 & -0.04279100 \\
\hline $\mathrm{N}$ & 2.16533400 & 1.64536000 & 0.14873900 \\
\hline $\mathrm{N}$ & -0.00683900 & -1.24975200 & -0.21056700 \\
\hline $\mathrm{N}$ & -0.12983400 & 1.48909100 & 0.25944100 \\
\hline O & 2.68616000 & -2.39299100 & -0.03145200 \\
\hline O & 4.42627400 & 1.81941500 & 0.02887500 \\
\hline C & -3.67775300 & -0.78339000 & -0.11014600 \\
\hline C & -3.73586000 & 0.64616900 & 0.11027900 \\
\hline $\mathrm{C}$ & 4.89031700 & -0.89574100 & -0.05644900 \\
\hline $\mathrm{H}$ & 5.61223300 & -0.08594800 & -0.10150000 \\
\hline $\mathrm{H}$ & 5.04118500 & -1.48910900 & 0.84819000 \\
\hline $\mathrm{H}$ & 4.99095000 & -1.54665500 & -0.92665500 \\
\hline $\mathrm{C}$ & -0.15524100 & 2.95193100 & 0.52369100 \\
\hline $\mathrm{H}$ & -0.97838300 & 3.14173600 & 1.21172700 \\
\hline $\mathrm{H}$ & 0.77402000 & 3.18001300 & 1.04035600 \\
\hline $\mathrm{C}$ & -0.26933700 & 3.77712300 & -0.75906700 \\
\hline $\mathrm{H}$ & 0.59229300 & 3.60023000 & -1.40727100 \\
\hline $\mathrm{H}$ & -1.18182900 & 3.54842800 & -1.31635300 \\
\hline $\mathrm{H}$ & -0.28835800 & 4.83789000 & -0.49268000 \\
\hline C & 0.02138600 & -2.72364400 & -0.54918300 \\
\hline $\mathrm{H}$ & -0.78702600 & -2.88216400 & -1.25794300 \\
\hline $\mathrm{H}$ & 0.95828700 & -2.90690500 & -1.05991200 \\
\hline C & -0.09860700 & -3.60578200 & 0.69052500 \\
\hline $\mathrm{H}$ & 0.74961500 & -3.44960200 & 1.35905500 \\
\hline $\mathrm{H}$ & -1.02625800 & -3.42858500 & 1.24011500 \\
\hline $\mathrm{H}$ & -0.08875000 & -4.64894400 & 0.36146400 \\
\hline C & -4.94374100 & -1.58626700 & -0.21974900 \\
\hline $\mathrm{H}$ & -5.55247800 & -1.49676500 & 0.68794100 \\
\hline $\mathrm{H}$ & -5.55618200 & -1.24979000 & -1.06523100 \\
\hline $\mathrm{H}$ & -4.72074600 & -2.64363200 & -0.37069200 \\
\hline $\mathrm{N}$ & -4.92873100 & 1.25873300 & 0.19601900 \\
\hline $\mathrm{H}$ & -4.99603700 & 2.25520000 & 0.34699400 \\
\hline $\mathrm{H}$ & -5.79609700 & 0.75268800 & 0.10249300 \\
\hline
\end{tabular}


Table S5v. Cartesian coordinates for 6B.

\begin{tabular}{|c|c|c|c|}
\hline \multirow[t]{2}{*}{ Atom } & \multicolumn{3}{|c|}{ Coordinates (Angstroms) } \\
\hline & $\mathrm{X}$ & $\mathrm{Y}$ & $\mathrm{Z}$ \\
\hline $\mathrm{C}$ & -2.41201900 & -1.43199900 & -0.21702900 \\
\hline C & -1.22920000 & -0.68667300 & -0.10726500 \\
\hline $\mathrm{C}$ & -1.30555100 & 0.72824700 & 0.13713600 \\
\hline C & -2.58586600 & 1.32600000 & 0.22621000 \\
\hline C & 1.08627700 & 0.88112900 & 0.11334000 \\
\hline C & 1.14628400 & -0.53962900 & -0.06681700 \\
\hline C & 2.51140100 & -1.14510100 & -0.03790700 \\
\hline C & 3.39594300 & 1.16863300 & 0.04494900 \\
\hline $\mathrm{H}$ & -2.38296100 & -2.49972100 & -0.36772800 \\
\hline $\mathrm{H}$ & -2.67111100 & 2.39224500 & 0.37510100 \\
\hline $\mathrm{N}$ & 3.55123800 & -0.24388000 & -0.03251500 \\
\hline $\mathrm{N}$ & 2.13686700 & 1.67341600 & 0.14727700 \\
\hline $\mathrm{N}$ & 0.02400700 & -1.27090800 & -0.21987100 \\
\hline $\mathrm{N}$ & -0.16123500 & 1.46169700 & 0.25309300 \\
\hline O & 2.72555900 & -2.35710000 & -0.00092200 \\
\hline O & 4.39604300 & 1.88766800 & 0.03065700 \\
\hline C & -3.66403800 & -0.82822000 & -0.10414400 \\
\hline C & -3.74787700 & 0.59983700 & 0.10922000 \\
\hline $\mathrm{N}$ & -4.80054100 & -1.55885100 & -0.20461400 \\
\hline $\mathrm{H}$ & -4.76359900 & -2.56283300 & -0.30076500 \\
\hline $\mathrm{H}$ & -5.70906800 & -1.15187200 & -0.04975700 \\
\hline C & 4.90503800 & -0.81813400 & -0.02964400 \\
\hline $\mathrm{H}$ & 5.61168000 & 0.00397800 & -0.08948400 \\
\hline $\mathrm{H}$ & 5.06361200 & -1.38994900 & 0.88766600 \\
\hline $\mathrm{H}$ & 5.01922000 & -1.48549200 & -0.88561500 \\
\hline C & -0.20629500 & 2.92896200 & 0.51759900 \\
\hline $\mathrm{H}$ & -1.04126700 & 3.11308600 & 1.19075800 \\
\hline $\mathrm{H}$ & 0.71468700 & 3.16052100 & 1.04747300 \\
\hline C & -0.30308200 & 3.74541700 & -0.77133000 \\
\hline $\mathrm{H}$ & 0.56536200 & 3.56396900 & -1.40864400 \\
\hline $\mathrm{H}$ & -1.21077300 & 3.51462400 & -1.33565100 \\
\hline $\mathrm{H}$ & -0.32302800 & 4.80694800 & -0.50884500 \\
\hline C & 0.07401400 & -2.73380500 & -0.57602600 \\
\hline $\mathrm{H}$ & -0.72902900 & -2.88692200 & -1.29417800 \\
\hline $\mathrm{H}$ & 1.01632700 & -2.90426500 & -1.08155200 \\
\hline C & -0.05251400 & -3.64070600 & 0.64681000 \\
\hline $\mathrm{H}$ & 0.80004200 & -3.50503800 & 1.31419200 \\
\hline $\mathrm{H}$ & -0.97559800 & -3.46593200 & 1.20426900 \\
\hline $\mathrm{H}$ & -0.05450900 & -4.67718000 & 0.29699900 \\
\hline C & -5.08581100 & 1.27305800 & 0.19804700 \\
\hline $\mathrm{H}$ & -5.67655100 & 1.09673500 & -0.70886400 \\
\hline $\mathrm{H}$ & -5.66422200 & 0.88968300 & 1.04774200 \\
\hline $\mathrm{H}$ & -4.97459200 & 2.35044800 & 0.32651200 \\
\hline
\end{tabular}


Table S5w. Cartesian coordinates for 7A.

\begin{tabular}{|c|c|c|c|}
\hline \multirow[t]{2}{*}{ Atom } & \multicolumn{3}{|c|}{ Coordinates (Angstroms) } \\
\hline & $X$ & $\mathrm{Y}$ & $\mathrm{Z}$ \\
\hline C & -3.31689300 & -1.28016600 & -0.18479500 \\
\hline C & -2.02637200 & -1.73624600 & -0.27850800 \\
\hline C & -0.90401700 & -0.87387000 & -0.14285100 \\
\hline $\mathrm{C}$ & -1.13189400 & 0.52189800 & 0.09923300 \\
\hline C & -2.44914200 & 0.99752400 & 0.18166800 \\
\hline C & -3.52136800 & 0.12316000 & 0.04748200 \\
\hline C & 1.23762700 & 0.90217000 & 0.09283800 \\
\hline C & 1.43733800 & -0.51764500 & -0.06035000 \\
\hline C & 2.85338900 & -0.99203300 & 0.00084000 \\
\hline C & 3.51559100 & 1.39166500 & 0.01417000 \\
\hline $\mathrm{H}$ & -1.88505900 & -2.79320100 & -0.44201000 \\
\hline $\mathrm{H}$ & -2.63607100 & 2.04742700 & 0.33232400 \\
\hline $\mathrm{N}$ & 3.80507200 & 0.00215900 & -0.02041900 \\
\hline $\mathrm{N}$ & 2.20883700 & 1.78120000 & 0.10756500 \\
\hline $\mathrm{N}$ & 0.39015400 & -1.34386500 & -0.21791000 \\
\hline $\mathrm{N}$ & -0.05501800 & 1.36529900 & 0.22787900 \\
\hline 0 & 3.17847800 & -2.17539300 & 0.08189800 \\
\hline O & 4.43552400 & 2.20549100 & -0.02482300 \\
\hline C & -0.24305700 & 2.81976900 & 0.48623100 \\
\hline $\mathrm{H}$ & -1.08579900 & 2.92162600 & 1.16880000 \\
\hline $\mathrm{H}$ & 0.65318100 & 3.14725400 & 1.00795200 \\
\hline C & -0.43467900 & 3.62107500 & -0.80206800 \\
\hline $\mathrm{H}$ & 0.44694600 & 3.53772200 & -1.44188000 \\
\hline $\mathrm{H}$ & -1.31170200 & 3.29153700 & -1.36553900 \\
\hline $\mathrm{H}$ & -0.57218400 & 4.67389600 & -0.53953700 \\
\hline C & 5.20775200 & -0.44189800 & -0.00014000 \\
\hline $\mathrm{H}$ & 5.38082600 & -1.13297300 & -0.82659700 \\
\hline $\mathrm{H}$ & 5.83485000 & 0.43866600 & -0.10154000 \\
\hline $\mathrm{H}$ & 5.42117200 & -0.95377200 & 0.94105000 \\
\hline C & 0.58645000 & -2.81208000 & -0.52595900 \\
\hline $\mathrm{H}$ & 1.55590100 & -2.90550300 & -0.99846800 \\
\hline $\mathrm{H}$ & -0.17393800 & -3.06471000 & -1.26026700 \\
\hline C & 0.50942200 & -3.68029000 & 0.72766000 \\
\hline $\mathrm{H}$ & -0.44695600 & -3.58001800 & 1.24613900 \\
\hline $\mathrm{H}$ & 0.62512400 & -4.72311800 & 0.41876200 \\
\hline $\mathrm{H}$ & 1.31850400 & -3.43464900 & 1.41727200 \\
\hline O & -4.79993600 & 0.49724800 & 0.11703800 \\
\hline C & -5.14120900 & 1.87778800 & 0.35514900 \\
\hline $\mathrm{H}$ & -4.77262000 & 2.50702400 & -0.45932400 \\
\hline $\mathrm{H}$ & -6.22875600 & 1.90184900 & 0.38166300 \\
\hline $\mathrm{H}$ & -4.73530300 & 2.21001500 & 1.31449300 \\
\hline C & -4.49819500 & -2.19748700 & -0.31470600 \\
\hline
\end{tabular}




$\begin{array}{lllr}\mathrm{H} & -5.11333400 & -2.16919500 & 0.59082700 \\ \mathrm{H} & -5.14129100 & -1.88701600 & -1.14496600 \\ \mathrm{H} & -4.17380400 & -3.22517700 & -0.48721700\end{array}$

Table S5x. Cartesian coordinates for 7B.

\begin{tabular}{|c|c|c|c|}
\hline \multirow[t]{2}{*}{ Atom } & \multicolumn{3}{|c|}{ Coordinates (Angstroms) } \\
\hline & $X$ & $\mathrm{Y}$ & $\mathrm{Z}$ \\
\hline C & 3.48434800 & -0.26443400 & 0.05625300 \\
\hline $\mathrm{C}$ & 2.33656900 & -1.02612300 & 0.17051200 \\
\hline C & 1.06428000 & -0.41806400 & 0.07188300 \\
\hline $\mathrm{C}$ & 0.97399900 & 0.99252900 & -0.15992300 \\
\hline $\mathrm{C}$ & 2.16958700 & 1.74184300 & -0.25488100 \\
\hline $\mathrm{C}$ & 3.41123100 & 1.15522200 & -0.15153800 \\
\hline $\mathrm{C}$ & -1.41868800 & 0.86130900 & -0.10859300 \\
\hline C & -1.30511400 & -0.56530700 & 0.04858900 \\
\hline C & -2.59121500 & -1.33182900 & 0.02066300 \\
\hline C & -3.74560700 & 0.85854400 & 0.00784900 \\
\hline $\mathrm{H}$ & 2.42554700 & -2.08943200 & 0.31206900 \\
\hline $\mathrm{H}$ & 2.12442000 & 2.81120600 & -0.39805000 \\
\hline $\mathrm{N}$ & -3.73030700 & -0.56272700 & 0.05729600 \\
\hline $\mathrm{N}$ & -2.55578400 & 1.51543800 & -0.10748300 \\
\hline $\mathrm{N}$ & -0.10560800 & -1.15320300 & 0.18136000 \\
\hline $\mathrm{N}$ & -0.25496200 & 1.58709700 & -0.26145900 \\
\hline O & -2.65504600 & -2.55780200 & -0.04964100 \\
\hline 0 & -4.81994300 & 1.45416400 & 0.05592000 \\
\hline 0 & 4.73148500 & -0.75739000 & 0.12439000 \\
\hline C & 4.92971300 & -2.16704500 & 0.32905200 \\
\hline $\mathrm{H}$ & 4.50038900 & -2.73867900 & -0.49917800 \\
\hline $\mathrm{H}$ & 6.00903400 & -2.30543800 & 0.35422700 \\
\hline $\mathrm{H}$ & 4.49139900 & -2.48274200 & 1.28052400 \\
\hline $\mathrm{C}$ & -0.38674300 & 3.05013400 & -0.51634200 \\
\hline $\mathrm{H}$ & 0.40713600 & 3.33110000 & -1.20596400 \\
\hline $\mathrm{H}$ & -1.33927400 & 3.17599700 & -1.02594300 \\
\hline C & -0.35612700 & 3.86944800 & 0.77454600 \\
\hline $\mathrm{H}$ & -1.18815700 & 3.59459400 & 1.42687700 \\
\hline $\mathrm{H}$ & 0.58108100 & 3.73653800 & 1.32157300 \\
\hline $\mathrm{H}$ & -0.45529800 & 4.92740900 & 0.51583300 \\
\hline C & -5.00663200 & -1.29472800 & 0.06541400 \\
\hline $\mathrm{H}$ & -5.00462500 & -2.01525700 & 0.88463200 \\
\hline $\mathrm{H}$ & -5.80307200 & -0.56851600 & 0.19613900 \\
\hline $\mathrm{H}$ & -5.13332200 & -1.83093000 & -0.87810700 \\
\hline $\mathrm{C}$ & 0.02263800 & -2.62229600 & 0.50815000 \\
\hline $\mathrm{H}$ & -0.88950500 & -2.91220600 & 1.01423300 \\
\hline $\mathrm{H}$ & 0.84163500 & -2.69281800 & 1.22034800 \\
\hline C & 0.24552500 & -3.47894500 & -0.73623300 \\
\hline $\mathrm{H}$ & 1.14010600 & -3.18794200 & -1.29145000 \\
\hline
\end{tabular}




$\begin{array}{rrrr}\mathrm{H} & 0.36701300 & -4.51575000 & -0.40974400 \\ \mathrm{H} & -0.61994200 & -3.42659600 & -1.39852200 \\ \mathrm{C} & 4.67268300 & 1.95825600 & -0.24826400 \\ \mathrm{H} & 5.27077400 & 1.84482400 & 0.66219700 \\ \mathrm{H} & 5.29277500 & 1.59946400 & -1.07687300 \\ \mathrm{H} & 4.45385400 & 3.01609300 & -0.40099200\end{array}$

Table S5y. Cartesian coordinates for 8A.

\begin{tabular}{|c|c|c|c|}
\hline \multirow[t]{2}{*}{ Atom } & \multicolumn{3}{|c|}{ Coordinates (Angstroms) } \\
\hline & $\mathrm{X}$ & $\mathrm{Y}$ & $\mathrm{Z}$ \\
\hline $\mathrm{C}$ & -1.48809700 & 1.73260000 & 0.05905100 \\
\hline $\mathrm{C}$ & -0.37541300 & 0.86170600 & -0.00448200 \\
\hline $\mathrm{C}$ & -0.59571800 & -0.52043700 & -0.29419500 \\
\hline C & -1.91604300 & -0.97203100 & -0.43992200 \\
\hline C & 1.75892800 & -0.93557900 & -0.14736500 \\
\hline C & 1.96193900 & 0.48388400 & 0.05061300 \\
\hline $\mathrm{C}$ & 3.38966400 & 0.94312500 & 0.09215800 \\
\hline $\mathrm{C}$ & 4.02341300 & -1.44722900 & 0.07919700 \\
\hline $\mathrm{H}$ & -1.33848300 & 2.79172300 & 0.19972500 \\
\hline $\mathrm{H}$ & -2.12106400 & -2.02103200 & -0.59440700 \\
\hline $\mathrm{N}$ & 4.32495500 & -0.06088000 & 0.16382400 \\
\hline $\mathrm{N}$ & 2.72170700 & -1.82080400 & -0.11233600 \\
\hline $\mathrm{N}$ & 0.92471400 & 1.31834800 & 0.16525000 \\
\hline $\mathrm{N}$ & 0.47615500 & -1.37791300 & -0.37751500 \\
\hline O & 3.72391500 & 2.12422900 & 0.04788800 \\
\hline 0 & 4.92942100 & -2.27089000 & 0.16299100 \\
\hline $\mathrm{N}$ & -4.27921200 & -0.63619800 & -0.52241500 \\
\hline $\mathrm{H}$ & -4.35338300 & -1.38061000 & -1.20556800 \\
\hline $\mathrm{C}$ & -2.99291900 & -0.10214100 & -0.33407800 \\
\hline C & -2.78705900 & 1.29815800 & -0.11873400 \\
\hline C & -5.34182600 & -0.50826900 & 0.36104100 \\
\hline C & -6.62087200 & -1.18451400 & -0.07447800 \\
\hline $\mathrm{H}$ & -7.11184200 & -1.61189200 & 0.80148100 \\
\hline $\mathrm{H}$ & -7.28557000 & -0.42440900 & -0.49932300 \\
\hline $\mathrm{H}$ & -6.46482700 & -1.96130300 & -0.82619600 \\
\hline 0 & -5.24434200 & 0.12690000 & 1.40630900 \\
\hline C & 5.73134700 & 0.36558000 & 0.24448900 \\
\hline $\mathrm{H}$ & 6.33753900 & -0.52210500 & 0.39738200 \\
\hline $\mathrm{H}$ & 6.02029500 & 0.86750200 & -0.68189700 \\
\hline $\mathrm{H}$ & 5.85041700 & 1.06010300 & 1.07725300 \\
\hline C & 0.28612800 & -2.82284700 & -0.68296400 \\
\hline $\mathrm{H}$ & -0.52100500 & -2.89519400 & -1.41080300 \\
\hline $\mathrm{H}$ & 1.20568500 & -3.14771700 & -1.16489800 \\
\hline C & 0.01584700 & -3.65486100 & 0.57156700 \\
\hline $\mathrm{H}$ & 0.86034500 & -3.59873300 & 1.26231200 \\
\hline $\mathrm{H}$ & -0.88847600 & -3.32988200 & 1.09286900 \\
\hline
\end{tabular}




$\begin{array}{rrrr}\mathrm{H} & -0.11719100 & -4.69862300 & 0.27315200 \\ \mathrm{C} & 1.11121900 & 2.77430700 & 0.53535900 \\ \mathrm{H} & 2.04372800 & 2.84100800 & 1.08157300 \\ \mathrm{H} & 0.29926100 & 3.00695100 & 1.21987000 \\ \mathrm{C} & 1.13354600 & 3.68856300 & -0.68766200 \\ \mathrm{H} & 1.23172300 & 4.71727100 & -0.32926900 \\ \mathrm{H} & 1.99103400 & 3.46290100 & -1.32293300 \\ \mathrm{H} & 0.21865700 & 3.61795500 & -1.27985900 \\ \mathrm{C} & -3.91047600 & 2.29659800 & -0.16264100 \\ \mathrm{H} & -4.60752100 & 2.05872200 & -0.97204300 \\ \mathrm{H} & -4.48110900 & 2.28473500 & 0.76989500 \\ \mathrm{H} & -3.52003400 & 3.30315500 & -0.32519400\end{array}$

Table S5z. Cartesian coordinates for 8B.

\begin{tabular}{|c|c|c|c|}
\hline \multirow[t]{2}{*}{ Atom } & \multicolumn{3}{|c|}{ Coordinates (Angstroms) } \\
\hline & $X$ & $\mathrm{Y}$ & $\mathrm{Z}$ \\
\hline $\mathrm{C}$ & 2.00106100 & -0.52088200 & 0.09342600 \\
\hline $\mathrm{C}$ & 0.65553100 & -0.10950700 & 0.01409600 \\
\hline $\mathrm{C}$ & 0.34728800 & 1.27002500 & -0.19422700 \\
\hline C & 1.41617900 & 2.18483600 & -0.30045000 \\
\hline C & -1.99400900 & 0.77929500 & -0.09468500 \\
\hline C & -1.66027900 & -0.62062600 & 0.02201300 \\
\hline C & -2.81777800 & -1.57380500 & -0.01046900 \\
\hline C & -4.28885700 & 0.41309000 & 0.08878000 \\
\hline $\mathrm{H}$ & 2.27241700 & -1.55253500 & 0.22933400 \\
\hline $\mathrm{H}$ & 1.21176400 & 3.23655900 & -0.43435400 \\
\hline $\mathrm{N}$ & -4.05737900 & -0.98997400 & 0.09293500 \\
\hline $\mathrm{N}$ & -3.21652900 & 1.24818500 & -0.04479700 \\
\hline $\mathrm{N}$ & -0.38767800 & -1.02214400 & 0.12105800 \\
\hline $\mathrm{N}$ & -0.96030000 & 1.67539100 & -0.26259500 \\
\hline O & -2.69431100 & -2.78983100 & -0.13632100 \\
\hline O & -5.43740700 & 0.83701600 & 0.18887800 \\
\hline C & 2.73601200 & 1.79073800 & -0.21817400 \\
\hline C & 3.03209200 & 0.39762400 & -0.02192100 \\
\hline C & -5.20793200 & -1.90738800 & 0.11028500 \\
\hline $\mathrm{H}$ & -6.09661400 & -1.31917600 & 0.31797300 \\
\hline $\mathrm{H}$ & -5.30116800 & -2.40560500 & -0.85774300 \\
\hline $\mathrm{H}$ & -5.05529100 & -2.66109400 & 0.88390000 \\
\hline C & -1.31673000 & 3.10541500 & -0.48386400 \\
\hline $\mathrm{H}$ & -0.59225300 & 3.51495400 & -1.18583100 \\
\hline $\mathrm{H}$ & -2.29027800 & 3.09620200 & -0.96881500 \\
\hline C & -1.37561300 & 3.89699200 & 0.82304200 \\
\hline $\mathrm{H}$ & -2.13951900 & 3.48818500 & 1.48843100 \\
\hline $\mathrm{H}$ & -0.41498800 & 3.89679000 & 1.34501400 \\
\hline $\mathrm{H}$ & -1.63900900 & 4.93246300 & 0.58958200 \\
\hline
\end{tabular}




$\begin{array}{rrrr}C & -0.02736300 & -2.46387400 & 0.39784200 \\ H & -0.87695600 & -2.90972400 & 0.89910300 \\ H & 0.80314500 & -2.42850700 & 1.09891800 \\ C & 0.31368900 & -3.23084300 & -0.87812400 \\ H & 0.59116900 & -4.24871000 & -0.58963700 \\ H & -0.55312700 & -3.28483400 & -1.53853900 \\ H & 1.15295200 & -2.78770300 & -1.41838800 \\ C & 3.83137800 & 2.81490200 & -0.32805000 \\ H & 4.45120200 & 2.83550100 & 0.57639200 \\ H & 4.48837200 & 2.60806900 & -1.18158300 \\ H & 3.41409800 & 3.81282400 & -0.46718900 \\ \text { N } & 4.37525800 & 0.02600000 & 0.04721000 \\ H & 5.04565700 & 0.77219700 & -0.07214200 \\ C & 4.92197300 & -1.22934800 & 0.25584300 \\ O & 4.23509300 & -2.23571000 & 0.42147600 \\ C & 6.43289500 & -1.27869000 & 0.24362100 \\ H & 6.90595300 & -0.29746900 & 0.31874100 \\ H & 6.76737900 & -1.90657500 & 1.07223400 \\ H & 6.75725500 & -1.75353200 & -0.68824100 \\ -------------------------------------------------------------------------------------------------------------\end{array}$

Table S5aa. Cartesian coordinates for 9A.

\begin{tabular}{|c|c|c|c|}
\hline \multirow[t]{2}{*}{ Atom } & \multicolumn{3}{|c|}{ Coordinates (Angstroms) } \\
\hline & $X$ & $\mathrm{Y}$ & $\mathrm{Z}$ \\
\hline C & 3.28270000 & 1.33432600 & -0.19788600 \\
\hline C & 1.98084100 & 1.77959600 & -0.28939400 \\
\hline C & 0.87713800 & 0.90500800 & -0.14430000 \\
\hline $\mathrm{C}$ & 1.12522800 & -0.48000800 & 0.10293100 \\
\hline C & 2.45975800 & -0.92451000 & 0.18367300 \\
\hline C & 3.53152600 & -0.06024400 & 0.04072300 \\
\hline $\mathrm{C}$ & -1.23246600 & -0.91002800 & 0.09184800 \\
\hline C & -1.45681800 & 0.51089200 & -0.05226400 \\
\hline $\mathrm{C}$ & -2.88588200 & 0.95927800 & 0.02362400 \\
\hline C & -3.50210200 & -1.43576200 & -0.01174300 \\
\hline $\mathrm{H}$ & 1.82235800 & 2.83314800 & -0.46022100 \\
\hline $\mathrm{H}$ & 2.65614800 & -1.97226200 & 0.34515900 \\
\hline $\mathrm{N}$ & -3.81735900 & -0.04942800 & -0.02872500 \\
\hline $\mathrm{N}$ & -2.18977900 & -1.80363400 & 0.09055600 \\
\hline $\mathrm{N}$ & -0.43360300 & 1.35594700 & -0.21284100 \\
\hline $\mathrm{N}$ & 0.06586600 & -1.34586000 & 0.23460300 \\
\hline 0 & -3.22407600 & 2.13463200 & 0.14051900 \\
\hline 0 & -4.40734800 & -2.26309100 & -0.07196600 \\
\hline C & 0.28014100 & -2.79658300 & 0.49761900 \\
\hline $\mathrm{H}$ & 1.12375300 & -2.88060200 & 1.18085800 \\
\hline $\mathrm{H}$ & -0.61179700 & -3.13816600 & 1.01809100 \\
\hline $\mathrm{C}$ & 0.48956800 & -3.59728700 & -0.78814900 \\
\hline $\mathrm{H}$ & -0.38868200 & -3.52765300 & -1.43418000 \\
\hline
\end{tabular}




$\begin{array}{rrrr}\mathrm{H} & 1.36619100 & -3.25628300 & -1.34529100 \\ \mathrm{H} & 0.63980200 & -4.64743200 & -0.52199100 \\ \mathrm{C} & -5.22867500 & 0.36738900 & -0.00647300 \\ \mathrm{H} & -5.83731300 & -0.51629600 & -0.17250600 \\ \mathrm{H} & -5.46864500 & 0.81635700 & 0.96032400 \\ \mathrm{H} & -5.39843300 & 1.10447200 & -0.79247200 \\ \mathrm{C} & -0.65422100 & 2.82159800 & -0.51443400 \\ \mathrm{H} & 0.09428100 & 3.08348600 & -1.25788800 \\ \mathrm{H} & -1.63071300 & 2.90460700 & -0.97428800 \\ \mathrm{C} & -0.57016800 & 3.68892000 & 0.73970000 \\ \mathrm{H} & 0.39389200 & 3.59872100 & 1.24526500 \\ \mathrm{H} & -0.70119700 & 4.73050400 & 0.43282300 \\ \mathrm{H} & -1.36769600 & 3.43363300 & 1.43913600 \\ \mathrm{C} & 5.16209100 & -2.04931500 & 0.37408700 \\ \mathrm{H} & 6.23301000 & -2.26653300 & 0.41439600 \\ \mathrm{H} & 4.72459000 & -2.36902300 & 1.32560800 \\ \mathrm{H} & 4.73176100 & -2.65973500 & -0.42669600 \\ \mathrm{C} & 4.41879500 & 2.31028100 & -0.35015100 \\ \mathrm{H} & 5.05393200 & 2.32251900 & 0.54235600 \\ \mathrm{H} & 5.06117100 & 2.04080100 & -1.19588300 \\ \mathrm{H} & 4.04537700 & 3.32238400 & -0.51621400 \\ \mathrm{C} & 4.95659300 & -0.55400400 & 0.12540200 \\ \mathrm{H} & 5.46223700 & 0.01933300 & 0.91422200 \\ \mathrm{H} & 5.46721400 & -0.26476600 & -0.80305700 \\ ---------------------------------------------------------\end{array}$

Table S5ab. Cartesian coordinates for 9B.

\begin{tabular}{|c|c|c|c|}
\hline \multirow[t]{2}{*}{ Atom } & \multicolumn{3}{|c|}{ Coordinates (Angstroms) } \\
\hline & $X$ & $\mathrm{Y}$ & $\mathrm{Z}$ \\
\hline C & -3.45356300 & -0.36585700 & -0.32010800 \\
\hline C & -2.27060900 & -1.07189800 & -0.40953200 \\
\hline $\mathrm{C}$ & -1.01118500 & -0.45664100 & -0.21733700 \\
\hline $\mathrm{C}$ & -0.96516200 & 0.94004500 & 0.08363900 \\
\hline $\mathrm{C}$ & -2.17672800 & 1.65430800 & 0.14992500 \\
\hline C & -3.40314700 & 1.04084300 & -0.04759200 \\
\hline C & 1.42913700 & 0.85850800 & 0.13733700 \\
\hline C & 1.35107700 & -0.56954600 & -0.07920700 \\
\hline C & 2.65212900 & -1.31455700 & -0.01871700 \\
\hline C & 3.76092500 & 0.89302500 & 0.09918400 \\
\hline $\mathrm{H}$ & -2.33475500 & -2.12959300 & -0.61660200 \\
\hline $\mathrm{H}$ & -2.16223300 & 2.71722200 & 0.34083700 \\
\hline $\mathrm{N}$ & 3.77641100 & -0.52507600 & -0.00140600 \\
\hline $\mathrm{N}$ & 2.55382600 & 1.52596500 & 0.20098400 \\
\hline $\mathrm{N}$ & 0.17749000 & -1.16991100 & -0.29673400 \\
\hline $\mathrm{N}$ & 0.24954700 & 1.55473400 & 0.27269500 \\
\hline 0 & 2.73163800 & -2.53942400 & 0.02978200 \\
\hline
\end{tabular}




$\begin{array}{lrrr}\mathrm{O} & 4.82197600 & 1.51063600 & 0.10711900 \\ \mathrm{C} & 0.33816100 & 3.00724500 & 0.59159400 \\ \mathrm{H} & -0.49239600 & 3.24332900 & 1.25483600 \\ \mathrm{H} & 1.26365700 & 3.13303500 & 1.14912700 \\ \mathrm{C} & 0.34545700 & 3.87857600 & -0.66526900 \\ \mathrm{H} & 1.21113900 & 3.64786300 & -1.29036200 \\ \mathrm{H} & -0.56376300 & 3.74708400 & -1.25777800 \\ \mathrm{H} & 0.40859600 & 4.92712400 & -0.36078800 \\ \mathrm{C} & 5.06721300 & -1.23212600 & 0.01169800 \\ \mathrm{H} & 5.85183000 & -0.49026100 & -0.10146900 \\ \mathrm{H} & 5.18763700 & -1.76927300 & 0.95560900 \\ \mathrm{H} & 5.09290200 & -1.94852400 & -0.81038000 \\ \mathrm{C} & 0.09163800 & -2.62944900 & -0.68446800 \\ \mathrm{H} & -0.68694200 & -2.68444800 & -1.44104100 \\ \mathrm{H} & 1.03480100 & -2.88594900 & -1.15015200 \\ \mathrm{C} & -0.18633700 & -3.53430700 & 0.51353300 \\ \mathrm{H} & -1.11735500 & -3.27747300 & 1.02373700 \\ \mathrm{H} & -0.27075200 & -4.55949300 & 0.14168100 \\ \mathrm{H} & 0.63744600 & -3.49484200 & 1.22760100 \\ \mathrm{C} & -4.76655600 & -1.09869300 & -0.48244400 \\ \mathrm{H} & -5.49479200 & -0.45304800 & -0.98204600 \\ \mathrm{H} & -4.61059400 & -1.96133000 & -1.13800400 \\ \mathrm{C} & -5.34836000 & -1.58889000 & 0.86013100 \\ \mathrm{H} & -4.66509100 & -2.29223300 & 1.34629300 \\ \mathrm{H} & -5.52796300 & -0.76042400 & 1.55214000 \\ \mathrm{H} & -6.30092100 & -2.09976700 & 0.68980800 \\ \mathrm{C} & -4.65778700 & 1.86924200 & -0.00091300 \\ \mathrm{H} & -5.15561000 & 1.86947700 & -0.97752800 \\ \mathrm{H} & -5.37490900 & 1.46946000 & 0.72269200 \\ \mathrm{H} & -4.43758000 & 2.90341700 & 0.26905600 \\ -------------------------------------------------------------------------\end{array}$

Table S6. Cartesian coordinates for all $\mathrm{S}_{1}$ excited state $\mathrm{Et}-\mathrm{Fl}^{+}$derivatives optimized at B3LYP/6$31+\mathrm{G}(\mathrm{d}, \mathrm{p})$ with implicit solvation of acetonitrile.

Table S6a. Cartesian coordinates for Et- $\mathrm{Fl}^{+}$.

\begin{tabular}{|c|c|c|c|}
\hline \multirow[t]{2}{*}{ Atom } & \multicolumn{3}{|c|}{ Coordinates (Angstroms) } \\
\hline & $X$ & $\mathrm{Y}$ & $\mathrm{Z}$ \\
\hline $\mathrm{C}$ & -3.66843000 & -0.80078800 & -0.01934000 \\
\hline
\end{tabular}




\begin{tabular}{|c|c|c|c|}
\hline C & -2.39251600 & -1.39597100 & -0.15971400 \\
\hline C & -1.20510500 & -0.66540100 & -0.10215000 \\
\hline $\mathrm{C}$ & -1.29313300 & 0.73637100 & 0.12229300 \\
\hline $\mathrm{C}$ & -2.57665200 & 1.32902400 & 0.24987800 \\
\hline C & -3.75797100 & 0.59568700 & 0.18547500 \\
\hline C & 1.08396000 & 0.87838400 & 0.11361400 \\
\hline C & 1.17966300 & -0.53655600 & -0.06878500 \\
\hline C & 2.51718300 & -1.12856000 & -0.00474000 \\
\hline C & 3.42686900 & 1.15180400 & 0.12116000 \\
\hline $\mathrm{H}$ & -2.36331100 & -2.46507200 & -0.31077700 \\
\hline $\mathrm{H}$ & -2.65356500 & 2.39637000 & 0.40077800 \\
\hline $\mathrm{N}$ & 3.58531900 & -0.22807900 & 0.06605000 \\
\hline $\mathrm{N}$ & 2.14181500 & 1.66692100 & 0.20980500 \\
\hline $\mathrm{N}$ & -0.14361100 & 1.49843200 & 0.20181700 \\
\hline O & 2.74746400 & -2.34086700 & -0.00722600 \\
\hline 0 & 4.38748700 & 1.92176100 & 0.10994800 \\
\hline C & -4.89240700 & -1.65768000 & -0.09511300 \\
\hline $\mathrm{H}$ & -5.48140000 & -1.57520100 & 0.82719400 \\
\hline $\mathrm{H}$ & -5.54992100 & -1.32561700 & -0.90867800 \\
\hline $\mathrm{H}$ & -4.64430700 & -2.70760800 & -0.25642300 \\
\hline $\mathrm{C}$ & -5.08927800 & 1.28099500 & 0.33058300 \\
\hline $\mathrm{H}$ & -5.70307200 & 1.14135300 & -0.56642200 \\
\hline $\mathrm{H}$ & -5.65436500 & 0.86765500 & 1.17328000 \\
\hline $\mathrm{H}$ & -4.96566300 & 2.35279300 & 0.49594700 \\
\hline C & -0.20430900 & 2.97952500 & 0.35554100 \\
\hline $\mathrm{H}$ & -0.98752300 & 3.19934200 & 1.08001300 \\
\hline $\mathrm{H}$ & 0.74315800 & 3.28009700 & 0.79222100 \\
\hline C & -0.42933900 & 3.69432900 & -0.97892300 \\
\hline $\mathrm{H}$ & 0.39780000 & 3.49821000 & -1.66602400 \\
\hline $\mathrm{H}$ & -1.36386200 & 3.39209500 & -1.45862300 \\
\hline $\mathrm{H}$ & -0.47335400 & 4.77101500 & -0.79103500 \\
\hline C & 0.08667300 & -2.70626100 & -0.71198800 \\
\hline $\mathrm{H}$ & -0.72404500 & -2.82529700 & -1.43122100 \\
\hline $\mathrm{H}$ & 1.02214100 & -2.83920700 & -1.24547600 \\
\hline C & -0.01470200 & -3.71499600 & 0.43425600 \\
\hline $\mathrm{H}$ & 0.83773200 & -3.61449200 & 1.10805500 \\
\hline $\mathrm{H}$ & -0.93708700 & -3.60191500 & 1.00974600 \\
\hline $\mathrm{H}$ & -0.00064500 & -4.72210300 & 0.00673900 \\
\hline $\mathrm{N}$ & 0.04138800 & -1.28604700 & -0.25893300 \\
\hline $\mathrm{C}$ & 4.93788700 & -0.80566400 & 0.08274600 \\
\hline $\mathrm{H}$ & 5.11579300 & -1.35769900 & -0.84231700 \\
\hline $\mathrm{H}$ & 5.64722700 & 0.01204800 & 0.17258200 \\
\hline $\mathrm{H}$ & 5.03392200 & -1.48795200 & 0.92872300 \\
\hline
\end{tabular}

Table S6b. Cartesian coordinates for 1AB.

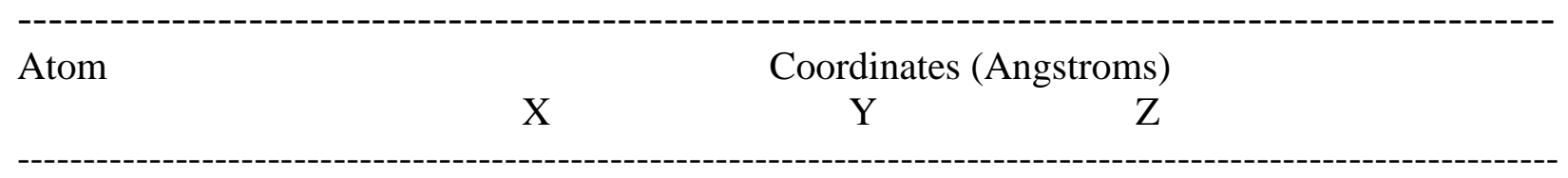




\begin{tabular}{|c|c|c|c|}
\hline C & -2.46514200 & -0.70627900 & 0.07317500 \\
\hline C & -1.22558500 & -1.33423000 & 0.17607800 \\
\hline $\mathrm{C}$ & -0.02101100 & -0.62412400 & 0.08932500 \\
\hline C & -0.08633400 & 0.78343100 & -0.11456500 \\
\hline $\mathrm{C}$ & -1.34024300 & 1.40728300 & -0.21451000 \\
\hline C & -2.52725800 & 0.68879000 & -0.13408900 \\
\hline C & 2.28082300 & 0.87823100 & -0.14097900 \\
\hline C & 2.36137600 & -0.54029600 & 0.00912700 \\
\hline C & 3.68564300 & -1.15842100 & -0.10080500 \\
\hline C & 4.62582800 & 1.10085700 & -0.05439500 \\
\hline $\mathrm{H}$ & -1.21250400 & -2.40176100 & 0.32255500 \\
\hline $\mathrm{H}$ & -1.40130300 & 2.47408400 & -0.36002600 \\
\hline $\mathrm{N}$ & 4.77426600 & -0.26786600 & -0.13344100 \\
\hline $\mathrm{N}$ & 3.37568100 & 1.63433800 & -0.30283800 \\
\hline $\mathrm{N}$ & 1.20626500 & -1.26694700 & 0.21656400 \\
\hline $\mathrm{N}$ & 1.09055600 & 1.52445400 & -0.19262300 \\
\hline O & 3.88959600 & -2.36847200 & -0.15583800 \\
\hline O & 5.55566800 & 1.86962200 & 0.19313400 \\
\hline C & -3.82009100 & 1.48347300 & -0.23540000 \\
\hline C & -3.69273300 & -1.60553200 & 0.16200300 \\
\hline $\mathrm{F}$ & -3.60376800 & 2.70495700 & -0.77388200 \\
\hline $\mathrm{F}$ & -4.37846500 & 1.68806700 & 0.98174300 \\
\hline $\mathrm{F}$ & -4.66481500 & -1.06822900 & 0.92761800 \\
\hline $\mathrm{F}$ & -3.38620300 & -2.80649600 & 0.70016000 \\
\hline $\mathrm{F}$ & -4.21692300 & -1.84664300 & -1.06200400 \\
\hline $\mathrm{F}$ & -4.74202600 & 0.87127700 & -1.00849900 \\
\hline C & 1.06043200 & 3.01597300 & -0.26401700 \\
\hline $\mathrm{H}$ & 2.01338800 & 3.31463000 & -0.69374300 \\
\hline $\mathrm{H}$ & 0.27394800 & 3.28733900 & -0.96640600 \\
\hline C & 0.86680100 & 3.65749900 & 1.11248300 \\
\hline $\mathrm{H}$ & -0.06402600 & 3.34035200 & 1.58933000 \\
\hline $\mathrm{H}$ & 1.70371200 & 3.41647700 & 1.77253600 \\
\hline $\mathrm{H}$ & 0.83226700 & 4.74201900 & 0.97869800 \\
\hline C & 6.13187400 & -0.83368700 & -0.14602300 \\
\hline $\mathrm{H}$ & 6.14510300 & -1.69522900 & -0.81045500 \\
\hline $\mathrm{H}$ & 6.81526800 & -0.06306300 & -0.49904000 \\
\hline $\mathrm{H}$ & 6.42204600 & -1.14111800 & 0.86217300 \\
\hline C & 1.23675500 & -2.69577900 & 0.65616700 \\
\hline $\mathrm{H}$ & 2.18347200 & -2.84757000 & 1.16415500 \\
\hline $\mathrm{H}$ & 0.44518200 & -2.80265700 & 1.39838200 \\
\hline C & 1.08343900 & -3.68930500 & -0.49702100 \\
\hline $\mathrm{H}$ & 1.08933600 & -4.69893800 & -0.07616900 \\
\hline $\mathrm{H}$ & 0.14828700 & -3.55513800 & -1.04652200 \\
\hline $\mathrm{H}$ & 1.91871900 & -3.60019300 & -1.19301000 \\
\hline
\end{tabular}

Table S6c. Cartesian coordinates for 2AB.

Atom

\section{X}

Coordinates (Angstroms)
Y 


\begin{tabular}{|c|c|c|c|}
\hline C & -1.66793400 & 1.33563300 & 0.17734400 \\
\hline $\mathrm{C}$ & -0.45974900 & 0.62443100 & 0.08728700 \\
\hline C & -0.51723100 & -0.79003500 & -0.13112100 \\
\hline C & -1.75961800 & -1.42840200 & -0.22666600 \\
\hline C & 1.85135400 & -0.86407300 & -0.15383100 \\
\hline C & 1.91958000 & 0.55543700 & 0.01780500 \\
\hline C & 3.24374900 & 1.18519300 & -0.06880800 \\
\hline C & 4.19658800 & -1.06869300 & -0.04721500 \\
\hline $\mathrm{H}$ & -1.67532000 & 2.40549700 & 0.31847000 \\
\hline $\mathrm{H}$ & -1.84486300 & -2.49501500 & -0.36852700 \\
\hline $\mathrm{N}$ & 4.33611800 & 0.30304300 & -0.10369500 \\
\hline $\mathrm{N}$ & 2.95117900 & -1.60762500 & -0.31569600 \\
\hline $\mathrm{N}$ & 0.75842100 & 1.26942200 & 0.21844300 \\
\hline $\mathrm{N}$ & 0.66601500 & -1.51690700 & -0.22218900 \\
\hline O & 3.43770300 & 2.39643700 & -0.10553100 \\
\hline O & 5.12788200 & -1.83424200 & 0.19730600 \\
\hline C & -2.88213200 & 0.68079300 & 0.06556300 \\
\hline C & -2.93978400 & -0.70536200 & -0.13304200 \\
\hline $\mathrm{N}$ & -4.08717800 & 1.53781400 & 0.15284900 \\
\hline O & -4.48507800 & 1.81164800 & 1.27693700 \\
\hline 0 & -4.54092000 & 1.95320500 & -0.90475100 \\
\hline C & 0.64676900 & -3.00728800 & -0.33395700 \\
\hline $\mathrm{H}$ & -0.14363200 & -3.26306300 & -1.03781300 \\
\hline $\mathrm{H}$ & 1.59840300 & -3.28549000 & -0.77999500 \\
\hline C & 0.46975600 & -3.68961300 & 1.02496400 \\
\hline $\mathrm{H}$ & 1.30970500 & -3.46142100 & 1.68547400 \\
\hline $\mathrm{H}$ & -0.46002900 & -3.39719200 & 1.51897600 \\
\hline $\mathrm{H}$ & 0.44420600 & -4.76966100 & 0.85766000 \\
\hline C & 0.77569700 & 2.70572500 & 0.64946500 \\
\hline $\mathrm{H}$ & -0.02129700 & 2.80993500 & 1.38559600 \\
\hline $\mathrm{H}$ & 1.71729300 & 2.86539100 & 1.16372000 \\
\hline C & 0.62576400 & 3.68658400 & -0.51409500 \\
\hline $\mathrm{H}$ & 1.46902600 & 3.59950400 & -1.20056900 \\
\hline $\mathrm{H}$ & -0.30234200 & 3.54118100 & -1.07267100 \\
\hline $\mathrm{H}$ & 0.61926900 & 4.69903300 & -0.10040000 \\
\hline $\mathrm{C}$ & 5.69017700 & 0.87898000 & -0.09737400 \\
\hline $\mathrm{H}$ & 6.38327200 & 0.11345100 & -0.44198000 \\
\hline $\mathrm{H}$ & 5.70614100 & 1.74130100 & -0.76084700 \\
\hline $\mathrm{H}$ & 5.96346800 & 1.18708700 & 0.91518200 \\
\hline $\mathrm{N}$ & -4.20391400 & -1.43058300 & -0.22829300 \\
\hline O & -5.24166100 & -0.78746600 & -0.05389300 \\
\hline O & -4.15614000 & -2.63615200 & -0.47133200 \\
\hline
\end{tabular}

Table S6d. Cartesian coordinates for 3AB.

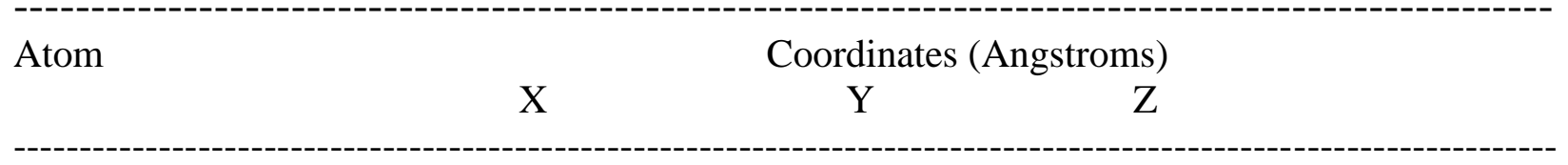




\begin{tabular}{|c|c|c|c|}
\hline C & 3.33310400 & -0.77112300 & 0.04775200 \\
\hline C & 2.07233500 & -1.38233200 & 0.16915900 \\
\hline C & 0.88716100 & -0.65598900 & 0.09635800 \\
\hline C & 0.97126900 & 0.75983100 & -0.11590600 \\
\hline C & 2.23458600 & 1.36361600 & -0.22583600 \\
\hline C & 3.41717500 & 0.62342300 & -0.15224000 \\
\hline C & -1.40089000 & 0.88459200 & -0.11678500 \\
\hline C & -1.49651600 & -0.53642500 & 0.03805800 \\
\hline C & -2.83044300 & -1.13694900 & -0.06938600 \\
\hline C & -3.74526300 & 1.13737100 & -0.05205200 \\
\hline $\mathrm{H}$ & 2.06993500 & -2.45236300 & 0.31542200 \\
\hline $\mathrm{H}$ & 2.33487400 & 2.43005900 & -0.36966500 \\
\hline $\mathrm{N}$ & -3.90319900 & -0.23450700 & -0.12632300 \\
\hline $\mathrm{N}$ & -2.47101600 & 1.66220100 & -0.23225500 \\
\hline $\mathrm{N}$ & -0.35495700 & -1.27748600 & 0.23350800 \\
\hline $\mathrm{N}$ & -0.18895700 & 1.51287700 & -0.18980500 \\
\hline O & -3.04660500 & -2.34670400 & -0.11070600 \\
\hline O & -4.68534600 & 1.90631200 & 0.13550600 \\
\hline C & 4.52446200 & -1.67173100 & 0.13826000 \\
\hline $\mathrm{H}$ & 5.51975100 & -1.21712100 & 0.04005800 \\
\hline C & 4.70038600 & 1.37458900 & -0.28495600 \\
\hline $\mathrm{H}$ & 5.63665300 & 0.80517000 & -0.20173000 \\
\hline O & 4.41054000 & -2.87377700 & 0.31186000 \\
\hline 0 & 4.72948000 & 2.57963900 & -0.47637500 \\
\hline C & -0.13568700 & 3.00177600 & -0.29722500 \\
\hline $\mathrm{H}$ & 0.65050000 & 3.24223100 & -1.01104900 \\
\hline $\mathrm{H}$ & -1.08483500 & 3.30808200 & -0.72738500 \\
\hline C & 0.08125100 & 3.67359000 & 1.06113800 \\
\hline $\mathrm{H}$ & -0.75161800 & 3.46029000 & 1.73558300 \\
\hline $\mathrm{H}$ & 1.01267400 & 3.35608200 & 1.53641400 \\
\hline $\mathrm{H}$ & 0.12871100 & 4.75419700 & 0.90102300 \\
\hline C & -5.26678000 & -0.78567500 & -0.16025500 \\
\hline $\mathrm{H}$ & -5.59527600 & -1.04576100 & 0.84963600 \\
\hline $\mathrm{H}$ & -5.92858100 & -0.02603600 & -0.57165400 \\
\hline $\mathrm{H}$ & -5.26678600 & -1.67643900 & -0.78485000 \\
\hline C & -0.39821300 & -2.71020700 & 0.65960800 \\
\hline $\mathrm{H}$ & -1.34234700 & -2.85648200 & 1.17374100 \\
\hline $\mathrm{H}$ & 0.39982500 & -2.83213300 & 1.39216700 \\
\hline $\mathrm{C}$ & -0.26536200 & -3.69406400 & -0.50420200 \\
\hline $\mathrm{H}$ & -0.27697600 & -4.70773700 & -0.09319300 \\
\hline $\mathrm{H}$ & -1.10606500 & -3.59052700 & -1.19173300 \\
\hline $\mathrm{H}$ & 0.66647500 & -3.56262500 & -1.05987100 \\
\hline
\end{tabular}

Table S6e. Cartesian coordinates for $4 \mathrm{AB}$.

\begin{tabular}{|c|c|c|c|}
\hline \multirow[t]{2}{*}{ Atom } & \multicolumn{3}{|c|}{ Coordinates (Angstroms) } \\
\hline & $\mathrm{X}$ & $\mathrm{Y}$ & $\mathrm{Z}$ \\
\hline $\mathrm{C}$ & 2.41952200 & -1.41577900 & -0.17788200 \\
\hline
\end{tabular}




\begin{tabular}{|c|c|c|c|}
\hline C & 1.23798800 & -0.67166300 & -0.09900400 \\
\hline C & 1.32716800 & 0.73761600 & 0.14425900 \\
\hline $\mathrm{C}$ & 2.59342100 & 1.34550700 & 0.28250200 \\
\hline $\mathrm{C}$ & -1.04394600 & 0.87222000 & 0.13717900 \\
\hline C & -1.14667600 & -0.53672500 & -0.05595700 \\
\hline C & -2.48198500 & -1.13540800 & 0.02738500 \\
\hline C & -3.38847800 & 1.14155500 & 0.09513900 \\
\hline $\mathrm{H}$ & 2.42352900 & -2.48222600 & -0.34611700 \\
\hline $\mathrm{H}$ & 2.70554200 & 2.40631800 & 0.45243800 \\
\hline $\mathrm{N}$ & -3.55205500 & -0.23183900 & 0.11235500 \\
\hline N & -2.11418400 & 1.65691400 & 0.27750200 \\
\hline $\mathrm{N}$ & -0.00530000 & -1.28345200 & -0.26496700 \\
\hline $\mathrm{N}$ & 0.16831100 & 1.49430300 & 0.22542200 \\
\hline 0 & -2.70250000 & -2.34624100 & 0.02570300 \\
\hline 0 & -4.33271700 & 1.91813700 & -0.04853100 \\
\hline $\mathrm{C}$ & 3.65264600 & -0.79089800 & -0.02795200 \\
\hline C & 3.74021000 & 0.58399700 & 0.20010400 \\
\hline $\mathrm{F}$ & 4.76902300 & -1.51353900 & -0.10497700 \\
\hline $\mathrm{F}$ & 4.94431700 & 1.15276900 & 0.33273500 \\
\hline C & 0.22965800 & 2.98008000 & 0.36156500 \\
\hline $\mathrm{H}$ & -0.72124800 & 3.28510100 & 0.78808100 \\
\hline $\mathrm{H}$ & 1.00818000 & 3.20321400 & 1.08985300 \\
\hline $\mathrm{C}$ & 0.46480300 & 3.67691300 & -0.98086400 \\
\hline $\mathrm{H}$ & 1.39810000 & 3.36324300 & -1.45502100 \\
\hline $\mathrm{H}$ & -0.36281400 & 3.48194200 & -1.66738600 \\
\hline $\mathrm{H}$ & 0.51737400 & 4.75415400 & -0.80028000 \\
\hline C & -4.91907700 & -0.77461700 & 0.12287000 \\
\hline $\mathrm{H}$ & -4.91351600 & -1.71726400 & 0.66547700 \\
\hline $\mathrm{H}$ & -5.56841900 & -0.05154800 & 0.61296600 \\
\hline $\mathrm{H}$ & -5.26908200 & -0.94149200 & -0.89952400 \\
\hline C & -0.05386200 & -2.69766000 & -0.74241400 \\
\hline $\mathrm{H}$ & -0.99455100 & -2.82013700 & -1.26887200 \\
\hline $\mathrm{H}$ & 0.74899900 & -2.79994600 & -1.47338600 \\
\hline $\mathrm{C}$ & 0.06200700 & -3.72675500 & 0.38410200 \\
\hline $\mathrm{H}$ & 0.04310900 & -4.72434600 & -0.06448000 \\
\hline $\mathrm{H}$ & 0.99089300 & -3.62675100 & 0.95108600 \\
\hline $\mathrm{H}$ & -0.78222400 & -3.64029200 & 1.06959600 \\
\hline
\end{tabular}

Table S6f. Cartesian coordinates for 5AB.

\begin{tabular}{lrrr} 
Atom & \multicolumn{3}{c}{ Coordinates $($ Angstroms) } \\
& X & \multicolumn{2}{c}{ Y } \\
\hline C & -1.94276600 & -1.36560100 & -0.18122200 \\
C & -0.75598100 & -0.64063600 & -0.11199300 \\
C & -0.83122500 & 0.76915000 & 0.11122300 \\
C & -2.09699200 & 1.38085500 & 0.23591000 \\
C & 1.54413300 & 0.88279500 & 0.10720300 \\
C & 1.62717800 & -0.53611400 & -0.05750300
\end{tabular}




\begin{tabular}{lrrr} 
C & 2.95692800 & -1.14609900 & 0.03966200 \\
$\mathrm{C}$ & 3.89160400 & 1.12270000 & 0.09011800 \\
$\mathrm{H}$ & -1.93697600 & -2.43373100 & -0.33404500 \\
$\mathrm{H}$ & -2.17891200 & 2.44608400 & 0.39043100 \\
$\mathrm{~N}$ & 4.03439900 & -0.25693800 & 0.11759100 \\
$\mathrm{~N}$ & 2.61357100 & 1.65750700 & 0.20259700 \\
$\mathrm{~N}$ & 0.48371800 & -1.27251400 & -0.25954300 \\
$\mathrm{~N}$ & 0.32827900 & 1.51769700 & 0.18685900 \\
$\mathrm{O}$ & 3.16489100 & -2.36018200 & 0.05893800 \\
$\mathrm{O}$ & 4.85396500 & 1.88198300 & -0.00615100 \\
$\mathrm{Cl}$ & -4.60348100 & -1.72181400 & -0.15098100 \\
$\mathrm{C}$ & -3.19662600 & -0.74218900 & -0.04718800 \\
$\mathrm{Cl}$ & -4.79193300 & 1.45976200 & 0.32359700 \\
$\mathrm{C}$ & -3.27244700 & 0.64558300 & 0.16143800 \\
$\mathrm{C}$ & 0.28238800 & 3.00339900 & 0.31901400 \\
$\mathrm{H}$ & -0.49457900 & 3.23903300 & 1.04535700 \\
$\mathrm{H}$ & 1.23475900 & 3.29965300 & 0.74786800 \\
$\mathrm{C}$ & 0.05859300 & 3.70002300 & -1.02519500 \\
$\mathrm{H}$ & 0.88458200 & 3.49150500 & -1.70972800 \\
$\mathrm{H}$ & -0.87775200 & 3.39735100 & -1.50075100 \\
$\mathrm{H}$ & 0.01994500 & 4.77871700 & -0.84923400 \\
$\mathrm{C}$ & 5.38535000 & -0.83796400 & 0.16420300 \\
$\mathrm{H}$ & 5.66315400 & -1.22546900 & -0.81934600 \\
$\mathrm{H}$ & 6.07788300 & -0.05350700 & 0.45819600 \\
$\mathrm{H}$ & 5.39616000 & -1.65181200 & 0.88789100 \\
$\mathrm{C}$ & 0.51754900 & -2.69568600 & -0.71154400 \\
$\mathrm{H}$ & -0.28198900 & -2.80105400 & -1.44566500 \\
$\mathrm{H}$ & 1.45979700 & -2.83860100 & -1.23017900 \\
$\mathrm{C}$ & 0.38417800 & -3.70367400 & 0.43192800 \\
$\mathrm{H}$ & 0.39183300 & -4.70901600 & 0.00060100 \\
$\mathrm{H}$ & 1.22736100 & -3.61611100 & 1.11862400 \\
$\mathrm{H}$ & -0.54503300 & -3.58207100 & 0.99437200 \\
---------------------------------------------------------------------------------------- \\
\hline
\end{tabular}

Table S6g. Cartesian coordinates for 6AB.

\begin{tabular}{|c|c|c|c|}
\hline \multirow[t]{2}{*}{ Atom } & \multicolumn{3}{|c|}{ Coordinates (Angstroms) } \\
\hline & $X$ & $\mathrm{Y}$ & $\mathrm{Z}$ \\
\hline $\mathrm{C}$ & -2.40771300 & -1.41654900 & -0.16578700 \\
\hline C & -1.22169600 & -0.67045800 & -0.10479900 \\
\hline C & -1.31905300 & 0.73548700 & 0.11961200 \\
\hline C & -2.59454800 & 1.33453000 & 0.19380000 \\
\hline C & 1.07281100 & 0.87679100 & 0.11276300 \\
\hline C & 1.15851900 & -0.53551200 & -0.07780300 \\
\hline C & 2.49726500 & -1.13008500 & -0.04653100 \\
\hline C & 3.40409100 & 1.15305800 & 0.11760700 \\
\hline $\mathrm{H}$ & -2.38144000 & -2.48966000 & -0.28197600 \\
\hline $\mathrm{H}$ & -2.67453900 & 2.40774300 & 0.29320800 \\
\hline $\mathrm{N}$ & 3.56245400 & -0.23165200 & 0.01424100 \\
\hline
\end{tabular}




$\begin{array}{lrrr}\text { N } & 2.12875500 & 1.66516100 & 0.19193500 \\ \mathrm{~N} & 0.01458600 & -1.28693600 & -0.24449000 \\ \mathrm{~N} & -0.16235000 & 1.49269800 & 0.22022900 \\ \mathrm{O} & 2.73070900 & -2.34515400 & -0.06322500 \\ \mathrm{O} & 4.38230400 & 1.90550800 & 0.15305500 \\ \mathrm{C} & -3.68091900 & -0.81645400 & -0.02052200 \\ \mathrm{C} & -3.77552600 & 0.61254500 & 0.10842900 \\ \mathrm{~N} & -4.78445300 & -1.58547700 & -0.00482600 \\ \mathrm{H} & -4.71958400 & -2.59248100 & -0.07609600 \\ \mathrm{H} & -5.68495200 & -1.21020100 & 0.26112900 \\ \mathrm{~N} & -5.02227400 & 1.19888800 & 0.22672800 \\ \mathrm{H} & -5.74710600 & 0.87621200 & -0.40545400 \\ \mathrm{H} & -5.02407000 & 2.20949300 & 0.29419400 \\ \mathrm{C} & 4.91321900 & -0.81058200 & 0.00957500 \\ \mathrm{H} & 5.62496100 & 0.00983800 & 0.01973100 \\ \mathrm{H} & 5.05116100 & -1.44118700 & 0.89050200 \\ \mathrm{H} & 5.04923100 & -1.41993300 & -0.88566200 \\ \mathrm{C} & -0.22204000 & 2.95875800 & 0.43908800 \\ \mathrm{H} & -1.03518700 & 3.15153000 & 1.13955500 \\ \mathrm{H} & 0.70671000 & 3.23404700 & 0.93142900 \\ \mathrm{C} & -0.38476000 & 3.74654500 & -0.86292000 \\ \mathrm{H} & 0.46956400 & 3.57674900 & -1.52342100 \\ \mathrm{H} & -1.29903300 & 3.47779800 & -1.39938100 \\ \mathrm{H} & -0.42959900 & 4.81386300 & -0.62624200 \\ \mathrm{C} & 0.06083800 & -2.72018000 & -0.66390000 \\ \mathrm{H} & -0.75658900 & -2.86334300 & -1.37036000 \\ \mathrm{H} & 0.99019400 & -2.86220100 & -1.20473600 \\ \mathrm{C} & -0.01830900 & -3.69649200 & 0.51109800 \\ \mathrm{H} & 0.84736700 & -3.57680000 & 1.16476000 \\ \mathrm{H} & -0.92908800 & -3.56544400 & 1.10178600 \\ \mathrm{H} & -0.01220700 & -4.71602400 & 0.11342300 \\ ------------------------------------------------------------------------------------------------------ \\ & & \end{array}$

Table S6h. Cartesian coordinates for 7AB.

\begin{tabular}{|c|c|c|c|}
\hline \multirow[t]{2}{*}{ Atom } & \multicolumn{3}{|c|}{ Coordinates (Angstroms) } \\
\hline & $\mathrm{X}$ & Y & $\mathrm{Z}$ \\
\hline $\mathrm{C}$ & -3.23095900 & -0.58746100 & 0.09938700 \\
\hline $\mathrm{C}$ & -1.99550700 & -1.24741400 & -0.08081400 \\
\hline $\mathrm{C}$ & -0.78092000 & -0.55479600 & -0.03688200 \\
\hline $\mathrm{C}$ & -0.80080500 & 0.85004000 & 0.20740400 \\
\hline $\mathrm{C}$ & -2.04855800 & 1.50247500 & 0.35974200 \\
\hline C & -3.25013500 & 0.82573100 & 0.30256100 \\
\hline $\mathrm{C}$ & 1.58195200 & 0.88618600 & 0.12928200 \\
\hline $\mathrm{C}$ & 1.60494200 & -0.52861300 & -0.06535100 \\
\hline $\mathrm{C}$ & 2.91758700 & -1.18219800 & -0.04922900 \\
\hline $\mathrm{C}$ & 3.93040600 & 1.05483800 & 0.06341500 \\
\hline $\mathrm{H}$ & -2.00691400 & -2.31433600 & -0.23161700 \\
\hline $\mathrm{H}$ & -2.10062800 & 2.56906600 & 0.52198100 \\
\hline
\end{tabular}




$\begin{array}{lrrr}\text { N } & 4.02473300 & -0.33261900 & -0.01740300 \\ \mathrm{~N} & 2.67633800 & 1.62666200 & 0.18344100 \\ \mathrm{~N} & 0.42972500 & -1.22755800 & -0.22288700 \\ \mathrm{~N} & 0.38506400 & 1.55703700 & 0.26788000 \\ \mathrm{O} & 3.09052800 & -2.40467300 & -0.05792700 \\ \mathrm{O} & 4.93043000 & 1.77431800 & 0.04489800 \\ \mathrm{O} & -4.40302900 & 1.49944600 & 0.53929800 \\ \mathrm{O} & -4.40405200 & -1.20526700 & 0.10782700 \\ \mathrm{C} & -4.50485900 & -2.63515600 & -0.08796700 \\ \mathrm{H} & -3.97463300 & -3.16258900 & 0.70833500 \\ \mathrm{H} & -5.56990600 & -2.84888900 & -0.03517500 \\ \mathrm{H} & -4.11305500 & -2.90873100 & -1.07059800 \\ \mathrm{C} & -5.35798900 & 1.59704400 & -0.54887600 \\ \mathrm{H} & -5.70703700 & 0.60937400 & -0.85443800 \\ \mathrm{H} & -6.18733900 & 2.18126600 & -0.15209900 \\ \mathrm{H} & -4.90035800 & 2.12187200 & -1.39257800 \\ \mathrm{C} & 0.39376800 & 3.03289400 & 0.46235000 \\ \mathrm{H} & -0.37103700 & 3.26792400 & 1.20214800 \\ \mathrm{H} & 1.35816100 & 3.27820800 & 0.89719100 \\ \mathrm{C} & 0.18768300 & 3.79752600 & -0.84702000 \\ \mathrm{H} & 0.99690500 & 3.58318900 & -1.54989600 \\ \mathrm{H} & -0.76530100 & 3.55456600 & -1.32447000 \\ \mathrm{H} & 0.19649400 & 4.86931900 & -0.62828800 \\ \mathrm{C} & 5.34889300 & -0.97199200 & -0.04933400 \\ \mathrm{H} & 5.44607200 & -1.57134300 & -0.95633000 \\ \mathrm{H} & 6.09753500 & -0.18514100 & -0.03640700 \\ \mathrm{H} & 5.46614100 & -1.62121500 & 0.82031900 \\ \mathrm{C} & 0.40369900 & -2.64959800 & -0.67778200 \\ \mathrm{H} & 1.32020400 & -2.82102500 & -1.23229300 \\ \mathrm{H} & -0.42595000 & -2.73350900 & -1.38010100 \\ \mathrm{C} & 0.28787200 & -3.65422500 & 0.47060800 \\ \mathrm{H} & -0.61333800 & -3.50382100 & 1.07075000 \\ \mathrm{H} & 0.24869900 & -4.66036300 & 0.04238800 \\ \mathrm{H} & 1.16081800 & -3.59075100 & 1.12222400 \\ ------------------------------------------------------------------------------------------------------- \\ \end{array}$

Table S6i. Cartesian coordinates for $8 \mathrm{AB}$.

\begin{tabular}{|c|c|c|c|}
\hline \multirow[t]{2}{*}{ Atom } & \multicolumn{3}{|c|}{ Coordinates (Angstroms) } \\
\hline & $\mathrm{X}$ & $\mathrm{Y}$ & $\mathrm{Z}$ \\
\hline C & 1.23643800 & -1.10444300 & -0.08405600 \\
\hline C & 0.00491500 & -0.45163000 & -0.06199200 \\
\hline C & -0.01000600 & 0.96518100 & 0.02393500 \\
\hline $\mathrm{C}$ & 1.22131600 & 1.65257100 & 0.05925900 \\
\hline C & -2.39738600 & 0.93090800 & 0.05082400 \\
\hline C & -2.37610200 & -0.50373800 & 0.01339800 \\
\hline $\mathrm{C}$ & -3.66866600 & -1.18952100 & 0.14215300 \\
\hline $\mathrm{C}$ & -4.74580700 & 1.02037800 & 0.05352400 \\
\hline $\mathrm{H}$ & 1.29314500 & -2.17851200 & -0.10293300 \\
\hline $\mathrm{H}$ & 1.22096400 & 2.73251400 & 0.09479900 \\
\hline
\end{tabular}




\begin{tabular}{|c|c|c|c|}
\hline $\mathrm{N}$ & -4.79774000 & -0.37362800 & 0.11946500 \\
\hline $\mathrm{N}$ & -3.50845400 & 1.63522500 & 0.05922400 \\
\hline $\mathrm{N}$ & -1.18934400 & -1.17884900 & -0.10637700 \\
\hline $\mathrm{N}$ & -1.21372300 & 1.64442700 & 0.07006300 \\
\hline O & -3.80093700 & -2.41023000 & 0.27345500 \\
\hline 0 & -5.77274700 & 1.69826000 & 0.00546400 \\
\hline $\mathrm{N}$ & 3.59381100 & 1.85300900 & -0.05458600 \\
\hline $\mathrm{H}$ & 3.40546900 & 2.75695700 & -0.47057200 \\
\hline $\mathrm{N}$ & 3.66070700 & -1.09594900 & -0.10425200 \\
\hline $\mathrm{H}$ & 4.45386900 & -0.58379200 & 0.32485500 \\
\hline $\mathrm{C}$ & 2.45511800 & 1.01575000 & 0.02594100 \\
\hline $\mathrm{C}$ & 2.47470200 & -0.40878500 & -0.06157700 \\
\hline $\mathrm{C}$ & 4.83120400 & 1.72773500 & 0.52215600 \\
\hline C & 3.88694900 & -2.40580500 & -0.54956200 \\
\hline $\mathrm{C}$ & 5.28830200 & -2.89986700 & -0.30157900 \\
\hline $\mathrm{H}$ & 5.43578100 & -3.06971200 & 0.77082400 \\
\hline $\mathrm{H}$ & 5.43455900 & -3.83717800 & -0.83698500 \\
\hline $\mathrm{H}$ & 6.03069200 & -2.16532500 & -0.62779700 \\
\hline $\mathrm{C}$ & 5.75454100 & 2.90959800 & 0.36801800 \\
\hline $\mathrm{H}$ & 6.66872700 & 2.57291900 & -0.12858000 \\
\hline $\mathrm{H}$ & 5.32214000 & 3.73663300 & -0.19695300 \\
\hline $\mathrm{H}$ & 6.03004400 & 3.26154600 & 1.36633300 \\
\hline 0 & 5.18478000 & 0.70604800 & 1.12917800 \\
\hline 0 & 3.01250400 & -3.06887000 & -1.09376000 \\
\hline $\mathrm{C}$ & -6.10063600 & -1.05010100 & 0.20757400 \\
\hline $\mathrm{H}$ & -6.87399700 & -0.29198700 & 0.12498300 \\
\hline $\mathrm{H}$ & -6.19204900 & -1.77573100 & -0.60232900 \\
\hline $\mathrm{H}$ & -6.18321700 & -1.57213500 & 1.16315000 \\
\hline $\mathrm{C}$ & -1.26337100 & 3.12912700 & 0.12265100 \\
\hline $\mathrm{H}$ & -2.21453600 & 3.38646500 & 0.58026000 \\
\hline $\mathrm{H}$ & -0.47480100 & 3.45956300 & 0.79911400 \\
\hline C & -1.14716900 & 3.76928200 & -1.26209300 \\
\hline $\mathrm{H}$ & -0.21399000 & 3.50281800 & -1.76579200 \\
\hline $\mathrm{H}$ & -1.98417100 & 3.46659900 & -1.89650900 \\
\hline $\mathrm{H}$ & -1.17601400 & 4.85699100 & -1.14918400 \\
\hline $\mathrm{C}$ & -1.12614600 & -2.64650900 & -0.38304700 \\
\hline $\mathrm{H}$ & -0.31569900 & -2.79174800 & -1.09711400 \\
\hline $\mathrm{H}$ & -2.05302900 & -2.91450500 & -0.87842200 \\
\hline $\mathrm{C}$ & -0.93895500 & -3.49165500 & 0.87812900 \\
\hline $\mathrm{H}$ & -0.89020700 & -4.54326300 & 0.57988000 \\
\hline $\mathrm{H}$ & -0.01808400 & -3.24687100 & 1.41412300 \\
\hline $\mathrm{H}$ & -1.78665200 & -3.36440800 & 1.55344400 \\
\hline
\end{tabular}

Table S6j. Cartesian coordinates for 9AB.

\begin{tabular}{cc} 
Atom & \multicolumn{2}{c}{ Coordinates (Angstroms) } \\
X & Y \\
S50
\end{tabular}




\begin{tabular}{|c|c|c|c|}
\hline $\mathrm{C}$ & -3.20687500 & -0.72907000 & 0.01379000 \\
\hline C & -1.93349100 & -1.34919600 & -0.08027200 \\
\hline C & -0.73573900 & -0.63697900 & -0.07085500 \\
\hline C & -0.80308900 & 0.77875800 & 0.04025000 \\
\hline C & -2.07856600 & 1.39463500 & 0.11375600 \\
\hline C & -3.27581900 & 0.67777000 & 0.10655800 \\
\hline C & 1.57596300 & 0.88687200 & 0.03350000 \\
\hline $\mathrm{C}$ & 1.64967400 & -0.54142400 & -0.02326500 \\
\hline C & 2.97591200 & -1.14661200 & 0.11374900 \\
\hline C & 3.92124900 & 1.12091200 & 0.03547900 \\
\hline $\mathrm{H}$ & -1.91922800 & -2.42753000 & -0.15099500 \\
\hline $\mathrm{H}$ & -2.14045700 & 2.47221800 & 0.17558700 \\
\hline $\mathrm{N}$ & 4.05674900 & -0.25927000 & 0.13390600 \\
\hline $\mathrm{N}$ & 2.64474100 & 1.66325100 & 0.06483600 \\
\hline $\mathrm{N}$ & 0.50310500 & -1.28640200 & -0.16747700 \\
\hline $\mathrm{N}$ & 0.35636600 & 1.52939400 & 0.05939200 \\
\hline O & 3.18431300 & -2.35869500 & 0.21497400 \\
\hline O & 4.89586600 & 1.86726900 & -0.05569300 \\
\hline C & -4.42225700 & -1.61192800 & -0.03891500 \\
\hline $\mathrm{H}$ & -4.19007800 & -2.56528300 & 0.44765900 \\
\hline $\mathrm{H}$ & -5.24755700 & -1.15997500 & 0.51568000 \\
\hline C & -4.58167800 & 1.43132400 & 0.23329900 \\
\hline $\mathrm{H}$ & -5.36645000 & 0.92337000 & -0.33407800 \\
\hline $\mathrm{H}$ & -4.46079000 & 2.41927300 & -0.22264300 \\
\hline C & -5.03040900 & 1.60431400 & 1.69875700 \\
\hline $\mathrm{H}$ & -5.17985000 & 0.63776600 & 2.19010500 \\
\hline $\mathrm{H}$ & -4.28409800 & 2.16267500 & 2.27268000 \\
\hline $\mathrm{H}$ & -5.97462900 & 2.15579200 & 1.73818500 \\
\hline C & -4.87772300 & -1.89565400 & -1.49288600 \\
\hline $\mathrm{H}$ & -5.75078500 & -2.55435400 & -1.47724300 \\
\hline $\mathrm{H}$ & -5.15093600 & -0.97100400 & -2.00955900 \\
\hline $\mathrm{H}$ & -4.08574900 & -2.38725600 & -2.06539200 \\
\hline C & 0.31648600 & 3.01856800 & 0.07787200 \\
\hline $\mathrm{H}$ & -0.47231600 & 3.31474900 & 0.76827600 \\
\hline $\mathrm{H}$ & 1.26299300 & 3.34407600 & 0.49886000 \\
\hline C & 0.11855400 & 3.61189600 & -1.31916000 \\
\hline $\mathrm{H}$ & 0.95276300 & 3.34507100 & -1.97312900 \\
\hline $\mathrm{H}$ & -0.81285900 & 3.27648100 & -1.78310700 \\
\hline $\mathrm{H}$ & 0.08432600 & 4.70163300 & -1.23089200 \\
\hline C & 5.39991200 & -0.85013800 & 0.23374800 \\
\hline $\mathrm{H}$ & 6.11140900 & -0.04013400 & 0.36786000 \\
\hline $\mathrm{H}$ & 5.43144700 & -1.53236000 & 1.08358500 \\
\hline $\mathrm{H}$ & 5.63424000 & -1.40425100 & -0.67827700 \\
\hline C & 0.53011400 & -2.73758500 & -0.51096600 \\
\hline $\mathrm{H}$ & -0.27567700 & -2.89758500 & -1.22802200 \\
\hline $\mathrm{H}$ & 1.46808200 & -2.92384700 & -1.02417200 \\
\hline C & 0.40228400 & -3.65727000 & 0.70521100 \\
\hline $\mathrm{H}$ & -0.52169000 & -3.48627600 & 1.26371300 \\
\hline $\mathrm{H}$ & 0.40078100 & -4.69301400 & 0.35222200 \\
\hline $\mathrm{H}$ & 1.25193100 & -3.52280000 & 1.37636500 \\
\hline
\end{tabular}


Table S6k. Cartesian coordinates for 1A.

\begin{tabular}{|c|c|c|c|}
\hline \multirow[t]{2}{*}{ Atom } & \multicolumn{3}{|c|}{ Coordinates (Angstroms) } \\
\hline & $\mathrm{X}$ & $\mathrm{Y}$ & $\mathrm{Z}$ \\
\hline $\mathrm{C}$ & 2.83448500 & 1.35192000 & -0.12871800 \\
\hline $\mathrm{C}$ & 1.49425500 & 1.77988200 & -0.23887600 \\
\hline $\mathrm{C}$ & 0.40654100 & 0.91041800 & -0.15854000 \\
\hline $\mathrm{C}$ & 0.66207300 & -0.47445200 & 0.05400800 \\
\hline $\mathrm{C}$ & 2.00091700 & -0.91294300 & 0.15612400 \\
\hline $\mathrm{C}$ & 3.06507300 & -0.02813700 & 0.06974100 \\
\hline $\mathrm{C}$ & -1.67433200 & -0.89784400 & 0.09501300 \\
\hline $\mathrm{C}$ & -1.94432300 & 0.49887200 & -0.06776600 \\
\hline $\mathrm{C}$ & -3.34130500 & 0.93100500 & 0.04890200 \\
\hline $\mathrm{C}$ & -3.97115500 & -1.43978200 & 0.12972500 \\
\hline $\mathrm{H}$ & 1.33229200 & 2.83707400 & -0.38539300 \\
\hline $\mathrm{H}$ & 2.21302000 & -1.96042500 & 0.30359900 \\
\hline $\mathrm{N}$ & -4.29163800 & -0.09113900 & 0.15972500 \\
\hline $\mathrm{N}$ & -2.63396400 & -1.80455500 & 0.22026800 \\
\hline $\mathrm{N}$ & -0.90769800 & 1.37653300 & -0.28643100 \\
\hline $\mathrm{N}$ & -0.38880100 & -1.36951600 & 0.14628000 \\
\hline 0 & -3.70700300 & 2.10701400 & 0.05827100 \\
\hline 0 & -4.82880900 & -2.31748500 & 0.04966200 \\
\hline $\mathrm{C}$ & 4.47036100 & -0.57262500 & 0.20444600 \\
\hline $\mathrm{C}$ & 3.93001100 & 2.36860200 & -0.24706000 \\
\hline $\mathrm{C}$ & -0.14500800 & -2.83751000 & 0.26010500 \\
\hline $\mathrm{H}$ & 0.66489400 & -2.97581700 & 0.97555400 \\
\hline $\mathrm{H}$ & -1.04492900 & -3.26221400 & 0.69437100 \\
\hline C & 0.15395400 & -3.48282000 & -1.09514800 \\
\hline $\mathrm{H}$ & -0.69787900 & -3.37198500 & -1.77093700 \\
\hline $\mathrm{H}$ & 1.04020000 & -3.05787700 & -1.57351000 \\
\hline $\mathrm{H}$ & 0.33042200 & -4.55004900 & -0.93430700 \\
\hline $\mathrm{C}$ & -5.70759100 & 0.30224000 & 0.22838000 \\
\hline $\mathrm{H}$ & -6.07665500 & 0.56481100 & -0.76672700 \\
\hline $\mathrm{H}$ & -6.27315400 & -0.54179900 & 0.61559100 \\
\hline $\mathrm{H}$ & -5.80142300 & 1.16354700 & 0.88735800 \\
\hline C & -1.13323300 & 2.78595200 & -0.72539700 \\
\hline $\mathrm{H}$ & -0.36120900 & 3.00313100 & -1.46387900 \\
\hline $\mathrm{H}$ & -2.09071000 & 2.80751700 & -1.23513300 \\
\hline C & -1.12483900 & 3.79233500 & 0.42719800 \\
\hline $\mathrm{H}$ & -1.26645000 & 4.79230700 & 0.00661100 \\
\hline $\mathrm{H}$ & -1.94480300 & 3.58892000 & 1.11735200 \\
\hline $\mathrm{H}$ & -0.18385900 & 3.78657600 & 0.98283800 \\
\hline $\mathrm{H}$ & 4.65359500 & 2.27842700 & 0.56829500 \\
\hline $\mathrm{H}$ & 3.52403200 & 3.38065600 & -0.24609400 \\
\hline $\mathrm{H}$ & 4.48514400 & 2.22336500 & -1.18175700 \\
\hline $\mathrm{F}$ & 5.10061300 & -0.07308400 & 1.29915700 \\
\hline $\mathrm{F}$ & 4.48985400 & -1.91830700 & 0.32245600 \\
\hline $\mathrm{F}$ & 5.24101700 & -0.25886800 & -0.86705900 \\
\hline
\end{tabular}


Table S6l. Cartesian coordinates for 1B.

\begin{tabular}{|c|c|c|c|}
\hline \multirow[t]{2}{*}{ Atom } & \multicolumn{3}{|c|}{ Coordinates (Angstroms) } \\
\hline & $\mathrm{X}$ & $\mathrm{Y}$ & $\mathrm{Z}$ \\
\hline C & -3.02612300 & -0.07577500 & 0.01283300 \\
\hline $\mathrm{C}$ & -1.88955100 & -0.87907800 & -0.12551800 \\
\hline $\mathrm{C}$ & -0.60162600 & -0.34146000 & -0.06516000 \\
\hline $\mathrm{C}$ & -0.47488000 & 1.06124700 & 0.15758800 \\
\hline $\mathrm{C}$ & -1.63576200 & 1.85008200 & 0.27529100 \\
\hline $\mathrm{C}$ & -2.92441100 & 1.31641100 & 0.21221500 \\
\hline $\mathrm{C}$ & 1.88879300 & 0.83036600 & 0.15210200 \\
\hline $\mathrm{C}$ & 1.77610100 & -0.57930400 & -0.03891200 \\
\hline $\mathrm{C}$ & 3.00673100 & -1.36947600 & 0.02458000 \\
\hline $\mathrm{C}$ & 4.24574700 & 0.74611300 & 0.07567600 \\
\hline $\mathrm{H}$ & -2.03139200 & -1.93760700 & -0.27405200 \\
\hline $\mathrm{H}$ & -1.54431600 & 2.91634900 & 0.41943200 \\
\hline $\mathrm{N}$ & 4.20324900 & -0.63452500 & 0.09307400 \\
\hline $\mathrm{N}$ & 3.06777800 & 1.43983100 & 0.30047700 \\
\hline $\mathrm{N}$ & 0.53025900 & -1.14189000 & -0.23216000 \\
\hline $\mathrm{N}$ & 0.78833300 & 1.63164700 & 0.23913500 \\
\hline 0 & 3.05039900 & -2.59937400 & 0.01736100 \\
\hline 0 & 5.28629800 & 1.38011100 & -0.10154200 \\
\hline $\mathrm{C}$ & -4.11260600 & 2.23171500 & 0.36048800 \\
\hline $\mathrm{C}$ & -4.38320200 & -0.74837000 & -0.06464900 \\
\hline $\mathrm{F}$ & -5.11084400 & -0.53800400 & 1.05995800 \\
\hline $\mathrm{F}$ & -4.28814900 & -2.08615400 & -0.22639800 \\
\hline $\mathrm{F}$ & -5.11680400 & -0.27815100 & -1.10454100 \\
\hline $\mathrm{C}$ & 0.95971900 & 3.10874100 & 0.36755000 \\
\hline $\mathrm{H}$ & 0.21882700 & 3.45592800 & 1.08608700 \\
\hline $\mathrm{H}$ & 1.94468000 & 3.26241900 & 0.79953500 \\
\hline $\mathrm{C}$ & 0.84909600 & 3.82632800 & -0.98024400 \\
\hline $\mathrm{H}$ & 1.63611500 & 3.49291800 & -1.66140200 \\
\hline $\mathrm{H}$ & -0.12162600 & 3.66751100 & -1.45664800 \\
\hline $\mathrm{H}$ & 0.97456000 & 4.89856600 & -0.80599000 \\
\hline C & 5.47210200 & -1.37754900 & 0.07765300 \\
\hline $\mathrm{H}$ & 5.74756800 & -1.63682800 & -0.94834100 \\
\hline $\mathrm{H}$ & 6.24279700 & -0.74067300 & 0.50792400 \\
\hline $\mathrm{H}$ & 5.35096500 & -2.28617600 & 0.66349700 \\
\hline $\mathrm{C}$ & 0.35684300 & -2.55127400 & -0.69029300 \\
\hline $\mathrm{H}$ & -0.46712300 & -2.54235000 & -1.40428700 \\
\hline $\mathrm{H}$ & 1.25773200 & -2.81996500 & -1.23227100 \\
\hline $\mathrm{C}$ & 0.11138900 & -3.53735600 & 0.45400800 \\
\hline $\mathrm{H}$ & -0.02705500 & -4.53269700 & 0.02165900 \\
\hline $\mathrm{H}$ & 0.97220800 & -3.56845400 & 1.12341300 \\
\hline $\mathrm{H}$ & -0.78097900 & -3.29184600 & 1.03542800 \\
\hline $\mathrm{H}$ & -4.68901600 & 1.98324200 & 1.25675900 \\
\hline $\mathrm{H}$ & -3.78573600 & 3.26930500 & 0.44294400 \\
\hline
\end{tabular}


$-0.49633400$

Table S6m. Cartesian coordinates for 2A.

\begin{tabular}{|c|c|c|c|}
\hline \multirow[t]{2}{*}{ Atom } & \multicolumn{3}{|c|}{ Coordinates (Angstroms) } \\
\hline & $\mathrm{X}$ & $\mathrm{Y}$ & $\mathrm{Z}$ \\
\hline $\mathrm{C}$ & -1.83135500 & 1.68480900 & 0.23910400 \\
\hline $\mathrm{C}$ & -0.71604800 & 0.85307000 & 0.14643400 \\
\hline $\mathrm{C}$ & -0.92759100 & -0.54214800 & -0.08051200 \\
\hline $\mathrm{C}$ & -2.24492000 & -1.01937200 & -0.17813800 \\
\hline $\mathrm{C}$ & 1.41953700 & -0.88920700 & -0.11419200 \\
\hline $\mathrm{C}$ & 1.64470500 & 0.51547300 & 0.06560500 \\
\hline $\mathrm{C}$ & 3.03039700 & 0.99115500 & -0.03859900 \\
\hline $\mathrm{C}$ & 3.73268300 & -1.35903500 & -0.10845700 \\
\hline $\mathrm{H}$ & -1.69973600 & 2.74275900 & 0.40455600 \\
\hline $\mathrm{H}$ & -2.45161900 & -2.06826300 & -0.32280900 \\
\hline $\mathrm{N}$ & 4.01252900 & -0.00153100 & -0.13226900 \\
\hline $\mathrm{N}$ & 2.40835300 & -1.76243400 & -0.23995100 \\
\hline $\mathrm{N}$ & 0.57901700 & 1.35674000 & 0.27727700 \\
\hline $\mathrm{N}$ & 0.15180900 & -1.39971100 & -0.18569400 \\
\hline O & 3.35918400 & 2.17680900 & -0.05018800 \\
\hline 0 & 4.60938600 & -2.21295400 & -0.00132600 \\
\hline $\mathrm{C}$ & -3.16341800 & 1.23160800 & 0.11789500 \\
\hline $\mathrm{C}$ & -3.32901800 & -0.15626700 & -0.09368900 \\
\hline $\mathrm{C}$ & -0.04497700 & -2.87228900 & -0.33658900 \\
\hline $\mathrm{H}$ & -0.85786200 & -3.01501700 & -1.04781000 \\
\hline $\mathrm{H}$ & 0.86433100 & -3.25492400 & -0.79036300 \\
\hline $\mathrm{C}$ & -0.31006400 & -3.56318400 & 1.00320100 \\
\hline $\mathrm{H}$ & 0.54489500 & -3.44601900 & 1.67371200 \\
\hline $\mathrm{H}$ & -1.20402300 & -3.17874000 & 1.50048200 \\
\hline $\mathrm{H}$ & -0.45629300 & -4.62999900 & 0.81279100 \\
\hline $\mathrm{C}$ & 0.75977900 & 2.77856800 & 0.70565900 \\
\hline $\mathrm{H}$ & -0.01592300 & 2.97494800 & 1.44573500 \\
\hline $\mathrm{H}$ & 1.71769000 & 2.83447400 & 1.21116700 \\
\hline $\mathrm{C}$ & 0.71319000 & 3.77069100 & -0.45769000 \\
\hline $\mathrm{H}$ & 1.53860000 & 3.58954200 & -1.14764300 \\
\hline $\mathrm{H}$ & -0.22821300 & 3.72496200 & -1.01072300 \\
\hline $\mathrm{H}$ & 0.81972600 & 4.77922800 & -0.04769100 \\
\hline $\mathrm{C}$ & 5.41648000 & 0.43750000 & -0.17714000 \\
\hline $\mathrm{H}$ & 6.02075600 & -0.40324000 & -0.50870100 \\
\hline $\mathrm{H}$ & 5.50304200 & 1.27160500 & -0.87158300 \\
\hline $\mathrm{H}$ & 5.74378400 & 0.75629900 & 0.81588300 \\
\hline $\mathrm{N}$ & -4.65075000 & -0.77496300 & -0.22749500 \\
\hline 0 & -5.62124600 & -0.04467500 & -0.44190100 \\
\hline 0 & -4.72236900 & -2.00459000 & -0.12875000 \\
\hline $\mathrm{C}$ & -4.26303000 & 2.23894200 & 0.24295700 \\
\hline $\mathrm{H}$ & -4.79819500 & 2.34313000 & -0.70698100 \\
\hline $\mathrm{H}$ & -5.00932900 & 1.92507200 & 0.97856000 \\
\hline
\end{tabular}


0.53057800

Table S6n. Cartesian coordinates for 2B.

\begin{tabular}{|c|c|c|c|}
\hline \multirow[t]{2}{*}{ Atom } & \multicolumn{3}{|c|}{ Coordinates (Angstroms) } \\
\hline & $X$ & $\mathrm{Y}$ & $\mathrm{Z}$ \\
\hline C & 2.12010700 & -1.01889200 & 0.13092000 \\
\hline C & 0.86104900 & -0.43251300 & 0.07240300 \\
\hline $\mathrm{C}$ & 0.79342700 & 0.98092100 & -0.12902300 \\
\hline C & 1.98568400 & 1.72692100 & -0.21400400 \\
\hline $\mathrm{C}$ & -1.57843300 & 0.84221500 & -0.14669700 \\
\hline C & -1.52216300 & -0.57572000 & 0.03135800 \\
\hline C & -2.78557000 & -1.31357100 & -0.03924600 \\
\hline C & -3.93531200 & 0.85354600 & -0.07898600 \\
\hline $\mathrm{H}$ & 2.24609200 & -2.08125100 & 0.26843700 \\
\hline $\mathrm{H}$ & 1.93554700 & 2.80052200 & -0.31744900 \\
\hline $\mathrm{N}$ & -3.94852400 & -0.52842900 & -0.10663800 \\
\hline $\mathrm{N}$ & -2.72495700 & 1.50022300 & -0.28558800 \\
\hline $\mathrm{N}$ & -0.30472100 & -1.18920900 & 0.22316200 \\
\hline $\mathrm{N}$ & -0.44095000 & 1.60043700 & -0.22112900 \\
\hline O & -2.87808700 & -2.54042000 & -0.04045100 \\
\hline O & -4.95103200 & 1.52608900 & 0.09096000 \\
\hline C & 3.27927200 & -0.24336400 & -0.00289600 \\
\hline C & 3.26070100 & 1.15007100 & -0.16774500 \\
\hline $\mathrm{N}$ & 4.54513400 & -0.98660900 & 0.06714700 \\
\hline C & -0.55575400 & 3.08340800 & -0.35632700 \\
\hline $\mathrm{H}$ & 0.21550200 & 3.40147400 & -1.05596500 \\
\hline $\mathrm{H}$ & -1.52278700 & 3.26982900 & -0.81523100 \\
\hline C & -0.45709300 & 3.80107700 & 0.99218500 \\
\hline $\mathrm{H}$ & -1.27481000 & 3.49974900 & 1.65158700 \\
\hline $\mathrm{H}$ & 0.49319900 & 3.60952700 & 1.49683000 \\
\hline $\mathrm{H}$ & -0.53839100 & 4.87629100 & 0.81041400 \\
\hline C & -0.18444000 & -2.61109900 & 0.66072500 \\
\hline $\mathrm{H}$ & 0.63782600 & -2.64158800 & 1.37633500 \\
\hline $\mathrm{H}$ & -1.09609200 & -2.85448000 & 1.19661900 \\
\hline C & 0.02868300 & -3.58701000 & -0.49842000 \\
\hline $\mathrm{H}$ & -0.83380800 & -3.58235400 & -1.16650500 \\
\hline $\mathrm{H}$ & 0.92694500 & -3.35978900 & -1.07823700 \\
\hline $\mathrm{H}$ & 0.13789500 & -4.59205700 & -0.08060600 \\
\hline C & -5.24807600 & -1.21707100 & -0.10617200 \\
\hline $\mathrm{H}$ & -5.98466900 & -0.55075000 & -0.55130000 \\
\hline $\mathrm{H}$ & -5.15659600 & -2.13352700 & -0.68494600 \\
\hline $\mathrm{H}$ & -5.54881800 & -1.45763600 & 0.91725600 \\
\hline O & 5.51453600 & -0.53776200 & -0.54362500 \\
\hline 0 & 4.55713300 & -2.02817400 & 0.72756600 \\
\hline $\mathrm{C}$ & 4.47062400 & 2.03867700 & -0.26433900 \\
\hline $\mathrm{H}$ & 5.21732900 & 1.78809900 & 0.49276300 \\
\hline $\mathrm{H}$ & 4.95229200 & 1.92840300 & -1.24016500 \\
\hline
\end{tabular}


$-0.13654100$

Table S6o. Cartesian coordinates for 3A.

\begin{tabular}{|c|c|c|c|}
\hline \multirow[t]{2}{*}{ Atom } & \multicolumn{3}{|c|}{ Coordinates (Angstroms) } \\
\hline & $X$ & $\mathrm{Y}$ & $\mathrm{Z}$ \\
\hline $\mathrm{C}$ & -3.40431200 & 1.20593100 & 0.06850400 \\
\hline C & -2.08025800 & 1.68169300 & 0.18869200 \\
\hline $\mathrm{C}$ & -0.96423900 & 0.84627100 & 0.12183500 \\
\hline C & -1.17266800 & -0.55185700 & -0.09318100 \\
\hline $\mathrm{C}$ & -2.49202600 & -1.03078400 & -0.19673400 \\
\hline C & -3.59620500 & -0.18380800 & -0.12100900 \\
\hline C & 1.17999400 & -0.89345900 & -0.10695300 \\
\hline C & 1.39952700 & 0.51203700 & 0.06589000 \\
\hline C & 2.78426500 & 0.98923800 & -0.02138900 \\
\hline $\mathrm{C}$ & 3.49414100 & -1.36021100 & -0.10601600 \\
\hline $\mathrm{H}$ & -1.95194100 & 2.74415300 & 0.32969300 \\
\hline $\mathrm{H}$ & -2.69250900 & -2.08382200 & -0.33754500 \\
\hline $\mathrm{N}$ & 3.76976800 & 0.00000200 & -0.10659000 \\
\hline $\mathrm{N}$ & 2.16951900 & -1.76691900 & -0.21382900 \\
\hline $\mathrm{N}$ & 0.32999400 & 1.35338300 & 0.26196600 \\
\hline $\mathrm{N}$ & -0.08916600 & -1.40661800 & -0.18121000 \\
\hline 0 & 3.11362100 & 2.17613000 & -0.02703000 \\
\hline O & 4.37864200 & -2.21100600 & -0.03509800 \\
\hline C & -0.28114900 & -2.87966500 & -0.32121400 \\
\hline $\mathrm{H}$ & -1.09220600 & -3.03191200 & -1.03226500 \\
\hline $\mathrm{H}$ & 0.62993600 & -3.26477500 & -0.76907200 \\
\hline C & -0.54896500 & -3.56002500 & 1.02336300 \\
\hline $\mathrm{H}$ & 0.30314000 & -3.43410400 & 1.69608700 \\
\hline $\mathrm{H}$ & -1.44557000 & -3.17235600 & 1.51361600 \\
\hline $\mathrm{H}$ & -0.69207800 & -4.62928700 & 0.84383200 \\
\hline $\mathrm{C}$ & 5.16982100 & 0.45149300 & -0.14372300 \\
\hline $\mathrm{H}$ & 5.46263500 & 0.84696900 & 0.83203300 \\
\hline $\mathrm{H}$ & 5.79062500 & -0.40408300 & -0.39561300 \\
\hline $\mathrm{H}$ & 5.27211800 & 1.23478800 & -0.89408600 \\
\hline $\mathrm{C}$ & 0.50423100 & 2.77453400 & 0.69178000 \\
\hline $\mathrm{H}$ & 1.45260100 & 2.83051800 & 1.21536600 \\
\hline $\mathrm{H}$ & -0.28551400 & 2.97365400 & 1.41610100 \\
\hline C & 0.48150200 & 3.76743200 & -0.47180400 \\
\hline $\mathrm{H}$ & 0.58258200 & 4.77603900 & -0.06028900 \\
\hline $\mathrm{H}$ & 1.31913100 & 3.58450300 & -1.14635800 \\
\hline $\mathrm{H}$ & -0.44969500 & 3.72343700 & -1.04203100 \\
\hline C & -4.94685400 & -0.78771900 & -0.24391300 \\
\hline $\mathrm{H}$ & -5.80390500 & -0.09913800 & -0.18484600 \\
\hline O & -5.12781500 & -1.98702500 & -0.40329500 \\
\hline C & -4.53682200 & 2.18631500 & 0.14988300 \\
\hline $\mathrm{H}$ & -5.14624000 & 2.16276900 & -0.76092900 \\
\hline $\mathrm{H}$ & -5.20355900 & 1.95153000 & 0.98776200 \\
\hline
\end{tabular}


Table S6p. Cartesian coordinates for 3B.

\begin{tabular}{|c|c|c|c|}
\hline \multirow[t]{2}{*}{ Atom } & \multicolumn{3}{|c|}{ Coordinates (Angstroms) } \\
\hline & $X$ & $\mathrm{Y}$ & $\mathrm{Z}$ \\
\hline $\mathrm{C}$ & 3.54349200 & -0.34342600 & 0.00010300 \\
\hline C & 2.34227500 & -1.07757200 & 0.12853700 \\
\hline $\mathrm{C}$ & 1.09637400 & -0.47027500 & 0.07770300 \\
\hline C & 1.05179800 & 0.94274500 & -0.13606000 \\
\hline $\mathrm{C}$ & 2.26387100 & 1.66527300 & -0.24181500 \\
\hline C & 3.51941500 & 1.05252200 & -0.18043800 \\
\hline C & -1.32550200 & 0.85063600 & -0.13136600 \\
\hline C & -1.29206600 & -0.56862000 & 0.05070600 \\
\hline C & -2.57055600 & -1.28151100 & -0.00711800 \\
\hline C & -3.68155400 & 0.90772500 & -0.07229400 \\
\hline $\mathrm{H}$ & 2.44765400 & -2.14465100 & 0.26368200 \\
\hline $\mathrm{H}$ & 2.23317800 & 2.73706900 & -0.37189800 \\
\hline $\mathrm{N}$ & -3.71683900 & -0.47678600 & -0.08295500 \\
\hline $\mathrm{N}$ & -2.45212500 & 1.53576900 & -0.23570600 \\
\hline $\mathrm{N}$ & -0.08792200 & -1.20529100 & 0.23493100 \\
\hline $\mathrm{N}$ & -0.16520800 & 1.58607100 & -0.22582000 \\
\hline 0 & -2.68610300 & -2.50780200 & 0.00400500 \\
\hline O & -4.69590500 & 1.59193000 & 0.04946200 \\
\hline C & 4.81685000 & -1.10735000 & 0.06446300 \\
\hline $\mathrm{H}$ & 5.74763200 & -0.52840700 & -0.03255100 \\
\hline O & 4.85272500 & -2.32058500 & 0.21438800 \\
\hline C & -0.25264600 & 3.06740700 & -0.39180000 \\
\hline $\mathrm{H}$ & 0.52122000 & 3.35759400 & -1.10060100 \\
\hline $\mathrm{H}$ & -1.21620400 & 3.26395400 & -0.85260400 \\
\hline C & -0.13494800 & 3.81363200 & 0.93921300 \\
\hline $\mathrm{H}$ & -0.95768600 & 3.54639900 & 1.60696400 \\
\hline $\mathrm{H}$ & 0.81171000 & 3.61180600 & 1.44689700 \\
\hline $\mathrm{H}$ & -0.19182900 & 4.88643600 & 0.73430700 \\
\hline C & -5.02599200 & -1.14723100 & -0.08678400 \\
\hline $\mathrm{H}$ & -5.32836000 & -1.39466700 & 0.93456900 \\
\hline $\mathrm{H}$ & -5.75331500 & -0.46729900 & -0.52498600 \\
\hline $\mathrm{H}$ & -4.94889600 & -2.06029800 & -0.67335500 \\
\hline C & 0.00891600 & -2.62844900 & 0.67044000 \\
\hline $\mathrm{H}$ & -0.90334900 & -2.85660200 & 1.21194900 \\
\hline $\mathrm{H}$ & 0.83643600 & -2.67559000 & 1.37874300 \\
\hline $\mathrm{C}$ & 0.19607300 & -3.60720500 & -0.49089400 \\
\hline $\mathrm{H}$ & 0.28740200 & -4.61530900 & -0.07588600 \\
\hline $\mathrm{H}$ & -0.66967900 & -3.58435900 & -1.15467300 \\
\hline $\mathrm{H}$ & 1.09599600 & -3.39596900 & -1.07419800 \\
\hline $\mathrm{C}$ & 4.76368700 & 1.89539600 & -0.30546800 \\
\hline $\mathrm{H}$ & 5.38801100 & 1.81928300 & 0.59014400 \\
\hline $\mathrm{H}$ & 5.37088000 & 1.58618600 & -1.16202500 \\
\hline
\end{tabular}


$-0.44322600$

Table S6q. Cartesian coordinates for 4A.

\begin{tabular}{|c|c|c|c|}
\hline \multirow[t]{2}{*}{ Atom } & \multicolumn{3}{|c|}{ Coordinates (Angstroms) } \\
\hline & $X$ & $\mathrm{Y}$ & $\mathrm{Z}$ \\
\hline $\mathrm{C}$ & 2.43020800 & -1.35853200 & -0.16806300 \\
\hline $\mathrm{C}$ & 1.23453900 & -0.63922900 & -0.09657600 \\
\hline $\mathrm{C}$ & 1.29560400 & 0.76888300 & 0.14211000 \\
\hline $\mathrm{C}$ & 2.55886700 & 1.38772800 & 0.28070900 \\
\hline $\mathrm{C}$ & -1.07860100 & 0.86948800 & 0.12486800 \\
\hline $\mathrm{C}$ & -1.15427900 & -0.54379400 & -0.05929600 \\
\hline C & -2.48070500 & -1.15987700 & 0.01949700 \\
\hline $\mathrm{C}$ & -3.42644900 & 1.10447000 & 0.08932000 \\
\hline $\mathrm{H}$ & 2.41375400 & -2.42539600 & -0.33367200 \\
\hline $\mathrm{H}$ & 2.66085800 & 2.44985500 & 0.44796000 \\
\hline $\mathrm{N}$ & -3.56415200 & -0.27486700 & 0.09887400 \\
\hline $\mathrm{N}$ & -2.15418600 & 1.64149800 & 0.23148000 \\
\hline $\mathrm{N}$ & -0.00282000 & -1.27373600 & -0.26038000 \\
\hline $\mathrm{N}$ & 0.13147800 & 1.50987200 & 0.22028500 \\
\hline O & -2.68717500 & -2.37486700 & 0.02095000 \\
\hline 0 & -4.39051900 & 1.86225500 & -0.01640400 \\
\hline $\mathrm{C}$ & 3.69590700 & -0.75638500 & -0.02482100 \\
\hline $\mathrm{C}$ & 3.70535600 & 0.62597300 & 0.19741900 \\
\hline $\mathrm{F}$ & 4.89423800 & 1.24740400 & 0.33482800 \\
\hline $\mathrm{C}$ & 0.17140100 & 2.99378600 & 0.36957700 \\
\hline $\mathrm{H}$ & -0.78236000 & 3.28243700 & 0.80070400 \\
\hline $\mathrm{H}$ & 0.94818400 & 3.22298500 & 1.09811500 \\
\hline $\mathrm{C}$ & 0.39509600 & 3.70704900 & -0.96600400 \\
\hline $\mathrm{H}$ & 1.33389400 & 3.41296100 & -1.44209000 \\
\hline $\mathrm{H}$ & -0.42846900 & 3.50343700 & -1.65501900 \\
\hline $\mathrm{H}$ & 0.42930100 & 4.78382100 & -0.77753900 \\
\hline $\mathrm{C}$ & -4.91413300 & -0.85842300 & 0.12041800 \\
\hline $\mathrm{H}$ & -4.92330600 & -1.70263700 & 0.80806200 \\
\hline $\mathrm{H}$ & -5.60807500 & -0.08830100 & 0.44794600 \\
\hline $\mathrm{H}$ & -5.19317500 & -1.20317700 & -0.87881000 \\
\hline C & -0.03055700 & -2.68882700 & -0.73288100 \\
\hline $\mathrm{H}$ & -0.96842700 & -2.82706400 & -1.26078300 \\
\hline $\mathrm{H}$ & 0.77561400 & -2.78412100 & -1.46092200 \\
\hline C & 0.09563100 & -3.71348100 & 0.39679500 \\
\hline $\mathrm{H}$ & 0.09160700 & -4.71315000 & -0.04785100 \\
\hline $\mathrm{H}$ & 1.02130100 & -3.59798700 & 0.96616900 \\
\hline $\mathrm{H}$ & -0.75206200 & -3.63606000 & 1.07928000 \\
\hline C & 4.96462300 & -1.54242300 & -0.10813900 \\
\hline $\mathrm{H}$ & 5.60103000 & -1.16103500 & -0.91549200 \\
\hline $\mathrm{H}$ & 5.54171300 & -1.44046600 & 0.81849400 \\
\hline $\mathrm{H}$ & 4.76474200 & -2.59963500 & -0.28578500 \\
\hline
\end{tabular}


Table S6r. Cartesian coordinates for 4B.

\begin{tabular}{|c|c|c|c|}
\hline \multirow[t]{2}{*}{ Atom } & \multicolumn{3}{|c|}{ Coordinates (Angstroms) } \\
\hline & $X$ & $\mathrm{Y}$ & $\mathrm{Z}$ \\
\hline $\mathrm{C}$ & 2.37793300 & -1.45282900 & -0.18110300 \\
\hline $\mathrm{C}$ & 1.20680100 & -0.69851700 & -0.10863500 \\
\hline $\mathrm{C}$ & 1.32318200 & 0.70580300 & 0.12986800 \\
\hline $\mathrm{C}$ & 2.60863900 & 1.28541000 & 0.26532200 \\
\hline $\mathrm{C}$ & -1.04988000 & 0.88170800 & 0.12244700 \\
\hline $\mathrm{C}$ & -1.17341400 & -0.52844900 & -0.06684600 \\
\hline $\mathrm{C}$ & -2.51953700 & -1.10226900 & 0.01014800 \\
\hline $\mathrm{C}$ & -3.39118600 & 1.19028000 & 0.10705100 \\
\hline $\mathrm{H}$ & 2.37465000 & -2.52005500 & -0.34476800 \\
\hline $\mathrm{H}$ & 2.70069400 & 2.34917500 & 0.43063300 \\
\hline $\mathrm{N}$ & -3.57328800 & -0.18472000 & 0.09864600 \\
\hline $\mathrm{N}$ & -2.10102300 & 1.68593200 & 0.23620000 \\
\hline $\mathrm{N}$ & -0.04700800 & -1.29485100 & -0.26849000 \\
\hline $\mathrm{N}$ & 0.17974500 & 1.48259900 & 0.21204800 \\
\hline 0 & -2.76447700 & -2.31007100 & 0.00276100 \\
\hline 0 & -4.33199600 & 1.97946700 & 0.02593500 \\
\hline $\mathrm{C}$ & 3.61683600 & -0.82676900 & -0.02964800 \\
\hline $\mathrm{C}$ & 3.78460300 & 0.54381000 & 0.19360500 \\
\hline $\mathrm{C}$ & 0.26271100 & 2.96511600 & 0.35812800 \\
\hline $\mathrm{H}$ & -0.68241200 & 3.28234000 & 0.78783900 \\
\hline $\mathrm{H}$ & 1.04515900 & 3.17481600 & 1.08616900 \\
\hline $\mathrm{C}$ & 0.50614500 & 3.66733500 & -0.97986900 \\
\hline $\mathrm{H}$ & 1.43637300 & 3.34548800 & -1.45474000 \\
\hline $\mathrm{H}$ & -0.32259600 & 3.48362200 & -1.66834300 \\
\hline $\mathrm{H}$ & 0.56969700 & 4.74358300 & -0.79591800 \\
\hline $\mathrm{C}$ & -4.93965400 & -0.72906400 & 0.12316800 \\
\hline $\mathrm{H}$ & -4.98252400 & -1.54905300 & 0.83890300 \\
\hline $\mathrm{H}$ & -5.61419800 & 0.07137600 & 0.41578800 \\
\hline $\mathrm{H}$ & -5.21549200 & -1.10023500 & -0.86721100 \\
\hline $\mathrm{C}$ & -0.11449800 & -2.71404400 & -0.72510000 \\
\hline $\mathrm{H}$ & -1.05448500 & -2.83181100 & -1.25388100 \\
\hline $\mathrm{H}$ & 0.69045500 & -2.84043600 & -1.44978000 \\
\hline $\mathrm{C}$ & -0.01911700 & -3.72589500 & 0.41877400 \\
\hline $\mathrm{H}$ & -0.04595400 & -4.73128700 & -0.01180500 \\
\hline $\mathrm{H}$ & 0.90679900 & -3.62514300 & 0.99070100 \\
\hline $\mathrm{H}$ & -0.86740500 & -3.61775200 & 1.09642500 \\
\hline $\mathrm{C}$ & 5.14822200 & 1.15598400 & 0.34044400 \\
\hline $\mathrm{H}$ & 5.69211200 & 0.69818500 & 1.17297800 \\
\hline $\mathrm{H}$ & 5.74491500 & 0.99729600 & -0.56388300 \\
\hline $\mathrm{H}$ & 5.07192300 & 2.22894600 & 0.52289200 \\
\hline $\mathrm{F}$ & 4.70537300 & -1.60473200 & -0.10854600 \\
\hline
\end{tabular}


Table S6s. Cartesian coordinates for 5A.

\begin{tabular}{|c|c|c|c|}
\hline \multirow[t]{2}{*}{ Atom } & \multicolumn{3}{|c|}{ Coordinates (Angstroms) } \\
\hline & $X$ & $\mathrm{Y}$ & $\mathrm{Z}$ \\
\hline $\mathrm{C}$ & -2.06254200 & -1.58117600 & -0.19432900 \\
\hline C & -0.92120300 & -0.78247500 & -0.12498100 \\
\hline $\mathrm{C}$ & -1.08708800 & 0.61727100 & 0.09901400 \\
\hline $\mathrm{C}$ & -2.39365900 & 1.14204600 & 0.21953100 \\
\hline C & 1.27473500 & 0.88777200 & 0.11086700 \\
\hline C & 1.45333700 & -0.51867700 & -0.06793200 \\
\hline C & 2.82048400 & -1.03728500 & 0.02216300 \\
\hline C & 3.60070400 & 1.28905900 & 0.12651900 \\
\hline $\mathrm{H}$ & -1.97043100 & -2.64591100 & -0.34716300 \\
\hline $\mathrm{H}$ & -2.54948800 & 2.19897000 & 0.37409900 \\
\hline $\mathrm{N}$ & 3.83586900 & -0.07789500 & 0.12094700 \\
\hline $\mathrm{N}$ & 2.28915900 & 1.73344100 & 0.23028600 \\
\hline $\mathrm{N}$ & 0.35874300 & -1.32872600 & -0.27502600 \\
\hline $\mathrm{N}$ & 0.01966800 & 1.44120600 & 0.18216500 \\
\hline O & 3.11569600 & -2.23400700 & 0.01811800 \\
\hline O & 4.51107700 & 2.11456300 & 0.06353600 \\
\hline C & -3.37293500 & -1.07130100 & -0.06582000 \\
\hline C & -3.50418500 & 0.31515500 & 0.14081300 \\
\hline C & -0.12596000 & 2.92051100 & 0.30709200 \\
\hline $\mathrm{H}$ & -0.92574200 & 3.10822100 & 1.02240600 \\
\hline $\mathrm{H}$ & 0.79967400 & 3.28211900 & 0.74430300 \\
\hline C & -0.38062100 & 3.59320100 & -1.04397600 \\
\hline $\mathrm{H}$ & 0.46484000 & 3.43550100 & -1.71834700 \\
\hline $\mathrm{H}$ & -1.28942300 & 3.22512000 & -1.52689100 \\
\hline $\mathrm{H}$ & -0.49283800 & 4.66814300 & -0.87657300 \\
\hline C & 5.22177400 & -0.56963800 & 0.16225000 \\
\hline $\mathrm{H}$ & 5.51516200 & -0.95114400 & -0.81904200 \\
\hline $\mathrm{H}$ & 5.86388800 & 0.26200200 & 0.44005100 \\
\hline $\mathrm{H}$ & 5.29333100 & -1.37226900 & 0.89539700 \\
\hline C & 0.48933000 & -2.74447500 & -0.72836200 \\
\hline $\mathrm{H}$ & -0.30594100 & -2.90780800 & -1.45622900 \\
\hline $\mathrm{H}$ & 1.43602200 & -2.82173700 & -1.25269900 \\
\hline C & 0.43463000 & -3.75857600 & 0.41638200 \\
\hline $\mathrm{H}$ & 0.50895000 & -4.76215500 & -0.01292600 \\
\hline $\mathrm{H}$ & 1.27424400 & -3.61139400 & 1.09733200 \\
\hline $\mathrm{H}$ & -0.49720700 & -3.69914200 & 0.98427100 \\
\hline C & -4.55045800 & -1.98753100 & -0.15261500 \\
\hline $\mathrm{H}$ & -5.14862100 & -1.93428200 & 0.76489100 \\
\hline $\mathrm{H}$ & -5.21371700 & -1.68700900 & -0.97261000 \\
\hline $\mathrm{H}$ & -4.23939800 & -3.02049500 & -0.31187100 \\
\hline $\mathrm{Cl}$ & -5.08468400 & 1.03113900 & 0.30294500 \\
\hline
\end{tabular}

Table S6t. Cartesian coordinates for 5B. 


\begin{tabular}{|c|c|c|c|}
\hline \multirow[t]{2}{*}{ Atom } & \multicolumn{3}{|c|}{ Coordinates (Angstroms) } \\
\hline & $X$ & $\mathrm{Y}$ & $\mathrm{Z}$ \\
\hline $\mathrm{C}$ & -2.24510300 & -1.16262400 & -0.14729500 \\
\hline $\mathrm{C}$ & -1.01309400 & -0.51559600 & -0.08895800 \\
\hline $\mathrm{C}$ & -1.00269200 & 0.89275000 & 0.13351500 \\
\hline $\mathrm{C}$ & -2.23465400 & 1.58044100 & 0.25215400 \\
\hline $\mathrm{C}$ & 1.37820400 & 0.86092800 & 0.11906900 \\
\hline $\mathrm{C}$ & 1.37274300 & -0.55948300 & -0.05931800 \\
\hline $\mathrm{C}$ & 2.66659100 & -1.24570100 & -0.00018900 \\
\hline $\mathrm{C}$ & 3.73431900 & 0.96501700 & 0.08290000 \\
\hline $\mathrm{H}$ & -2.31188900 & -2.23010600 & -0.29138700 \\
\hline $\mathrm{H}$ & -2.23226700 & 2.65103300 & 0.39594500 \\
\hline $\mathrm{N}$ & 3.79506500 & -0.42276700 & 0.06869800 \\
\hline $\mathrm{N}$ & 2.49058100 & 1.57090100 & 0.20066100 \\
\hline $\mathrm{N}$ & 0.18497900 & -1.22567600 & -0.24353800 \\
\hline $\mathrm{N}$ & 0.20060600 & 1.56683500 & 0.21828700 \\
\hline 0 & 2.80708800 & -2.47077400 & -0.00321400 \\
\hline 0 & 4.74343200 & 1.66545300 & 0.01564600 \\
\hline $\mathrm{Cl}$ & -4.92163100 & -1.34226100 & -0.10386200 \\
\hline $\mathrm{C}$ & -3.44873300 & -0.44415400 & -0.01088800 \\
\hline $\mathrm{C}$ & -3.47423100 & 0.94777800 & 0.18632500 \\
\hline $\mathrm{C}$ & 0.25003800 & 3.04703100 & 0.39303500 \\
\hline $\mathrm{H}$ & -0.52585500 & 3.31442500 & 1.10921800 \\
\hline $\mathrm{H}$ & 1.21010700 & 3.26746500 & 0.84998700 \\
\hline $\mathrm{C}$ & 0.10331900 & 3.79905500 & -0.93153200 \\
\hline $\mathrm{H}$ & 0.92609300 & 3.55411900 & -1.60790900 \\
\hline $\mathrm{H}$ & -0.84238200 & 3.57817500 & -1.43334700 \\
\hline $\mathrm{H}$ & 0.13694400 & 4.87223800 & -0.72274100 \\
\hline $\mathrm{C}$ & 5.10669600 & -1.08889900 & 0.08443600 \\
\hline $\mathrm{H}$ & 5.30974100 & -1.54112600 & -0.88936100 \\
\hline $\mathrm{H}$ & 5.86005400 & -0.33820500 & 0.30697400 \\
\hline $\mathrm{H}$ & 5.10386300 & -1.86744400 & 0.84702500 \\
\hline $\mathrm{C}$ & 0.12381800 & -2.64989500 & -0.68488600 \\
\hline $\mathrm{H}$ & -0.69541100 & -2.71392100 & -1.40156900 \\
\hline $\mathrm{H}$ & 1.04516200 & -2.85494700 & -1.22001100 \\
\hline $\mathrm{C}$ & -0.04764000 & -3.63784200 & 0.47093200 \\
\hline $\mathrm{H}$ & -0.11410600 & -4.64599300 & 0.05120400 \\
\hline $\mathrm{H}$ & 0.81452600 & -3.59811100 & 1.13854800 \\
\hline $\mathrm{H}$ & -0.95427300 & -3.45023100 & 1.05208500 \\
\hline $\mathrm{C}$ & -4.75331200 & 1.72431300 & 0.31948900 \\
\hline $\mathrm{H}$ & -5.37246700 & 1.61257500 & -0.57674200 \\
\hline $\mathrm{H}$ & -5.34297500 & 1.36195100 & 1.16798700 \\
\hline $\mathrm{H}$ & -4.54751700 & 2.78533400 & 0.46879200 \\
\hline
\end{tabular}

Table S6u. Cartesian coordinates for 6A. 


\begin{tabular}{|c|c|c|c|}
\hline \multirow[t]{2}{*}{ Atom } & \multicolumn{3}{|c|}{ Coordinates (Angstroms) } \\
\hline & $X$ & $\mathrm{Y}$ & $\mathrm{Z}$ \\
\hline $\mathrm{C}$ & -2.45022200 & -1.34865400 & -0.13248600 \\
\hline $\mathrm{C}$ & -1.22650800 & -0.64615700 & -0.07620200 \\
\hline $\mathrm{C}$ & -1.27070500 & 0.74504800 & 0.12761500 \\
\hline $\mathrm{C}$ & -2.55835600 & 1.37473000 & 0.23438400 \\
\hline $\mathrm{C}$ & 1.11527500 & 0.86059700 & 0.10779700 \\
\hline $\mathrm{C}$ & 1.16626700 & -0.54839600 & -0.06329000 \\
\hline $\mathrm{C}$ & 2.49286600 & -1.16080600 & -0.01368200 \\
\hline $\mathrm{C}$ & 3.43377300 & 1.12560600 & 0.10324200 \\
\hline $\mathrm{H}$ & -2.42764200 & -2.42192700 & -0.26139000 \\
\hline $\mathrm{H}$ & -2.62521100 & 2.44391600 & 0.37249800 \\
\hline $\mathrm{N}$ & 3.56661500 & -0.27029500 & 0.03274200 \\
\hline $\mathrm{N}$ & 2.16637200 & 1.64623600 & 0.18368600 \\
\hline $\mathrm{N}$ & 0.01242200 & -1.28580100 & -0.23015500 \\
\hline $\mathrm{N}$ & -0.13666500 & 1.49498600 & 0.21276700 \\
\hline 0 & 2.70878800 & -2.37927800 & -0.00351100 \\
\hline 0 & 4.42897200 & 1.85641300 & 0.10865700 \\
\hline $\mathrm{C}$ & -3.71461700 & -0.76504500 & -0.02463800 \\
\hline $\mathrm{C}$ & -3.77580500 & 0.63174400 & 0.15685900 \\
\hline $\mathrm{C}$ & 4.90786400 & -0.86736500 & 0.04648100 \\
\hline $\mathrm{H}$ & 5.63106600 & -0.05708200 & 0.04399700 \\
\hline $\mathrm{H}$ & 5.03135300 & -1.48461300 & 0.93923000 \\
\hline $\mathrm{H}$ & 5.03870600 & -1.49747800 & -0.83561700 \\
\hline $\mathrm{C}$ & -0.16431600 & 2.97107500 & 0.40073300 \\
\hline $\mathrm{H}$ & -0.96540800 & 3.20569600 & 1.10013300 \\
\hline $\mathrm{H}$ & 0.77725300 & 3.22473000 & 0.88068200 \\
\hline $\mathrm{C}$ & -0.30559500 & 3.72739500 & -0.92214800 \\
\hline $\mathrm{H}$ & 0.53260900 & 3.50419300 & -1.58658900 \\
\hline $\mathrm{H}$ & -1.23821300 & 3.48270500 & -1.43935100 \\
\hline $\mathrm{H}$ & -0.30216400 & 4.80059700 & -0.71062200 \\
\hline $\mathrm{C}$ & 0.02782900 & -2.69965600 & -0.69002800 \\
\hline $\mathrm{H}$ & -0.78678200 & -2.79800200 & -1.41030300 \\
\hline $\mathrm{H}$ & 0.96024700 & -2.85451400 & -1.22329300 \\
\hline $\mathrm{C}$ & -0.09853500 & -3.71257400 & 0.44999500 \\
\hline $\mathrm{H}$ & 0.75998900 & -3.63722300 & 1.11976300 \\
\hline $\mathrm{H}$ & -1.01358500 & -3.57635200 & 1.03281100 \\
\hline $\mathrm{H}$ & -0.11565600 & -4.71889600 & 0.02044400 \\
\hline $\mathrm{C}$ & -4.97064200 & -1.58672500 & -0.09953700 \\
\hline $\mathrm{H}$ & -5.56711500 & -1.48949600 & 0.81647300 \\
\hline $\mathrm{H}$ & -5.60404600 & -1.27220600 & -0.93916800 \\
\hline $\mathrm{H}$ & -4.73910500 & -2.64431100 & -0.23622500 \\
\hline $\mathrm{N}$ & -4.93854600 & 1.30519000 & 0.26340800 \\
\hline $\mathrm{H}$ & -4.96333600 & 2.30688000 & 0.39361600 \\
\hline $\mathrm{H}$ & -5.82785800 & 0.82919300 & 0.21405000 \\
\hline
\end{tabular}

Table S6v. Cartesian coordinates for 6B. 


\begin{tabular}{|c|c|c|c|}
\hline \multirow[t]{2}{*}{ Atom } & \multicolumn{3}{|c|}{ Coordinates (Angstroms) } \\
\hline & $X$ & $\mathrm{Y}$ & $\mathrm{Z}$ \\
\hline $\mathrm{C}$ & -2.38273600 & -1.43161100 & -0.18673800 \\
\hline $\mathrm{C}$ & -1.20240900 & -0.67315000 & -0.11538100 \\
\hline $\mathrm{C}$ & -1.30924600 & 0.72068500 & 0.11556000 \\
\hline $\mathrm{C}$ & -2.60159000 & 1.29655700 & 0.24047400 \\
\hline $\mathrm{C}$ & 1.07871000 & 0.89418700 & 0.10312800 \\
\hline $\mathrm{C}$ & 1.17207900 & -0.52619100 & -0.07286000 \\
\hline $\mathrm{C}$ & 2.51586200 & -1.11547200 & -0.01666400 \\
\hline $\mathrm{C}$ & 3.40829900 & 1.17599800 & 0.11024600 \\
\hline $\mathrm{H}$ & -2.34693600 & -2.49902400 & -0.34360400 \\
\hline $\mathrm{H}$ & -2.68606700 & 2.36353200 & 0.39272800 \\
\hline $\mathrm{N}$ & 3.57413500 & -0.21250400 & 0.03676100 \\
\hline $\mathrm{N}$ & 2.13056100 & 1.68414700 & 0.16945900 \\
\hline $\mathrm{N}$ & 0.04054200 & -1.28198000 & -0.25310100 \\
\hline $\mathrm{N}$ & -0.16066900 & 1.49633000 & 0.20106100 \\
\hline 0 & 2.74862600 & -2.32949100 & -0.00626500 \\
\hline 0 & 4.38386200 & 1.92974300 & 0.13454700 \\
\hline $\mathrm{C}$ & -3.66440200 & -0.83535400 & -0.03919600 \\
\hline $\mathrm{C}$ & -3.77534400 & 0.57440500 & 0.17479200 \\
\hline $\mathrm{N}$ & -4.75176400 & -1.62381200 & -0.11014400 \\
\hline $\mathrm{H}$ & -4.67364100 & -2.62240700 & -0.25148400 \\
\hline $\mathrm{H}$ & -5.68631400 & -1.24892500 & -0.02315200 \\
\hline $\mathrm{C}$ & 4.92751300 & -0.78525400 & 0.05975300 \\
\hline $\mathrm{H}$ & 5.63547800 & 0.03839900 & 0.05649200 \\
\hline $\mathrm{H}$ & 5.05898400 & -1.39313600 & 0.95753600 \\
\hline $\mathrm{H}$ & 5.07446300 & -1.41594500 & -0.81879100 \\
\hline $\mathrm{C}$ & -0.23656900 & 2.96697000 & 0.38401500 \\
\hline $\mathrm{H}$ & -1.02572700 & 3.16900900 & 1.10857800 \\
\hline $\mathrm{H}$ & 0.70578100 & 3.26784700 & 0.83331600 \\
\hline $\mathrm{C}$ & -0.45971900 & 3.71234200 & -0.93373300 \\
\hline $\mathrm{H}$ & 0.37151300 & 3.53530800 & -1.62141700 \\
\hline $\mathrm{H}$ & -1.38920500 & 3.41315400 & -1.42586100 \\
\hline $\mathrm{H}$ & -0.51285900 & 4.78544700 & -0.72732000 \\
\hline $\mathrm{C}$ & 0.10022800 & -2.71514000 & -0.67870800 \\
\hline $\mathrm{H}$ & -0.70282000 & -2.85602500 & -1.40177500 \\
\hline $\mathrm{H}$ & 1.03993100 & -2.84962500 & -1.20324800 \\
\hline $\mathrm{C}$ & 0.00291100 & -3.69743600 & 0.48942300 \\
\hline $\mathrm{H}$ & 0.85456900 & -3.57708300 & 1.16092000 \\
\hline $\mathrm{H}$ & -0.92033800 & -3.57531200 & 1.06231500 \\
\hline $\mathrm{H}$ & 0.02253400 & -4.71415400 & 0.08529600 \\
\hline $\mathrm{C}$ & -5.12083800 & 1.22783400 & 0.32229100 \\
\hline $\mathrm{H}$ & -5.74320300 & 1.07561400 & -0.56828600 \\
\hline $\mathrm{H}$ & -5.66955500 & 0.82632200 & 1.18330700 \\
\hline $\mathrm{H}$ & -5.01263000 & 2.30350300 & 0.47151600 \\
\hline
\end{tabular}

Table S6w. Cartesian coordinates for 7A. 


\begin{tabular}{|c|c|c|c|}
\hline \multirow[t]{2}{*}{ Atom } & \multicolumn{3}{|c|}{ Coordinates (Angstroms) } \\
\hline & $X$ & $\mathrm{Y}$ & $\mathrm{Z}$ \\
\hline $\mathrm{C}$ & -3.32074900 & -1.28002900 & -0.06939300 \\
\hline $\mathrm{C}$ & -2.00042100 & -1.73329000 & -0.19566300 \\
\hline $\mathrm{C}$ & -0.89237000 & -0.88015100 & -0.12995800 \\
\hline $\mathrm{C}$ & -1.13014700 & 0.51444300 & 0.07919500 \\
\hline $\mathrm{C}$ & -2.45622900 & 0.98419500 & 0.19817300 \\
\hline $\mathrm{C}$ & -3.53738400 & 0.11018200 & 0.13059600 \\
\hline $\mathrm{C}$ & 1.22210300 & 0.89057300 & 0.08952800 \\
\hline $\mathrm{C}$ & 1.46667400 & -0.50094600 & -0.06850900 \\
\hline $\mathrm{C}$ & 2.85202400 & -0.95655300 & 0.03883100 \\
\hline $\mathrm{C}$ & 3.52462700 & 1.40379800 & 0.13113700 \\
\hline $\mathrm{H}$ & -1.86038300 & -2.79417800 & -0.34096900 \\
\hline $\mathrm{H}$ & -2.63848700 & 2.03661400 & 0.34009300 \\
\hline $\mathrm{N}$ & 3.82212600 & 0.04922400 & 0.14516600 \\
\hline $\mathrm{N}$ & 2.19578300 & 1.78909300 & 0.20587000 \\
\hline $\mathrm{N}$ & 0.40726800 & -1.36623100 & -0.27312400 \\
\hline $\mathrm{N}$ & -0.06027500 & 1.38816500 & 0.14619400 \\
\hline 0 & 3.20373400 & -2.13950800 & 0.04377900 \\
\hline 0 & 4.40200600 & 2.26796300 & 0.07483500 \\
\hline $\mathrm{C}$ & -0.27357500 & 2.86000600 & 0.24137400 \\
\hline $\mathrm{H}$ & -1.07808200 & 3.02639300 & 0.95691100 \\
\hline $\mathrm{H}$ & 0.63533800 & 3.27468800 & 0.66610700 \\
\hline $\mathrm{C}$ & -0.56570100 & 3.49253600 & -1.12145800 \\
\hline $\mathrm{H}$ & 0.28321900 & 3.35872900 & -1.79698300 \\
\hline $\mathrm{H}$ & -1.45853400 & 3.07292300 & -1.59207600 \\
\hline $\mathrm{H}$ & -0.72596800 & 4.56490400 & -0.97745800 \\
\hline $\mathrm{C}$ & 5.22855500 & -0.37565700 & 0.20955200 \\
\hline $\mathrm{H}$ & 5.57114300 & -0.70252700 & -0.77589800 \\
\hline $\mathrm{H}$ & 5.82116000 & 0.47374200 & 0.53934200 \\
\hline $\mathrm{H}$ & 5.31728700 & -1.20290900 & 0.91233700 \\
\hline $\mathrm{C}$ & 0.60713800 & -2.77227800 & -0.72718000 \\
\hline $\mathrm{H}$ & 1.55835000 & -2.80367300 & -1.24849400 \\
\hline $\mathrm{H}$ & -0.17720300 & -2.97423500 & -1.45743200 \\
\hline $\mathrm{C}$ & 0.59894200 & -3.79158500 & 0.41440600 \\
\hline $\mathrm{H}$ & -0.33606200 & -3.77908400 & 0.98019300 \\
\hline $\mathrm{H}$ & 0.72323400 & -4.78961800 & -0.01665300 \\
\hline $\mathrm{H}$ & 1.42886400 & -3.60504100 & 1.09756500 \\
\hline 0 & -4.82330200 & 0.48505300 & 0.24333400 \\
\hline $\mathrm{C}$ & -5.14461900 & 1.87083400 & 0.45689700 \\
\hline $\mathrm{H}$ & -4.80651400 & 2.47929800 & -0.38730000 \\
\hline $\mathrm{H}$ & -6.23026600 & 1.90669600 & 0.52677200 \\
\hline $\mathrm{H}$ & -4.69995600 & 2.22952300 & 1.39011400 \\
\hline $\mathrm{C}$ & -4.47517000 & -2.22474700 & -0.14385400 \\
\hline $\mathrm{H}$ & -5.07175300 & -2.17611100 & 0.77521900 \\
\hline $\mathrm{H}$ & -5.15112900 & -1.94255900 & -0.96022400 \\
\hline $\mathrm{H}$ & -4.14026000 & -3.25138800 & -0.29754700 \\
\hline
\end{tabular}


Table S6x. Cartesian coordinates for 7B.

\begin{tabular}{|c|c|c|c|}
\hline \multirow[t]{2}{*}{ Atom } & \multicolumn{3}{|c|}{ Coordinates (Angstroms) } \\
\hline & $X$ & $\mathrm{Y}$ & $\mathrm{Z}$ \\
\hline C & 3.49420000 & -0.25835400 & -0.01047300 \\
\hline $\mathrm{C}$ & 2.31108400 & -1.02235700 & 0.12145700 \\
\hline $\mathrm{C}$ & 1.05055000 & -0.41635600 & 0.07461900 \\
\hline C & 0.97845500 & 0.98850400 & -0.13225800 \\
\hline $\mathrm{C}$ & 2.18583700 & 1.72978400 & -0.24292500 \\
\hline C & 3.43897500 & 1.15238600 & -0.18849900 \\
\hline $\mathrm{C}$ & -1.40414300 & 0.85824000 & -0.11040700 \\
\hline $\mathrm{C}$ & -1.33047200 & -0.55964600 & 0.05899300 \\
\hline C & -2.59452700 & -1.30247500 & 0.01231900 \\
\hline C & -3.75819800 & 0.86106100 & -0.09305600 \\
\hline $\mathrm{H}$ & 2.39870700 & -2.08839600 & 0.25146000 \\
\hline $\mathrm{H}$ & 2.13628600 & 2.80186700 & -0.36965300 \\
\hline $\mathrm{N}$ & -3.75677000 & -0.53148100 & -0.02684100 \\
\hline $\mathrm{N}$ & -2.54574900 & 1.52025800 & -0.17432100 \\
\hline $\mathrm{N}$ & -0.11297100 & -1.17723100 & 0.22321600 \\
\hline $\mathrm{N}$ & -0.25320100 & 1.61269900 & -0.20795000 \\
\hline 0 & -2.68221600 & -2.53417500 & 0.00177400 \\
\hline 0 & -4.80800200 & 1.50608200 & -0.09382800 \\
\hline 0 & 4.70990800 & -0.79315200 & 0.02918500 \\
\hline C & 4.91096700 & -2.21446400 & 0.19869500 \\
\hline $\mathrm{H}$ & 4.46106100 & -2.76140500 & -0.63336700 \\
\hline $\mathrm{H}$ & 5.99054500 & -2.34655200 & 0.19331700 \\
\hline $\mathrm{H}$ & 4.49677500 & -2.54383700 & 1.15468300 \\
\hline C & -0.36371800 & 3.08664400 & -0.38023600 \\
\hline $\mathrm{H}$ & 0.39992300 & 3.38972100 & -1.09600900 \\
\hline $\mathrm{H}$ & -1.33230300 & 3.26897200 & -0.83650000 \\
\hline C & -0.24779100 & 3.84294100 & 0.94482200 \\
\hline $\mathrm{H}$ & -1.05836200 & 3.56150200 & 1.62202600 \\
\hline $\mathrm{H}$ & 0.70651400 & 3.65950400 & 1.44580000 \\
\hline $\mathrm{H}$ & -0.32612400 & 4.91504000 & 0.74161600 \\
\hline C & -5.03316500 & -1.26206400 & -0.02721600 \\
\hline $\mathrm{H}$ & -5.09481000 & -1.89339300 & 0.86089400 \\
\hline $\mathrm{H}$ & -5.83496700 & -0.52938300 & -0.02532800 \\
\hline $\mathrm{H}$ & -5.09839600 & -1.89243800 & -0.91624100 \\
\hline $\mathrm{C}$ & 0.00652300 & -2.60356000 & 0.64852800 \\
\hline $\mathrm{H}$ & -0.90117700 & -2.84952700 & 1.18952900 \\
\hline $\mathrm{H}$ & 0.83306200 & -2.64593200 & 1.35801000 \\
\hline C & 0.20309100 & -3.57233000 & -0.51920900 \\
\hline $\mathrm{H}$ & 1.09468300 & -3.34241700 & -1.10869100 \\
\hline $\mathrm{H}$ & 0.31393200 & -4.58188300 & -0.11203800 \\
\hline $\mathrm{H}$ & -0.66753200 & -3.55949500 & -1.17681400 \\
\hline C & 4.70268800 & 1.95416200 & -0.30862900 \\
\hline $\mathrm{H}$ & 5.32809100 & 1.83316500 & 0.58238200 \\
\hline $\mathrm{H}$ & 5.29831700 & 1.61807300 & -1.16429000 \\
\hline $\mathrm{H}$ & 4.47695300 & 3.01436200 & -0.43572400 \\
\hline
\end{tabular}


Table S6y. Cartesian coordinates for 8A.

\begin{tabular}{|c|c|c|c|}
\hline \multirow[t]{2}{*}{ Atom } & \multicolumn{3}{|c|}{ Coordinates (Angstroms) } \\
\hline & $\mathrm{X}$ & $\mathrm{Y}$ & $\mathrm{Z}$ \\
\hline $\mathrm{C}$ & -1.46431800 & 1.72844900 & -0.00124000 \\
\hline $\mathrm{C}$ & -0.36504900 & 0.87107000 & -0.00463900 \\
\hline $\mathrm{C}$ & -0.59477300 & -0.51251700 & -0.25513000 \\
\hline $\mathrm{C}$ & -1.92536000 & -0.96298300 & -0.41589400 \\
\hline $\mathrm{C}$ & 1.74779800 & -0.91548100 & -0.14503500 \\
\hline $\mathrm{C}$ & 1.99120900 & 0.47751500 & 0.06933900 \\
\hline $\mathrm{C}$ & 3.38849000 & 0.91517900 & 0.08430600 \\
\hline $\mathrm{C}$ & 4.04155100 & -1.45219200 & -0.02910800 \\
\hline $\mathrm{H}$ & -1.32074400 & 2.78996200 & 0.13641700 \\
\hline $\mathrm{H}$ & -2.12729700 & -2.01557300 & -0.55200600 \\
\hline $\mathrm{N}$ & 4.35118000 & -0.10000800 & 0.03657200 \\
\hline $\mathrm{N}$ & 2.71394500 & -1.81685700 & -0.20810900 \\
\hline $\mathrm{N}$ & 0.93360600 & 1.34697600 & 0.21405300 \\
\hline $\mathrm{N}$ & 0.46657600 & -1.39397300 & -0.30701400 \\
\hline 0 & 3.75400800 & 2.09234800 & 0.13094600 \\
\hline 0 & 4.90335200 & -2.32747700 & 0.04573800 \\
\hline $\mathrm{N}$ & -4.30323300 & -0.62629200 & -0.58653500 \\
\hline $\mathrm{H}$ & -4.39847500 & -1.32622300 & -1.31218600 \\
\hline $\mathrm{C}$ & -3.01211200 & -0.09164700 & -0.38639600 \\
\hline $\mathrm{C}$ & -2.79413200 & 1.29300500 & -0.21491600 \\
\hline C & -5.34651800 & -0.49904600 & 0.30952000 \\
\hline C & -6.63361500 & -1.18381500 & -0.08900800 \\
\hline $\mathrm{H}$ & -7.02696300 & -1.72396500 & 0.77497200 \\
\hline $\mathrm{H}$ & -7.36361700 & -0.41580100 & -0.36468600 \\
\hline $\mathrm{H}$ & -6.51830600 & -1.87148800 & -0.92935100 \\
\hline 0 & -5.23167000 & 0.14846500 & 1.34868400 \\
\hline $\mathrm{C}$ & 5.76057200 & 0.31733000 & 0.09458300 \\
\hline $\mathrm{H}$ & 6.37612600 & -0.55958300 & -0.08595800 \\
\hline $\mathrm{H}$ & 5.94284000 & 1.07656800 & -0.66608100 \\
\hline $\mathrm{H}$ & 5.98718600 & 0.73468100 & 1.07854100 \\
\hline C & 0.25063500 & -2.85478000 & -0.51205200 \\
\hline $\mathrm{H}$ & -0.53252600 & -2.96307500 & -1.26181200 \\
\hline $\mathrm{H}$ & 1.17143800 & -3.24197900 & -0.93793400 \\
\hline $\mathrm{C}$ & -0.08522200 & -3.58609100 & 0.78995000 \\
\hline $\mathrm{H}$ & 0.74064800 & -3.50628800 & 1.50133800 \\
\hline $\mathrm{H}$ & -0.99352000 & -3.20137600 & 1.26096800 \\
\hline $\mathrm{H}$ & -0.24095700 & -4.64402600 & 0.55988000 \\
\hline $\mathrm{C}$ & 1.11456100 & 2.75564200 & 0.66994400 \\
\hline $\mathrm{H}$ & 2.02494200 & 2.78317700 & 1.25977300 \\
\hline $\mathrm{H}$ & 0.28097600 & 2.97269000 & 1.33782400 \\
\hline C & 1.20046900 & 3.75991200 & -0.48167600 \\
\hline $\mathrm{H}$ & 1.29757200 & 4.76271300 & -0.05497900 \\
\hline $\mathrm{H}$ & 2.07920500 & 3.56032100 & -1.09667800 \\
\hline $\mathrm{H}$ & 0.31150200 & 3.74458100 & -1.11781400 \\
\hline
\end{tabular}




$\begin{array}{lllr}\mathrm{C} & -3.88883700 & 2.30554800 & -0.29341600 \\ \mathrm{H} & -4.63225700 & 2.01873800 & -1.04313800 \\ \mathrm{H} & -4.41754100 & 2.36571300 & 0.66622800 \\ \mathrm{H} & -3.49236700 & 3.29402700 & -0.53384000\end{array}$

Table S6z. Cartesian coordinates for 8B.

\begin{tabular}{|c|c|c|c|}
\hline \multirow[t]{2}{*}{ Atom } & \multicolumn{3}{|c|}{ Coordinates (Angstroms) } \\
\hline & $X$ & $\mathrm{Y}$ & $\mathrm{Z}$ \\
\hline $\mathrm{C}$ & 1.98123300 & -0.51651900 & 0.07613300 \\
\hline $\mathrm{C}$ & 0.64582100 & -0.10890400 & 0.03932300 \\
\hline $\mathrm{C}$ & 0.35551900 & 1.26524000 & -0.15237000 \\
\hline $\mathrm{C}$ & 1.43593000 & 2.17805500 & -0.26995100 \\
\hline $\mathrm{C}$ & -1.97998700 & 0.77496500 & -0.09848500 \\
\hline C & -1.68401300 & -0.62034300 & 0.04727500 \\
\hline $\mathrm{C}$ & -2.82187600 & -1.54713400 & 0.00604600 \\
\hline C & -4.30292800 & 0.41662000 & -0.04104300 \\
\hline $\mathrm{H}$ & 2.25240400 & -1.55079600 & 0.19991800 \\
\hline $\mathrm{H}$ & 1.22296100 & 3.22996000 & -0.39427400 \\
\hline $\mathrm{N}$ & -4.08737700 & -0.96166100 & 0.00300200 \\
\hline $\mathrm{N}$ & -3.20710100 & 1.25329800 & -0.13032700 \\
\hline $\mathrm{N}$ & -0.38636600 & -1.04502200 & 0.18420500 \\
\hline $\mathrm{N}$ & -0.95668300 & 1.69678500 & -0.20848900 \\
\hline 0 & -2.72107500 & -2.77755000 & -0.03009500 \\
\hline 0 & -5.44004800 & 0.88981600 & -0.01612100 \\
\hline C & 2.76509300 & 1.80041500 & -0.22724100 \\
\hline $\mathrm{C}$ & 3.04582000 & 0.41389800 & -0.05509200 \\
\hline $\mathrm{C}$ & -5.23760600 & -1.87796500 & 0.01081100 \\
\hline $\mathrm{H}$ & -6.14023700 & -1.27675400 & 0.07043400 \\
\hline $\mathrm{H}$ & -5.24210300 & -2.47652100 & -0.90256900 \\
\hline $\mathrm{H}$ & -5.16818600 & -2.54484100 & 0.87169900 \\
\hline $\mathrm{C}$ & -1.29239200 & 3.13692700 & -0.36656600 \\
\hline $\mathrm{H}$ & -0.59678200 & 3.55760300 & -1.09267300 \\
\hline $\mathrm{H}$ & -2.28546400 & 3.17161000 & -0.80548300 \\
\hline C & -1.26928600 & 3.89426400 & 0.96265100 \\
\hline $\mathrm{H}$ & -2.01103900 & 3.48394000 & 1.65280300 \\
\hline $\mathrm{H}$ & -0.28746400 & 3.85863100 & 1.44257000 \\
\hline $\mathrm{H}$ & -1.51836200 & 4.94238300 & 0.77244600 \\
\hline C & -0.03889500 & -2.44834100 & 0.55821100 \\
\hline $\mathrm{H}$ & -0.88877700 & -2.85211000 & 1.09755900 \\
\hline $\mathrm{H}$ & 0.79613100 & -2.38610100 & 1.25552600 \\
\hline $\mathrm{C}$ & 0.28861200 & -3.33048400 & -0.64827900 \\
\hline $\mathrm{H}$ & 0.55007600 & -4.32816700 & -0.28284200 \\
\hline $\mathrm{H}$ & -0.57959500 & -3.41977500 & -1.30327500 \\
\hline $\mathrm{H}$ & 1.13318900 & -2.94711500 & -1.22666800 \\
\hline $\mathrm{C}$ & 3.86207300 & 2.82363800 & -0.35703000 \\
\hline $\mathrm{H}$ & 4.50435300 & 2.84499300 & 0.53191600 \\
\hline $\mathrm{H}$ & 4.49732400 & 2.62938200 & -1.22979800 \\
\hline
\end{tabular}




$\begin{array}{lrrr}\mathrm{H} & 3.43860200 & 3.82184400 & -0.47776900 \\ \mathrm{~N} & 4.35847300 & 0.00636000 & -0.01153300 \\ \mathrm{H} & 5.04504300 & 0.74682900 & -0.10298600 \\ \mathrm{C} & 4.90849000 & -1.28062700 & 0.14907500 \\ \mathrm{O} & 4.21115200 & -2.27614200 & 0.27314100 \\ \mathrm{C} & 6.41541500 & -1.29716000 & 0.15897100 \\ \mathrm{H} & 6.79600200 & -0.74242300 & 1.02351500 \\ \mathrm{H} & 6.75434200 & -2.33056600 & 0.21638900 \\ \mathrm{H} & 6.81858700 & -0.82857400 & -0.74447400\end{array}$

Table S6aa. Cartesian coordinates for 9A.

\begin{tabular}{|c|c|c|c|}
\hline \multirow[t]{2}{*}{ Atom } & \multicolumn{3}{|c|}{ Coordinates (Angstroms) } \\
\hline & $X$ & $\mathrm{Y}$ & $\mathrm{Z}$ \\
\hline $\mathrm{C}$ & 3.29476300 & 1.33589100 & -0.08061800 \\
\hline $\mathrm{C}$ & 1.95598100 & 1.77658600 & -0.19940100 \\
\hline $\mathrm{C}$ & 0.86375500 & 0.91043800 & -0.13331100 \\
\hline $\mathrm{C}$ & 1.12319600 & -0.47200600 & 0.07009500 \\
\hline $\mathrm{C}$ & 2.46918800 & -0.90921700 & 0.18011500 \\
\hline $\mathrm{C}$ & 3.55618300 & -0.04296600 & 0.11324500 \\
\hline $\mathrm{C}$ & -1.21956500 & -0.89954900 & 0.08718200 \\
\hline $\mathrm{C}$ & -1.48746500 & 0.49638200 & -0.06673600 \\
\hline $\mathrm{C}$ & -2.88450100 & 0.92295700 & 0.03254200 \\
\hline $\mathrm{C}$ & -3.51209100 & -1.45177000 & 0.12415200 \\
\hline $\mathrm{H}$ & 1.79987400 & 2.83597200 & -0.34117000 \\
\hline $\mathrm{H}$ & 2.66137000 & -1.96161200 & 0.31980100 \\
\hline $\mathrm{N}$ & -3.83506300 & -0.10010200 & 0.11639200 \\
\hline $\mathrm{N}$ & -2.17398400 & -1.81111000 & 0.18460900 \\
\hline $\mathrm{N}$ & -0.45017200 & 1.37909000 & -0.26454000 \\
\hline $\mathrm{N}$ & 0.07450400 & -1.36884100 & 0.14654600 \\
\hline 0 & -3.25841000 & 2.09888300 & 0.04967500 \\
\hline 0 & -4.37429700 & -2.33038500 & 0.09715200 \\
\hline $\mathrm{C}$ & 0.31543100 & -2.83535600 & 0.25702300 \\
\hline $\mathrm{H}$ & 1.12539900 & -2.98016500 & 0.97094200 \\
\hline $\mathrm{H}$ & -0.58485600 & -3.26075000 & 0.68979900 \\
\hline $\mathrm{C}$ & 0.61332400 & -3.47845000 & -1.09941800 \\
\hline $\mathrm{H}$ & -0.23692500 & -3.36081500 & -1.77630900 \\
\hline $\mathrm{H}$ & 1.50161500 & -3.05370500 & -1.57435700 \\
\hline $\mathrm{H}$ & 0.78557400 & -4.54755900 & -0.94517100 \\
\hline $\mathrm{C}$ & -5.24675200 & 0.30891000 & 0.17190400 \\
\hline $\mathrm{H}$ & -5.84583600 & -0.58239600 & 0.33595000 \\
\hline $\mathrm{H}$ & -5.38625000 & 1.01879200 & 0.98774900 \\
\hline $\mathrm{H}$ & -5.53491500 & 0.78435000 & -0.76847200 \\
\hline $\mathrm{C}$ & -0.67297000 & 2.79062500 & -0.69012800 \\
\hline $\mathrm{H}$ & 0.10620200 & 3.02015500 & -1.41738800 \\
\hline $\mathrm{H}$ & -1.62562600 & 2.81873100 & -1.20888800 \\
\hline $\mathrm{C}$ & -0.67776600 & 3.78377800 & 0.47413200 \\
\hline $\mathrm{H}$ & 0.25733600 & 3.76893800 & 1.03984000 \\
\hline
\end{tabular}




$\begin{array}{rrrr}\mathrm{H} & -0.81411900 & 4.78991800 & 0.06630500 \\ \mathrm{H} & -1.50549200 & 3.57133800 & 1.15243300 \\ \mathrm{C} & 5.17050300 & -2.03924300 & 0.46231700 \\ \mathrm{H} & 6.23792600 & -2.26286500 & 0.54127200 \\ \mathrm{H} & 4.69344500 & -2.37873900 & 1.38783300 \\ \mathrm{H} & 4.77140200 & -2.62967800 & -0.36905400 \\ \mathrm{C} & 4.40001300 & 2.34101100 & -0.17110600 \\ \mathrm{H} & 5.01519900 & 2.32962500 & 0.73733800 \\ \mathrm{H} & 5.07641700 & 2.10328000 & -1.00186000 \\ \mathrm{H} & 4.01569700 & 3.35141100 & -0.31633400 \\ \mathrm{C} & 4.98122900 & -0.53705600 & 0.24022700 \\ \mathrm{H} & 5.45831300 & 0.01170000 & 1.06301100 \\ \mathrm{H} & 5.52818800 & -0.23294500 & -0.66222900 \\ ---------------------------------------------------\end{array}$

Table S6ab. Cartesian coordinates for 9B.

\begin{tabular}{|c|c|c|c|}
\hline \multirow[t]{2}{*}{ Atom } & \multicolumn{3}{|c|}{ Coordinates (Angstroms) } \\
\hline & $X$ & $\mathrm{Y}$ & Z \\
\hline $\mathrm{C}$ & -3.47834500 & -0.35595200 & -0.24174900 \\
\hline $\mathrm{C}$ & -2.25754300 & -1.06987600 & -0.34177900 \\
\hline $\mathrm{C}$ & -1.00837900 & -0.46203100 & -0.22259200 \\
\hline $\mathrm{C}$ & -0.97044000 & 0.93483900 & 0.03951300 \\
\hline $\mathrm{C}$ & -2.19490300 & 1.64785100 & 0.11968800 \\
\hline $\mathrm{C}$ & -3.44076900 & 1.03833700 & -0.01484400 \\
\hline $\mathrm{C}$ & 1.40776000 & 0.84546200 & 0.13208500 \\
\hline $\mathrm{C}$ & 1.37530000 & -0.56576200 & -0.10440400 \\
\hline $\mathrm{C}$ & 2.65042600 & -1.28234000 & -0.03221400 \\
\hline $\mathrm{C}$ & 3.76287400 & 0.89525700 & 0.23119200 \\
\hline $\mathrm{H}$ & -2.32701000 & -2.13414200 & -0.51612900 \\
\hline $\mathrm{H}$ & -2.17217000 & 2.71503300 & 0.28803900 \\
\hline $\mathrm{N}$ & 3.79363500 & -0.49000900 & 0.11619900 \\
\hline $\mathrm{N}$ & 2.52891600 & 1.52509400 & 0.29808500 \\
\hline $\mathrm{N}$ & 0.17881200 & -1.19582200 & -0.35557300 \\
\hline $\mathrm{N}$ & 0.24121800 & 1.57733500 & 0.19993800 \\
\hline 0 & 2.76990000 & -2.50933300 & -0.08998000 \\
\hline 0 & 4.79137600 & 1.56922300 & 0.28997000 \\
\hline $\mathrm{C}$ & 0.31515700 & 3.04777800 & 0.43040200 \\
\hline $\mathrm{H}$ & -0.48974900 & 3.30861700 & 1.11640400 \\
\hline $\mathrm{H}$ & 1.25612800 & 3.22976600 & 0.94107200 \\
\hline $\mathrm{C}$ & 0.25041600 & 3.84899400 & -0.87206800 \\
\hline $\mathrm{H}$ & 1.10069000 & 3.60952600 & -1.51566800 \\
\hline $\mathrm{H}$ & -0.67328200 & 3.66463400 & -1.42687100 \\
\hline $\mathrm{H}$ & 0.29459000 & 4.91374200 & -0.62529200 \\
\hline $\mathrm{C}$ & 5.08553200 & -1.19269600 & 0.14408400 \\
\hline $\mathrm{H}$ & 5.86271800 & -0.45338500 & 0.31521700 \\
\hline $\mathrm{H}$ & 5.08067700 & -1.93351000 & 0.94483800 \\
\hline $\mathrm{H}$ & 5.25255700 & -1.69910100 & -0.80887900 \\
\hline $\mathrm{C}$ & 0.10222900 & -2.60387100 & -0.84003100 \\
\hline
\end{tabular}




$\begin{array}{lrrr}\mathrm{H} & -0.69945500 & -2.63320900 & -1.57804200 \\ \mathrm{H} & 1.03353400 & -2.81308200 & -1.35611400 \\ \mathrm{C} & -0.11870600 & -3.61990500 & 0.28270200 \\ \mathrm{H} & -1.03927300 & -3.43107400 & 0.84115600 \\ \mathrm{H} & -0.18862400 & -4.61604900 & -0.16449600 \\ \mathrm{H} & 0.72383800 & -3.61158500 & 0.97600600 \\ \mathrm{C} & -4.76363900 & -1.12391200 & -0.35516900 \\ \mathrm{H} & -5.54385700 & -0.49067800 & -0.78539600 \\ \mathrm{H} & -4.61823800 & -1.97090200 & -1.03396600 \\ \mathrm{C} & -5.24644600 & -1.66547000 & 1.01554500 \\ \mathrm{H} & -4.50846800 & -2.34445100 & 1.45239200 \\ \mathrm{H} & -5.42646200 & -0.85209700 & 1.72412700 \\ \mathrm{H} & -6.18146300 & -2.21573900 & 0.87669200 \\ \mathrm{C} & -4.69542800 & 1.86844400 & 0.07151200 \\ \mathrm{H} & -5.24132900 & 1.85481300 & -0.87847000 \\ \mathrm{H} & -5.37413900 & 1.48926600 & 0.84177900 \\ \mathrm{H} & -4.46006500 & 2.90741300 & 0.30989000 \\ -------13000\end{array}$

